\title{
TENSOR STRUCTURES ARISING FROM AFFINE LIE ALGEBRAS. II
}

\author{
D. KAZHDAN AND G. LUSZTIG
}

\section{INTRODUCTION}

In Part I of this paper we studied a category $\mathscr{O}_{\kappa}$ of representations of an affine Lie algebra with central charge $\kappa-h$ (where $\kappa \in \mathbf{C}-\mathbf{Q}_{\geq 0}$ ) and we defined a tensor product functor which associates to several objects of $\mathscr{O}_{\kappa}$ another object of $\mathscr{O}_{\kappa}$. Here we will establish the commutativity isomorphism and the associativity isomorphism for this tensor product functor.

The numbering of sections will continue that of Part $I$.

In $\S 9$ we consider certain modules of coinvariants and prove some finiteness results for them. (The idea to consider such spaces of coinvariants has been used in the case of $\widetilde{\mathscr{O}}_{\kappa}$ in the work of Beilinson and Feigin cited in the introduction to Part I.) In $\S \S 10-12$ we introduce integrable connections on these modules of coinvariants; these should be regarded as generalizations of the Knizhnik-Zamolodchikov equations. In $\S 14$ we establish the commutativity isomorphisms, and in $\S \S 15-18$ we establish the associativity isomorphisms.

We are very indebted to A. Beilinson for many useful discussions. The idea to use formal schemes in the definition of the associativity isomorphism was suggested to us by him; the definition of the diagonal modules (see 16.10), which plays a crucial role in the proof, is due to A. Beilinson.

We also thank Lepowsky and Huang for useful discussions.

We have benefited from reading Deligne's manuscript [De], which contains a discussion of the connection between tensor categories and local systems on the moduli space of stable marked curves of genus 0 .

\section{CONTENTS}

9. Finiteness of coinvariants

10. Vector fields

11. Sugawara operators and coinvariants

12. Connections

13. Tensor product and coinvariants

14. Commutativity isomorphism

15. Degeneration of quadrics (I)

16. Degeneration of quadrics (II)

17. Degeneration of quadrics and connections

Received by the editors October 5, 1992.

1991 Mathematics Subject Classification. Primary 20G99.

Both authors are supported in part by the National Science Foundation. 
18. The associativity isomorphism

Appendix: Induced modules

\section{FINITENESS OF COINVARIANTS}

To $n$ objects in $\mathscr{O}_{\kappa}$ and to $n$ distinct points of $P^{1}$ with given charts, one can associate a finite-dimensional vector space, using coinvariants in the usual tensor product with respect to an action of the Lie algebra $\mathbf{g}$ with coefficients regular functions on $P^{1}$ minus the given points. When the $n$ points and the charts are allowed to vary in all possible ways, we thus obtain a vector bundle over the space of parameters.

9.1. We shall denote $H=P G L_{2}(\mathbf{C})$. Any element $\gamma \in H$, represented by a matrix $\left(\begin{array}{ll}r & s \\ t & u\end{array}\right)$, defines an automorphism $z \mapsto \gamma(z)=\frac{r z+s}{t z+u}$ of the projective line $P^{1}=\mathrm{C} \cup\{\infty\}$.

We fix a finite set $S$ with $|S| \geq 2$.

We consider the commutative diagram

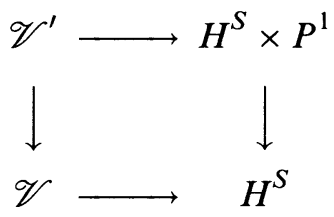

where $\mathscr{V}$ is the the affine open subset of $H^{S}$ consisting of all $\left(\gamma_{s}\right)$ such that $\gamma_{s}(0) \quad(s \in S)$ are $|S|$ distinct points of $P^{1}, \mathscr{V}^{\prime}$ is the affine open subset of $H^{S} \times P^{1}$ consisting of all $\left(\left(\gamma_{s}\right), z\right)$ such that $z$ and $\gamma_{s}(0) \quad(s \in S)$ are $|S|+1$ distinct points of $P^{1}$, the horizontal maps are the obvious open imbeddings, and the vertical maps are given by $\left(\left(\gamma_{s}\right), z\right) \mapsto\left(\gamma_{s}\right)$.

9.2. Consider the free action of $H$ on $H^{S}$ given by

$$
\left.\gamma:\left(\gamma_{s}\right)_{s \in S} \rightarrow\left(\gamma \gamma_{s}\right)_{s \in S}\right)
$$

and the free action of $H$ on $H^{S} \times P^{1}$ given by

$$
\gamma:\left(\left(\gamma_{s}\right), z\right) \mapsto\left(\left(\gamma \gamma_{s}\right), \gamma(z)\right) .
$$

The open subsets $\mathscr{V}, \mathscr{V}^{\prime}$ of $H^{S}, H^{S} \times P^{1}$ are $H$-stable; hence, by restriction we have free actions of $H$ on $\mathscr{V}, \mathscr{V}^{\prime}$. The orbit spaces form again a commutative diagram

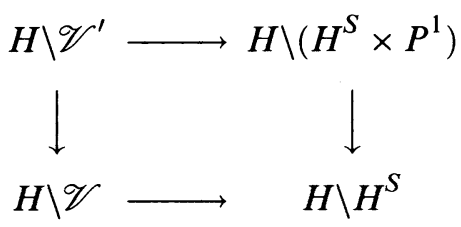

We denote $\underline{\mathscr{V}}=H \backslash \mathscr{V}$ and $\underline{\mathscr{V}^{\prime}}=H \backslash \mathscr{V}^{\prime}$. Then $\underline{\mathscr{V}}$ is an affine open subset of $H \backslash H^{S}$ and $\underline{\mathscr{V}^{\prime}}$ is an affine open subset of $H \backslash\left(H^{S} \times P^{1}\right)$. 
9.3. Generally, for any complex algebraic variety $X$, we shall denote by $\mathbf{C}[X]$ the $\mathbf{C}$-algebra of all regular functions $X \rightarrow \mathbf{C}$.

Let $A=\mathbf{C}[\mathscr{V}], A^{\prime}=\mathbf{C}\left[\mathscr{V}^{\prime}\right]$. The surjective map $\underline{\mathscr{V}^{\prime}} \rightarrow \underline{\mathscr{V}}$ in the diagram in 9.2 induces an imbedding of algebras $A \subset A^{\prime}$; we shall thus regard $A$ as a subalgebra of $A^{\prime}$.

9.4. Let $s \in S$ and let $f \in A^{\prime}$. We consider $u \in \mathscr{V}$; we represent it by a point $\widetilde{u}=\left(\gamma_{t}\right)_{t \in S}$ in $\mathscr{V}$ (so that $u$ is the $H$-orbit of $\left.\widetilde{u}\right)$. Then $z \mapsto\left(\widetilde{u}, \gamma_{s}(z)\right.$ ) is a well-defined regular function from a nonempty Zariski open set in $P^{1}$ to $\mathscr{V}^{\prime}$ (independent of the choice of $\widetilde{u}$ ). The composition of this function with $f: \mathscr{V}^{\prime} \rightarrow \mathbf{C}$ is then a rational function $P^{1} \rightarrow P^{1}$. The power series expansion of this rational function at 0 is denoted $\sum_{n} p_{n, s}(u) \epsilon^{n} \in \mathbf{C}((\epsilon))$.

Then $u \mapsto p_{n, s}(u)$ is an element $p_{n, s}$ of $A$. It satisfies $p_{n, s}=0$ for $n \ll 0$.

We set

$$
{ }^{s} f=\sum_{n} p_{n, s} \epsilon^{n} \in A((\epsilon)) .
$$

Then $f \mapsto^{s} f$ is a homomorphism of $A$-algebras $A^{\prime} \rightarrow A((\epsilon))$.

9.5. For any $s \in S$, we define a function $f_{s} \in A^{\prime}$ by the following requirement: the value of $f_{s}$ at the $H$-orbit of $\left(\left(\gamma_{t}\right)_{t \in S}, z\right) \in \mathscr{V}^{\prime}$ is equal to

$$
\frac{1}{\gamma_{s}^{-1}(z)} \in \mathbf{C}
$$

From the definition we have

$$
{ }^{s} f_{s}=\epsilon^{-1} \text { and } \quad{ }^{s} f_{s^{\prime}} \in A[[\epsilon]] \quad \text { for } s^{\prime} \neq s .
$$

Lemma 9.6. The functions

$$
f_{s}^{k} \quad(s \in S ; k \geq 1) \text { and } 1
$$

form a basis of $A^{\prime}$ as an A-module.

Let $f \in A^{\prime}$. For any $s \in S$ we can write ${ }^{s} f=\sum_{n<0} a_{s, n} \epsilon^{n}+g_{s}$ where $g_{s} \in A[[\epsilon]]$ and $a_{s, n} \in A$ are zero for $n \ll 0$. Let $r=\sum_{n<0 ; s} a_{s, n} f_{s}^{-n} \in A^{\prime}$.

Since ${ }^{s} f_{s}=\epsilon^{-1}$ and ${ }^{s^{\prime}} f_{s} \in A[[\epsilon]] \quad \forall s^{\prime} \neq s$, we have

$$
{ }^{s}(f-r)=g_{s}-\sum_{s^{\prime}: s^{\prime} \neq s} \sum_{n<0} a_{s^{\prime}, n}\left({ }^{s} f_{s^{\prime}}\right)^{-n} \in A[[\epsilon]] .
$$

Thus the function $f-r$ when restricted to any fibre of $\mathscr{V}^{\prime} \rightarrow \underline{\mathscr{V}}$ (a projective line with some missing points) has no poles at those missing points and hence is constant. It follows that $f-r \in A$. This shows that the functions (a) span $A^{\prime}$ as an $A$-module.

We show that they are linearly independent.

Assume that $a_{0}+\sum_{s} \sum_{n>0} a_{s, n} f_{s}^{n}=0$ in $A^{\prime}$ where $a_{0}, a_{s, n} \in A$ and all but finitely many of them are zero. Our assumption implies for any $s$ :

$$
0={ }^{s} 0=a_{0}+\sum_{n>0} a_{s, n} \epsilon^{-n}+\mu_{s}
$$


where $\mu_{s} \in A[[\epsilon]]$ (see 9.5(b)). It follows that $a_{s, n}=0$ for all $s$ and all $n>0$. Introducing this in our assumption we get also $a_{0}=0$. The lemma is proved.

9.7. Assume that we are given a commutative $\mathbf{C}$-algebra $B$ and a homomorphism of C-algebras $A \rightarrow B$.

Tensoring the map $A^{\prime} \rightarrow A((\epsilon)) \quad\left(f \mapsto{ }^{s} f\right)$ with $B$ over $A$ we obtain a homomorphism of $B$-algebras $B^{\prime} \rightarrow B \otimes_{A} A((\epsilon))$, where $B^{\prime}=B \otimes_{A} A^{\prime}$. Composing it with the obvious $B$-algebra homomorphism $B \otimes_{A} A((\epsilon)) \rightarrow B((\epsilon))$, we obtain a $B$-algebra homomorphism $B^{\prime} \rightarrow B((\epsilon))$ which will be denoted again by $f \mapsto{ }^{s} f$. These maps form together a $B$-algebra homomorphism

$$
B^{\prime} \rightarrow B((\epsilon))^{S}
$$

Recall that $f_{s} \in A^{\prime}$ is defined in 9.5. We shall denote the element $1 \otimes f_{s} \in B^{\prime}$ again by $f_{s}$.

Lemma 9.8. We have an exact sequence of $B$-modules

$$
0 \longrightarrow B \stackrel{\alpha}{\longrightarrow} B^{\prime} \oplus B[[\epsilon]]^{S} \stackrel{\alpha^{\prime}}{\longrightarrow} B((\epsilon))^{S} \rightarrow 0
$$

where $\alpha$ has as components the obvious homomorphisms and $\alpha^{\prime}$ has as second component the obvious imbedding and its first component is minus the map 9.7(a).

Consider an element $\psi=\left(\psi_{s}\right)_{s \in S} \in B((\epsilon))^{S}$. We have $\psi_{s}=\sum_{n} p_{s, n} \epsilon^{n}$ where $p_{s, n} \in B$ is zero for $n \ll 0 \quad(s \in S)$. We write $\psi_{s}=\psi_{s}^{\prime}+\psi_{s}^{\prime \prime}$ where $\psi_{s}^{\prime}=\sum_{n<0} p_{s, n} \epsilon^{n}$ and $\psi_{s}^{\prime \prime} \in B[[\epsilon]]$.

Let $f=\sum_{s^{\prime} \in S} \sum_{n<0} p_{s^{\prime}, n} f_{s^{\prime}}^{-n} \in B^{\prime}$. For any $s \in S$, we have

$$
{ }^{s} f=\sum_{s^{\prime} \in S} \sum_{n<0} p_{s^{\prime}, n}\left({ }^{s}\left(f_{s^{\prime}}\right)\right)^{-n} \in B((\epsilon)) .
$$

The terms with $s^{\prime} \neq s$ are in $B[[\epsilon]]$ (see 9.5(b)); the remaining terms contribute $\sum_{n<0} p_{s, n} \epsilon^{n}=\psi_{s}^{\prime}$. Thus $\left({ }^{s} f\right)-\psi \in B[[\epsilon]]^{S}$. This shows that the map $\alpha^{\prime}$ is surjective.

It is clear that $\alpha^{\prime} \alpha=0$.

Now let $f \in B^{\prime}$ be such that ${ }^{s} f \in B[[\epsilon]]$ for all $s$. Using Lemma 9.6 (or its consequence over $B$ ) we can write uniquely $f=b_{0}+\sum_{s} \sum_{n>0} b_{s, n} f_{s}^{n}$ in $B^{\prime}$ where $b_{0}, b_{s, n} \in B$ and all but finitely many of them are zero.

Our assumption implies for any $s$ that $b_{0}+\sum_{n>0} b_{s, n} \epsilon^{-n} \in B[[\epsilon]]$. It follows that $b_{s, n}=0$ for all $s$ and all $n>0$. Thus, we have $f=b_{0} \in B$. The lemma is proved.

9.9. Let $\Delta=A^{\prime} \otimes \mathbf{g}$. We shall regard $\Delta$ as an $A$-Lie algebra with

$$
\left[f c, f^{\prime} c^{\prime}\right]=f f^{\prime}\left[c, c^{\prime}\right]
$$

for all $f, f^{\prime} \in A^{\prime}$ and all $c, c^{\prime} \in \mathbf{g}$. (We write $f c$ instead of $f \otimes c$.)

We have a natural homomorphism of $A$-Lie algebras

$$
\Delta \rightarrow \widehat{\mathbf{g}}_{A},
$$

given by $f c \mapsto \sum_{s \in S} \delta_{s}\left({ }^{s} f c\right)$. 
The fact that (a) is a Lie algebra homomorphism follows from the residue theorem for differential forms on $P^{1}$.

Let $\Delta_{B}$ be the $B$-Lie algebra $B \otimes_{A} \Delta=B^{\prime} \otimes \mathbf{g}$.

Tensoring (a) with $B$ we obtain a $B$-Lie algebra homomorphism $\Delta_{B} \rightarrow$ $B \otimes_{A} \widehat{\mathbf{g}}_{A}^{S}$. Composing this with the obvious $B$-Lie algebra homomorphism $B \otimes_{A}$ $\widehat{\mathbf{g}}_{A}^{S} \rightarrow \widehat{\mathbf{g}}_{B}^{S}$, we obtain a homomorphism of $B$-Lie algebras

$$
\Delta_{B} \rightarrow \widehat{\mathbf{g}}_{B}^{S}
$$

9.10. Tensoring the exact sequence $9.8(\mathrm{a})$ with $1_{\mathrm{g}}$ we deduce that we have an exact sequence of $B$-modules

$$
0 \rightarrow B \otimes \mathbf{g} \stackrel{\alpha}{\longrightarrow} \Delta_{B} \oplus\left(\widehat{\mathbf{g}}_{B}^{S}\right)^{+} \stackrel{\alpha^{\prime}}{\longrightarrow} \widetilde{\mathbf{g}}_{B}^{S} \rightarrow 0
$$

where $\alpha$ has as components the obvious Lie algebra homomorphisms and $\alpha^{\prime}$ has as second component the obvious imbedding and its first component is minus the map 9.9(b). In particular, $\Delta_{B}$ is identified under 9.9(b) with a Lie subalgebra of $\widehat{\mathbf{g}}_{B}^{S}$.

9.11. Assume that for each $s \in S$ we are given a smooth $\widetilde{\mathbf{g}}_{B}$-module $\underline{V}_{s}$ with central charge $\kappa-h$.

Let $Y=\bigotimes_{s \in S} \underline{V}_{S}$ (tensor product over $B$ ). We regard $Y$ as a $\widehat{\mathbf{g}}_{B}^{S}$-module as in 1.13 and, via $9.9(\mathrm{~b})$, as a $\Delta_{B}$-module. We want to study the $B$-module of coinvariants $Y_{\Delta_{B}}=Y / \Delta_{B} Y$.

Proposition 9.12. Assume that for each $s \in S$ there exists $N_{s} \geq 1$ such that $\underline{V}_{s}\left(N_{s}\right)$ generates $\underline{V}_{s}$ as a $\widetilde{\mathbf{g}}_{B}$-module. Then

(a) $Y$ is the sum of $\Delta_{B} Y$ and of the image of $\bigotimes_{s \in S} \underline{V}_{s}\left(N_{s}\right)$ in $Y$.

(b) If we assume in addition that $\underline{V}_{s}\left(N_{s}\right)$ is a finitely generated B-module, then the B-module of coinvariants $Y_{\Delta_{B}}$ is finitely generated.

Clearly (b) follows from (a). We now prove (a). We have

$$
\underline{V}_{s}=\sum_{\tau \geq 0} Q_{\tau}^{\sharp} \underline{V}_{s}\left(N_{s}\right) \quad \text { for all } s \in S .
$$

It is then enough to verify the following statement.

For any $\tau_{s} \geq 0$, any $\xi_{s} \in Q_{\tau_{s}}^{\sharp}$, and any $y_{s} \in \underline{V}_{s}\left(N_{s}\right)(s \in S)$ we have

$$
\bigotimes_{s \in S}\left(\xi_{s} y_{s}\right) \in \bigotimes_{s \in S} \underline{V}_{s}\left(N_{s}\right)+\Delta_{B} Y
$$

We will prove (a) by induction on $\tau=\sum_{s \in S} \tau_{s}$.

For $\tau=0,(a)$ is trivial. Hence, we may assume that $\tau_{s} \geq 1$ for at least one $s$. We fix such an $s$.

We may assume that $\xi_{s}=\left(\epsilon^{-1} c\right) \xi_{s}^{\prime}$ for some $\xi_{s}^{\prime} \in Q_{\tau_{s}-1}^{\sharp}$ and some $c \in \mathbf{g}$. 
Let $w=\bigotimes_{s^{\prime}}\left(\xi_{s^{\prime}} y_{s^{\prime}}\right)$ and let $w^{\prime}=\bigotimes_{s^{\prime}: s^{\prime} \neq s}\left(\xi_{s^{\prime}} y_{s^{\prime}}\right) \otimes\left(\xi_{s}^{\prime} y_{s}\right)$. By the definition of the $\Delta_{B}$-module structure on $Y$ we have

$$
\left.\left(f_{s} c\right)\left(w^{\prime}\right)=w+\sum_{s_{1} \neq s}\left(\xi_{s}^{\prime}\right) y_{s}\right) \otimes\left(\left({ }^{s_{1}} f_{s} c\right) \xi_{s_{1}} y_{s_{1}}\right) \otimes\left(\bigotimes_{s^{\prime} \neq s, s_{1}}\left(\xi_{s^{\prime}} y_{s^{\prime}}\right)\right)
$$

If $s_{1} \neq s$, we have ${ }^{s_{1}} f_{s} \in B[[\epsilon]]$; hence,

$$
\left({ }^{s_{1}} f_{s} c\right)\left(\xi_{s_{1}} y_{s_{1}}\right) \in \sum_{\tau^{\prime}: \tau^{\prime} \leq \tau_{s_{1}}} Q_{\tau^{\prime}}^{\sharp} \underline{V}_{s_{1}}\left(N_{s_{1}}\right)
$$

(see 1.8(c)).

Using the induction hypothesis, it follows that

$$
w^{\prime}\left(s_{1}\right) \in \bigotimes_{s^{\prime}} \underline{V}_{s^{\prime}}\left(N_{s^{\prime}}\right)+\Delta_{B} Y \text {. }
$$

From (c) and (b) we see that $w \in \bigotimes_{s^{\prime} \in S}{\underline{V_{s^{\prime}}}}\left(N_{s^{\prime}}\right)+\Delta_{B} Y$ and the induction step is established.

9.13. The module of coinvariants $Y / \Delta_{B} Y$ behaves in a very simple way under base change. Namely, if $B \rightarrow B_{1}$ is a homomorphism of $B$ into another commutative $\mathbf{C}$-algebra $B_{1}$ with 1 , then we can form the $\widehat{\mathbf{g}}_{B_{1}}^{S}$-module $Y_{1}=$ $B_{1} \otimes_{B} Y$ analogous to $Y$ and the $B_{1}$-module of coinvariants $Y_{1} / \Delta_{B_{1}} Y_{1}$. We have a natural isomorphism of $B_{1}$-modules

$$
B_{1} \otimes_{B}\left(Y / \Delta_{B} Y\right) \cong Y_{1} / \Delta_{B_{1}} Y_{1} .
$$

Indeed, we have an exact sequence of $B$-modules

$$
\Delta \otimes Y \rightarrow Y \rightarrow Y / \Delta_{B} Y \rightarrow 0
$$

Its tensor product with $B_{1}$ over $B$ is again an exact sequence, and it maps naturally to the exact sequence analogous to (b):

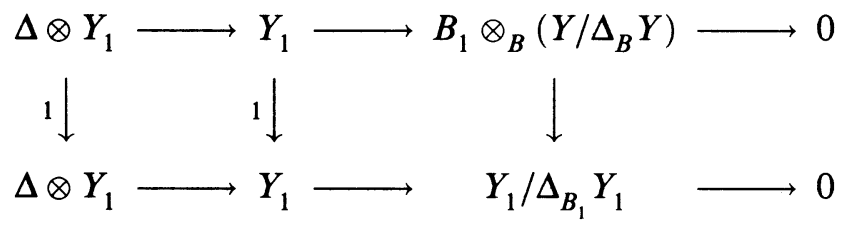

This clearly implies (a).

9.14. Assume now that, for each $s$, we have $\mathscr{V}_{s}=B \otimes V_{s}$ where $V_{s}$ is the generalized Weyl module in $\mathscr{O}_{\kappa}$ corresponding to the nil-module $\mathscr{N}_{S}$ of $\mathbf{C}[\epsilon] \otimes \mathbf{g}$. We shall show that in this case, the space of coinvariants $Y_{\Delta_{B}}=Y / \Delta_{B} Y$ is particularly simple.

Proposition 9.15. In the setup above, the canonical homomorphism

$$
\left(B \otimes\left(\bigotimes_{s} \mathscr{N}_{s}\right)\right)_{\mathrm{g}} \rightarrow Y_{\Delta_{B}}
$$


induced on coinvariants by the obvious inclusion

$$
B \otimes\left(\bigotimes_{s} \mathscr{N}_{s}\right) \subset Y
$$

is an isomorphism. Moreover, we have

$$
\left(B \otimes\left(\bigotimes_{s} \mathscr{N}_{s}\right)\right)_{\mathrm{g}}=B \otimes\left(\left(\bigotimes_{s} \mathscr{N}_{s}\right)_{\mathrm{g}}\right) .
$$

The last assertion is obvious. We now prove the first assertion. We will assume in the proof that $B$ is a finitely generated $\mathbf{C}$-algebra. (Using 9.13, one can reduce the general case to the case where $B=A$, which is certainly a finitely generated $\mathbf{C}$-algebra.)

We can regard $\mathscr{N}_{s}$ as a $\widetilde{\mathbf{g}}^{+}$-module with $\mathbf{1}$ acting as multiplication by $\kappa-$ $h$. Then $\left(\widetilde{\mathbf{g}}, \mathbf{C}\left[\epsilon^{-1}\right] \epsilon^{-1} \otimes \mathbf{g}, \widetilde{\mathbf{g}}^{+}, \mathscr{N}_{s}\right)$ form a split induction datum (see A.1), and with the notation of A.1 we have $V_{s}=\mathscr{N}_{s}^{b}$. It follows easily that $\left(\widetilde{\mathbf{g}}_{B}^{S},\left(B\left[\epsilon^{-1}\right] \epsilon^{-1} \otimes \mathbf{g}\right)^{S},\left(\widetilde{\mathbf{g}}_{B}^{S}\right)^{+}, B \otimes\left(\otimes_{s} \mathscr{N}_{s}\right)\right)$ is a split induction datum and $Y=B \otimes\left(\otimes V_{s}\right)=\left(B \otimes\left(\otimes_{S} \mathscr{N}_{s}\right)\right)^{b}$. This is clearly a smooth $\widetilde{\mathbf{g}}_{B}^{S}$-module; hence, it extends naturally to a $\widehat{\mathbf{g}}_{B}^{S}$-module. From the definition of a split induction datum, it then follows that $\left(\widehat{\mathbf{g}}_{B}^{S},\left(B\left[\epsilon^{-1}\right] \epsilon^{-1} \otimes \mathbf{g}\right)^{S},\left(\widehat{\mathbf{g}}_{B}^{S}\right)^{+}, B \otimes\left(\otimes_{S} \mathscr{N}_{s}\right)\right.$ is a split induction datum with $Y=B \otimes\left(\otimes V_{s}\right)=\left(B \otimes\left(\otimes_{s} \mathscr{N}_{s}\right)\right)^{b}$. (Note that the $\left(\widetilde{\mathbf{g}}_{B}^{S}\right)^{+}$-module $B \otimes\left(\bigotimes_{S} \mathscr{N}_{S}\right)$ extends naturally to a $\left(\widehat{\mathbf{g}}_{B}^{S}\right)^{+}$-module.)

In particular, using A.2, we have that

$$
Y=U\left(\widehat{\mathbf{g}}_{B}^{S}\right) \otimes_{\left.U\left(\widehat{\mathbf{g}}_{B}^{S}\right)^{+}\right)}\left(B \otimes\left(\bigotimes_{s} \mathscr{N}_{s}\right)\right)
$$

as $\widehat{\mathbf{g}}_{B}$-modules.

We denote $\mathbf{h}=\widehat{\mathbf{g}}_{B}^{S}$. We consider its sub-Lie algebras $\mathbf{h}^{+}=\left(\widehat{\mathbf{g}}_{B}^{S}\right)^{+}, \mathbf{h}^{\prime}=\Delta_{B}$, and $\mathbf{h}^{\prime+}=B \otimes \mathbf{g}$. From 9.10 it follows that $\mathbf{h}=\mathbf{h}^{\prime}+\mathbf{h}^{+}$and $\mathbf{h}^{\prime+}=\mathbf{h}^{\prime} \cap \mathbf{h}^{+}$. Using now A.7(a), we see that (a) is surjective.

Assume that the proposition is known whenever $B$ is a finite-dimensional commutative $\mathbf{C}$-algebra. We shall deduce the general case from this as follows. We only have to show that (a) is injective. Let $y$ be an element in the kernel of (a). If we tensor (a) over $B$ with any finite-dimensional quotient algebra $B_{1}$ of $B$, we obtain the map analogous to (a), for $B_{1}$ instead of $B$. (We use the fact that coinvariants behave well under base change; see 9.14.) By our assumption this new map is injective. Since the image of $y$ in $\left(B_{1} \otimes\left(\bigotimes_{s} \mathscr{N}_{s}\right)\right)_{\mathrm{g}}$ is in the kernel of the map (a) (for $B_{1}$ ), it follows that this image is zero. We now use the following known statement: if $E$ is a finite-dimensional vector space over $C$ and $y \in B \otimes E$ has zero image in $B_{1} \otimes E$ for any finite-dimensional quotient algebra $B_{1}$ of $B$, then $y=0$. (We may assume that $E=C$ and we use Krull's theorem .)

We are therefore reduced to the case where $B$ is finite dimensional over $\mathbf{C}$. By 9.10, we can find a $B$-basis of $\mathbf{h}^{\prime}=\Delta_{B}$ containing a $B$-basis of $\mathbf{h}^{\prime+}=B \otimes \mathbf{g}$; moreover, since $B$ is finite dimensional, we have $\left(\widehat{\mathbf{g}}_{B}^{S}\right)^{+}=B \otimes\left(\widehat{\mathbf{g}}^{S}\right)^{+}$, and hence, 
we can find a $B$-basis of $\mathbf{h}^{+}=\left(\widehat{\mathbf{g}}_{B}^{S}\right)^{+}$containing the $B$-basis of $\mathbf{h}^{\prime+}=B \otimes \mathbf{g}$ considered above. These bases form together a basis of $\mathbf{h}$ to which A.7(b) is applicable. We thus obtain the required isomorphism (a).

Proposition 9.16. Assume that for each $s \in S$ we have $\mathscr{V}_{s}=B \otimes V_{s}$ where $V_{s}$ is an object in $\mathscr{O}_{\kappa}$. Then $Y / \Delta_{B} Y$ is a finitely generated $B$-module.

By 2.22, for each $s$ we can find a surjective homomorphism of $\widetilde{\mathbf{g}}$-modules $V_{s}^{\prime} \rightarrow V_{s}$, where $V_{s}^{\prime}$ is a generalized Weyl module. Let $Y^{\prime}$ be the $\Delta_{B}$-module defined in terms of the $V_{s}^{\prime}$ in the same way as $Y$ was defined in terms of the $V_{s}$. The maps $V_{s}^{\prime} \rightarrow V_{s}$ give rise to a surjective map of $\Delta_{B}$-modules $Y^{\prime} \rightarrow Y$, and this induces a surjective map of $B$-modules $Y^{\prime} / \Delta_{B} Y^{\prime} \rightarrow Y / \Delta_{B} Y$. By 9.15 , $Y^{\prime} / \Delta_{B} Y^{\prime}$ is a finitely generated $B$-module, hence so is $Y / \Delta_{B} Y$. (Alternatively, we could have used 9.12 instead of 9.15.)

9.17. Assume now that $|S| \geq 3$ and that we are given an element $s_{0} \in S$; let $S^{\prime}=S-\left\{s_{0}\right\}$. Let $A \rightarrow B=\mathrm{C}$ be the algebra homomorphism given by evaluation at a point $\left(\gamma_{s}\right)_{s \in S}$ of $\underline{\mathscr{V}}$. Let $V_{s}$ be objects of $\mathscr{O}_{\kappa}$ indexed by $S$. We assume that $V_{s_{0}}=\mathbf{V}_{0}^{\kappa}$. Then $\Delta_{\mathbf{C}} \subset \widehat{\mathbf{g}}^{S}$ and $Y / \Delta_{\mathbf{C}} Y$ are defined as before. (Here, $Y=\bigotimes_{s \in S} V_{s}$.)

Replacing now $S$ by $S^{\prime},\left(\gamma_{s}\right)_{s \in S}$ by $\left(\gamma_{s}\right)_{s \in S^{\prime}}$, and $\left(V_{s}\right)_{s \in S}$ by $\left(V_{s}\right)_{s \in S^{\prime}}$, we obtain similarly a Lie subalgebra $\Delta_{\mathbf{C}}^{\prime} \subset \widehat{\mathbf{g}} S^{\prime}$ and a space of coinvariants $Y^{\prime} / \Delta_{\mathbf{C}}^{\prime} Y^{\prime}$. (Here, $Y^{\prime}=\bigotimes_{s \in S^{\prime}} V_{s}$.)

We have $Y=\mathbf{V}_{0}^{\kappa} \otimes Y^{\prime}$, and the natural imbedding $\mathbf{C}=\mathscr{V}_{0} \subset \mathbf{V}_{0}^{\kappa}$ gives by tensoring with $1_{Y^{\prime}}$ an imbedding $Y^{\prime}=\mathbf{C} \otimes Y^{\prime} \subset \mathbf{V}_{0}^{\kappa} \otimes Y^{\prime}=Y$.

Proposition 9.18. The imbedding $Y^{\prime} \subset Y$ described above satisfies $\Delta_{\mathbf{C}}^{\prime} Y^{\prime} \subset \Delta_{\mathbf{C}} Y$ and induces an isomorphism on coinvariants: $Y_{\Delta_{\mathrm{c}}^{\prime}}^{\prime} \cong Y_{\Delta_{\mathrm{c}}}$.

Let $\mathbf{h}=\widehat{\mathbf{g}}^{S}$. Let $\mathbf{h}^{+}$be the Lie subalgebra of $\mathbf{h}$ consisting of all elements $\left(\xi_{s}\right)$ such that $\xi_{s_{0}} \in \widehat{\mathbf{g}}^{+}$. Let $\mathbf{h}^{\prime}=\Delta_{\mathbf{C}}$, regarded as a Lie subalgebra of $\mathbf{h}$. Let $\mathbf{h}^{\prime+}=\Delta_{\mathbf{C}}^{\prime}$, regarded naturally as a Lie subalgebra of $\mathbf{h}^{\prime}$. It is clear from the definitions that $\mathbf{h}^{\prime+}=\mathbf{h}^{\prime} \cap \mathbf{h}^{+}$. From 9.10(a) it follows that $\mathbf{h}=\mathbf{h}^{\prime}+\mathbf{h}^{+}$.

We shall regard $Y^{\prime}$ as an $\mathbf{h}^{+}$-module as follows. Let $\xi \in \widehat{\mathbf{g}}$. If $s \neq s_{0}$, then $\delta_{s} \xi$ acts on $Y^{\prime}$ as in the $\widehat{\mathbf{g}}^{S^{\prime}}$-module structure. If $s=s_{0}$, so that $\xi=\xi^{\prime}+d \mathbf{1}$ with $\xi^{\prime} \in \mathbf{C}((\epsilon)) \otimes \mathbf{g}$ and $d \in \mathbf{C}$, then $\delta_{s} \xi$ acts on $Y^{\prime}$ as multiplication by $d(\kappa-h)$. It follows easily from the definitions that $U(\mathbf{h}) \otimes_{U\left(\mathbf{h}^{+}\right)} Y^{\prime}=Y$. We may apply Proposition A.7(b) to these Lie algebras and to the $\mathbf{h}^{+}$-module $Y^{\prime}$; the proposition follows.

\section{VECTOR FIELDS}

In this section we study the vector fields on the manifold $\underline{\mathscr{V}}$ (as in 9.2).

10.1. A vector field on a smooth affine variety $X$ over $\mathbf{C}$ is an element of the Lie algebra Der $\mathbf{C}[X]$ of derivations of the $\mathbf{C}$-algebra $\mathbf{C}[X]$ (notation of 9.3). 
10.2. We have $\mathbf{C}\left[G L_{2}(\mathbf{C})\right]=\mathbf{C}\left[g_{11}, g_{12}, g_{21}, g_{22},\left(g_{11} g_{22}-g_{12} g_{21}\right)^{-1}\right]$ where $g_{i j}$ are the entries of a matrix $\left(\begin{array}{ll}g_{11} & g_{12} \\ g_{21} & g_{22}\end{array}\right)$.

Recall that $H=P G L_{2}(\mathbf{C})$. Let $A_{0}=\mathbf{C}[H]$. We will identify $A_{0}$ with the subalgebra of the coordinate algebra $\mathbf{C}\left[G L_{2}(\mathbf{C})\right]$ consisting of invariants for the $\mathbf{C}^{*}$ action given by $g_{i j} \mapsto \lambda g_{i j}$ for all $\lambda \in \mathbf{C}^{*}$.

For $i, j \in\{1,2\}$ we consider the vector field $\theta^{i j}=g_{1 i} \partial / \partial g_{1 j}+$ $g_{2 i} \partial / \partial g_{2 j}$ on $G L_{2}(\mathbf{C})$.

We then have

$$
\frac{\partial}{\partial g_{i j}}=\frac{g_{3-i, 3-i} \theta^{i j}-g_{3-i, i} \theta^{3-i, j}}{g_{11} g_{22}-g_{12} g_{21}} .
$$

While the vector fields $\partial / \partial g_{i j}$ do not preserve the subalgebra $A_{0}$, the vector fields $\theta^{i j}$ do and can therefore be regarded as vector fields on $H$. We use the following notation for these vector fields on $H: \theta^{i j}=(-1)^{i-1} \theta_{i-j}$ or equivalently

$$
\theta^{12}=\theta_{-1}, \quad \theta^{11}=-\theta^{22}=\theta_{0}, \quad \theta^{21}=-\theta_{1} .
$$

Note that $\theta_{1}, \theta_{0}, \theta_{-1}$ form an $A_{0}$-basis of the $A_{0}$-module Der $A_{0}$ and a C-basis of the Lie subalgebra of Der $A_{0}$ consisting of derivations of $A_{0}$ which commute with the automorphisms of $A_{0}$ defined by the various left translations in $H$.

10.3. Let $\phi: H \times P^{1} \rightarrow P^{1}$ be the map $\phi(\gamma, z)=\gamma(z)$. To $\phi$ we may apply the vector field $\theta_{k}$ with respect to the variable $\gamma$ or the vector field $\frac{\partial}{\partial z}$ with respect to the variable $z$. A simple computation shows that

$$
\theta_{k}(\phi)=z^{k+1} \frac{\partial \phi}{\partial z}
$$

for $k=-1,0,1$.

Let $S$ and let

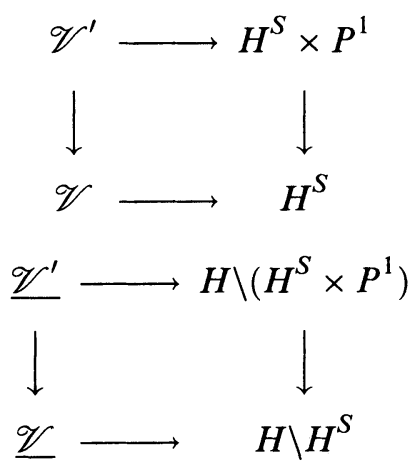

be as in 9.1, 9.2. Recall the notation $A=\mathbf{C}[\underline{\mathscr{V}}], A^{\prime}=\mathbf{C}\left[\mathscr{V}^{\prime}\right]$. We define also $\widetilde{A}=\mathbf{C}[\mathscr{V}], \widetilde{A}^{\prime}=\mathbf{C}\left[\mathscr{V}^{\prime}\right]$. We have $\widetilde{A} \subset \widetilde{A}^{\prime}$ and $A=\widetilde{A}^{H} \subset \widetilde{A}^{\prime H}=A^{\prime}$.

10.4. Given $k \in\{1,0,-1\}$ and $s \in S$, let $\theta_{k, s}$ be the vector field on $H^{S}$ which is $\theta_{k}$ in the $s$-direction and is zero in the directions $s^{\prime}$ for all $s^{\prime} \neq s$. 
Since $\mathscr{V}$ is an open subset of $H^{S}$, we may regard $\theta_{k, s}$ as a vector field on $\mathscr{V}$.

It is easy to check that

$$
\begin{gathered}
{\left[\theta_{0, s}, \theta_{-1, s^{\prime}}\right]=\delta_{s s^{\prime}} \theta_{-1, s}, \quad\left[\theta_{0, s}, \theta_{1, s^{\prime}}\right]=-\delta_{s s^{\prime}} \theta_{1, s},} \\
{\left[\theta_{1, s}, \theta_{-1, s^{\prime}}\right]=2 \delta_{s s^{\prime}} \theta_{0, s^{\prime}} .}
\end{gathered}
$$

Hence the C-subspace $\mathscr{D}_{0}$ of $\operatorname{Der} \tilde{A}$ spanned by the elements $\theta_{k, s}$ for $k \in\{1,0,-1\}$ and $s \in S$ is a Lie subalgebra.

10.5. Let $\mathscr{D}_{1}$ be the set of all derivations of the algebra $\tilde{A}$ which commute with the $H$-action on $\tilde{A}$. It is clear that $\mathscr{D}_{1}$ is a Lie subalgebra and an $A$ submodule of Der $\widetilde{A}$ and that any derivation in $\mathscr{D}_{1}$ maps the subalgebra $A$ into itself, so that we have a natural Lie algebra homomorphism $\mathscr{D}_{1} \rightarrow \operatorname{Der} A$.

Lemma 10.6. (a) The vector fields $\left\{\theta_{k, s} \mid k \in\{1,0,-1\}, s \in S\right\}$ form a C-basis of $\mathscr{D}_{0}$, an $\tilde{A}$-basis of the $\tilde{A}$-module $\operatorname{Der} \tilde{A}$ and an A-basis of the A-module $\mathscr{D}_{1}$.

(b) The Lie algebra homomorphism $\mathscr{D}_{1} \rightarrow \operatorname{Der}(A)$ considered above is surjective.

The proof is standard.

10.7. From the previous lemma we see that $\theta_{k, s} \in \mathscr{D}_{1}$; hence, it may be regarded as a derivation of the algebra $A$.

The vector field $\theta_{k, s}$ on $H^{S}$ may be regarded as a vector field on $H^{S} \times P^{1}$ (zero in the direction of $P^{1}$ ), and this restricts to a vector field on the open set $\mathscr{V}^{\prime}$ or to a derivation of $\widetilde{A}^{\prime}$; it is clear that this maps the subalgebra $A^{\prime}$ of $\widetilde{A}^{\prime}$ into itself and hence defines a derivation of $A^{\prime}$ denoted again $\theta_{k, s}: A^{\prime} \rightarrow A^{\prime}$.

Lemma 10.8. Let $f \in A^{\prime}$, and let $s, s^{\prime} \in S$. Write

$$
s^{\prime} f=\sum_{n} p_{n, s^{\prime}} \epsilon^{n}, \quad s^{\prime}\left(\theta_{k, s} f\right)=\sum_{n} q_{n, s^{\prime}} \epsilon^{n}
$$

where $p_{n, s^{\prime}}, q_{n, s^{\prime}} \in A$. (See 9.4.) Then

$$
q_{n, s^{\prime}}+\delta_{s s^{\prime}}(n-k) p_{n-k, s^{\prime}}=\theta_{k, s}\left(p_{n, s^{\prime}}\right)
$$

for all $n$. (Here $\theta_{k, s} f \in A^{\prime}$ and $\theta_{k, s}\left(p_{n, s^{\prime}}\right) \in A$ are defined as in 10.7.)

We regard $f$ as a function on the open subset $\mathscr{V}^{\prime}$ of $H^{S} \times P^{1}$. Applying $\frac{\partial}{\partial z}$ to $f$, regarded as a function of its last argument $z \in P^{1}$, we obtain an element $\frac{\partial f}{\partial z}$ in the quotient field of $\mathbf{C}\left[\mathscr{V}^{\prime}\right]=\widetilde{A}^{\prime}$.

By definition, we have power series expansions at $y=0$

$$
\begin{gathered}
f\left(\left(\gamma_{t}\right), \gamma_{s^{\prime}}(y)\right)=\sum_{n} p_{n, s^{\prime}} y^{n}, \\
\left(\theta_{k, s} f\right)\left(\left(\gamma_{t}\right), \gamma_{s^{\prime}}(y)\right)=\sum_{n} q_{n, s^{\prime}} y^{n} .
\end{gathered}
$$


We apply the operator $\frac{\partial}{\partial y}$ to (a); we obtain

$$
\frac{\partial f}{\partial z}\left(\left(\gamma_{t}\right), \gamma_{s^{\prime}}(y)\right) \frac{\partial \phi}{\partial y}\left(\gamma_{s^{\prime}}, y\right)=\sum_{n} n p_{n, s^{\prime}} y^{n-1}
$$

where $\phi(\gamma, y)=\gamma(y)$.

Recall from 10.3(a) that $\theta_{k}(\phi)=y^{k+1} \frac{\partial \phi}{\partial y}$. Hence, if we apply the operator $\theta_{k}$ to (a) (with respect to the $s$-coordinate $\gamma_{s}$ ), the left-hand side becomes

$$
\left(\theta_{k, s} f\right)\left(\left(\gamma_{t}\right), \gamma_{s^{\prime}}(y)\right)+\delta_{s s^{\prime}} \frac{\partial f}{\partial z}\left(\left(\gamma_{t}\right), \gamma_{s^{\prime}}(y)\right) y^{k+1} \frac{\partial \phi}{\partial y}\left(\gamma_{s^{\prime}}, y\right)
$$

while the right-hand side becomes $\sum_{n} \theta_{k, s}\left(p_{n, s^{\prime}}\right) y^{n}$. From the equality of these two expressions, we see using (b) and (c) that

$$
\sum_{n} q_{n, s^{\prime}} y^{n}+\delta_{s s^{\prime}} \sum_{n} n p_{n, s^{\prime}} y^{n+k}=\sum_{n} \theta_{k, s}\left(p_{n, s^{\prime}}\right) y^{n} .
$$

We now compare the coefficient of $y^{n}$ in the two sides of the last equality. The lemma follows.

Lemma 10.9. For any $s \in S$ and $k=1,0,-1$, we have

$$
\theta_{k, s} f_{s}=f_{s}^{1-k}
$$

where $f_{s} \in A^{\prime}$ is as in 9.5. Moreover, $\theta_{k, s} f_{s^{\prime}}=0$ for $s \neq s^{\prime}$.

We apply the previous lemma with $f=f_{s^{\prime}}$. By 9.5(b), we have $p_{n, s^{\prime}}=$ $\delta_{n,-1}$ and hence $\theta_{k, s}\left(p_{n, s}\right)=0$. By the previous lemma, we have $q_{n, s^{\prime}}+$ $\delta_{s s^{\prime}}(n-k) \delta_{n-k,-1}=0$; hence, $q_{n, s^{\prime}}=\delta_{s s^{\prime}} \delta_{n-k,-1}$. In other words, ${ }^{s^{\prime}}\left(\theta_{k, s} f_{s^{\prime}}\right)=$ $\delta_{s s^{\prime}} \epsilon^{k-1}$. We have also ${ }^{s^{\prime}}\left(\delta_{s s^{\prime}} f_{s^{\prime}}^{1-k}\right)=\delta_{s s^{\prime}} \epsilon^{k-1}$. Thus, ${ }^{s^{\prime}}\left(\theta_{k, s} f_{s^{\prime}}-\delta_{s s^{\prime}} f_{s^{\prime}}^{1-k}\right)=0$ so that $\theta_{k, s} f_{s^{\prime}}-\delta_{s s^{\prime}} f_{s^{\prime}}^{1-k}=0$, as required.

10.10. Let $s \neq s^{\prime}$ in $S$. According to $9.5(\mathrm{~b})$, we can write

$$
{ }^{s^{\prime}} f_{s}=l_{s s^{\prime}}+m_{s s^{\prime}} \epsilon+\cdots \in A[[\epsilon]], \quad{ }^{s} f_{s^{\prime}}=l_{s^{\prime} s}+m_{s^{\prime} s} \epsilon+\cdots \in A[[\epsilon]]
$$

where $l_{s s^{\prime}}, m_{s s^{\prime}}, l_{s^{\prime} s}, m_{s^{\prime} s}$ are well defined elements of $A$. Let $k_{s s^{\prime}} \in A^{\prime}$ be defined by the equality

$$
f_{s} f_{s^{\prime}}=l_{s s^{\prime}} f_{s^{\prime}}+l_{s^{\prime} s} f_{s}+k_{s s^{\prime}} 1
$$

Clearly, we must have

$$
k_{s s^{\prime}}=k_{s^{\prime} s} .
$$

From the definitions we have

$$
{ }^{s} k_{s s^{\prime}}=\epsilon^{-1}\left(l_{s^{\prime} s}+m_{s^{\prime} s} \epsilon+\cdots\right)-l_{s^{\prime} s} \epsilon^{-1}-l_{s s^{\prime}}\left(l_{s^{\prime} s}+m_{s^{\prime} s} \epsilon+\cdots\right) .
$$

This shows that ${ }^{s} k_{s s^{\prime}} \in A[[\epsilon]]$ and that the coefficient of $\epsilon^{0}$ is $m_{s s^{\prime}}-l_{s s^{\prime}} l_{s^{\prime} s}$. Since $k_{s s^{\prime}}$ is symmetric in $s, s^{\prime}$, we have also that ${ }^{\prime} k_{s s^{\prime}} \in A[[\epsilon]]$ and the coefficient of $\epsilon^{0}$ is $m_{s^{\prime} s}-l_{s s^{\prime}} l_{s^{\prime} s}$. 
If $s^{\prime \prime} \in S$ is different from $s, s^{\prime}$, then $s^{\prime \prime} f_{s}, s^{\prime \prime} f_{s^{\prime}} \in A[[\epsilon]]$ and hence ${ }^{\prime \prime} k_{s s^{\prime}} \in$ $A[[\epsilon]]$.

Thus the function $k_{s s^{\prime}}$ when restricted to any fibre of $\underline{\mathscr{V}}^{\prime} \rightarrow \underline{\mathscr{V}}$ (a projective line with some missing points) has no poles at those missing points and hence is constant. It follows that

$$
k_{s s^{\prime}} \in A \text {. }
$$

We then have ${ }^{s} k_{s s^{\prime}}=k_{s s^{\prime}}$ and similarly ${ }^{s^{\prime}} k_{s s^{\prime}}=k_{s s^{\prime}}$. By the argument above, we then have $k_{s s^{\prime}}=m_{s s^{\prime}}-l_{s s^{\prime}} l_{s^{\prime} s}$ and $k_{s s^{\prime}}=m_{s^{\prime} s}-l_{s s^{\prime}} l_{s^{\prime} s}$. In particular, we have

$$
m_{s s^{\prime}}=m_{s^{\prime} s}=k_{s s^{\prime}}+l_{s s^{\prime}} l_{s^{\prime} s}
$$

Using the definitions, we can write

$$
s^{\prime} f_{s}=\frac{c \epsilon+d}{a \epsilon+b}, \quad{ }^{s} f_{s^{\prime}}=\frac{c^{\prime} \epsilon+d^{\prime}}{a^{\prime} \epsilon+b^{\prime}}
$$

where $\left(\begin{array}{ll}a & b \\ c & d\end{array}\right)\left(\begin{array}{ll}a^{\prime} & b^{\prime} \\ c^{\prime} & d^{\prime}\end{array}\right)=\left(\begin{array}{ll}1 & 0 \\ 0 & 1\end{array}\right)$ (equality of matrices over $A$ ) and $b, b^{\prime}$ are invertible in $A$.

A simple computation shows that

$$
\begin{gathered}
l_{s s^{\prime}}=d b^{-1}, \quad l_{s^{\prime} s}=d^{\prime} b^{\prime-1} \\
k_{s s^{\prime}}=-c b^{-1}=-c^{\prime} b^{\prime-1}, \quad m_{s s^{\prime}}=\left(b b^{\prime}\right)^{-1} .
\end{gathered}
$$

In particular,

(e)

$$
\mathrm{m}_{s s^{\prime}} \text { is an invertible element of } A \text {. }
$$

Lemma 10.11. Let $s \neq s^{\prime}$ in $S$.

(a) We have

$$
\begin{aligned}
& \theta_{1, s}\left(k_{s s^{\prime}}\right)=-l_{s^{\prime} s}, \quad \theta_{0, s}\left(k_{s s^{\prime}}\right)=k_{s s^{\prime}}, \quad \theta_{-1, s}\left(k_{s s^{\prime}}\right)=k_{s s^{\prime}} l_{s s^{\prime}}, \\
& \theta_{1, s}\left(l_{s s^{\prime}}\right)=1, \quad \theta_{0, s}\left(l_{s s^{\prime}}\right)=l_{s s^{\prime}}, \quad \theta_{-1, s}\left(l_{s s^{\prime}}\right)=l_{s s^{\prime}}^{2} \text {, } \\
& \theta_{1, s}\left(l_{s^{\prime} s}\right)=0, \quad \theta_{0, s}\left(l_{s^{\prime} s}\right)=0, \quad \theta_{-1, s}\left(l_{s^{\prime} s}\right)=l_{s s^{\prime}} l_{s^{\prime} s}+k_{s s^{\prime}} \text {, } \\
& \theta_{1, s}\left(m_{s s^{\prime}}\right)=0, \quad \theta_{0, s}\left(m_{s s^{\prime}}\right)=m_{s s^{\prime}}, \quad \theta_{-1, s}\left(m_{s s^{\prime}}\right)=2 l_{s s^{\prime}} m_{s s^{\prime}} \text {. }
\end{aligned}
$$

(b) If $s^{\prime \prime} \in S$ is distinct from $s, s^{\prime}$, then

$$
\theta_{j, s^{\prime \prime}}\left(k_{s s^{\prime}}\right)=\theta_{j, s^{\prime \prime}}\left(l_{s s^{\prime}}\right)=\theta_{j, s^{\prime \prime}}\left(l_{s^{\prime} s}\right)=\theta_{j, s^{\prime \prime}}\left(m_{s s^{\prime}}\right)=0 .
$$

Applying the derivation $\theta_{j, s}$ to $10.10(\mathrm{a})$ (for $j \in\{1,0,-1\}$ ) and using 10.9 , we see that

$$
f_{s}^{1-j} f_{s^{\prime}}=\theta_{j, s}\left(l_{s s^{\prime}}\right) f_{s^{\prime}}+\theta_{j, s}\left(l_{s^{\prime} s}\right) f_{s}+l_{s^{\prime} s} f_{s}^{1-j}+\theta_{j, s}\left(k_{s s^{\prime}}\right) .
$$

Recall that $\theta_{j, s}(A) \subset A$ and that the powers of $f_{s}$ and those of $f_{s^{\prime}}$ are linearly independent over $A$ (see 9.6). Hence if $j=1$, from (a) we deduce the values of $\theta_{1, s}$ applied to $k_{s s^{\prime}}, l_{s s^{\prime}}, l_{s^{\prime} s}$. 
If $j=0$, we replace in (c) $f_{s} f_{s^{\prime}}$ by the expression $10.10(a)$; using again the linear independence in 9.6, we deduce the values of $\theta_{0, s}$ applied to $k_{s s^{\prime}}, l_{s s^{\prime}}$, $l_{s^{\prime} s}$.

If $j=-1$, we replace in (c) $f_{s}^{2} f_{s^{\prime}}$ by the expression

$$
l_{s s^{\prime}}^{2} f_{s^{\prime}}+l_{s s^{\prime}} l_{s^{\prime} s} f_{s^{\prime}}+l_{s^{\prime} s} f_{s}^{2}+k_{s s^{\prime}} f_{s}+l_{s s^{\prime}} k_{s s^{\prime}}
$$

which follows by applying twice $10.10(\mathrm{a})$. Using again the linear independence in 9.6, we deduce the values of $\theta_{-1, s}$ applied to $k_{s s^{\prime}}, l_{s s^{\prime}}, l_{s^{\prime} s}$.

Introducing these values in the identity

$$
\theta_{j, s}\left(m_{s s^{\prime}}\right)=\theta_{j, s}\left(k_{s s^{\prime}}\right)+\theta_{j, s}\left(l_{s s^{\prime}}\right) l_{s^{\prime} s}+l_{s s^{\prime}} \theta_{j, s}\left(l_{s^{\prime} s}\right)
$$

which follows from

$$
m_{s s^{\prime}}=k_{s s^{\prime}}+l_{s s^{\prime}} l_{s^{\prime} s},
$$

we obtain the values of $\theta_{j, s}\left(m_{s s^{\prime}}\right)$. This proves (a).

We now prove (b). Applying the derivation $\theta_{j, s^{\prime \prime}}$ to 10.10 (a) (for $j \in$ $\{1,0,-1\})$ and using 10.9 , we see that

$$
0=\theta_{j, s^{\prime \prime}}\left(l_{s s^{\prime}}\right) f_{s^{\prime}}+\theta_{j, s^{\prime \prime}}\left(l_{s^{\prime} s}\right) f_{s}+\theta_{j, s^{\prime \prime}}\left(k_{s s^{\prime}}\right) \text {. }
$$

Using again the linear independence in 9.6, we deduce that $\theta_{j, s^{\prime \prime}}$ annihilates $k_{s s^{\prime}}, l_{s s^{\prime}}, l_{s^{\prime} s}$. Using now (d), we see that $\theta_{j, s^{\prime \prime}}\left(m_{s s^{\prime}}\right)=0$. The lemma is proved.

10.12. Let $\mathscr{D}_{2}$ be the set of all derivations in $\mathscr{D}_{1}$ which map the subalgebra $A$ of $\tilde{A}$ to zero. Then $\mathscr{D}_{2}$ is a Lie subalgebra and an $A$-submodule of $\mathscr{D}_{1}$; moreover, the Lie algebra structure of $\mathscr{D}_{2}$ is compatible with the $A$-module structure so that $\mathscr{D}_{2}$ is an $A$-Lie algebra.

Lemma 10.13. Let $\xi=\sum_{j, s} a_{j, s} \theta_{j, s}$ be an element of $\mathscr{D}_{2}$ (here $a_{j, s} \in A$ ). For any $s \neq s^{\prime}$ in $S$, we have

$$
2 a_{-1, s} l_{s s^{\prime}}+2 a_{-1, s^{\prime}} l_{s^{\prime} s}+a_{0, s}+a_{0, s^{\prime}}=0 .
$$

Since $\xi$ annihilates any element of $A$, it annihilates in particular the elements $m_{s s^{\prime}}$ of $A$. We write the condition $m_{s s^{\prime}}^{-1} \xi\left(m_{s s^{\prime}}\right)=0$ explicitly, using the previous lemma; we find the desired identity.

10.14. We fix an element $s_{0} \in S$. Consider the isomorphism $H^{S} \cong H^{S}$ given by $\left(\gamma_{s}\right) \mapsto\left(\gamma_{s}^{\prime}\right)$ where $\gamma_{s_{0}}^{\prime}=\gamma_{s_{0}}$ and $\gamma_{s}^{\prime}=\gamma_{s_{0}}^{-1} \gamma_{s}$ for $s \neq s_{0}$.

Under this isomorphism, the vector field $\theta_{k, s_{0}}$ on the second copy of $H^{S}$ corresponds to a vector field $\sigma_{k}$ on the first copy of $H^{S}$.

It is clear that $\sigma_{k} \in \mathscr{D}_{2}$ and that

$$
\left[\sigma_{0}, \sigma_{-1}\right]=\sigma_{-1}, \quad\left[\sigma_{0}, \sigma_{1}\right]=-\sigma_{1}, \quad\left[\sigma_{1}, \sigma_{-1}\right]=2 \sigma_{0} .
$$

We have the following result. 
Lemma 10.15. The vector fields $\left\{\sigma_{k} \mid k \in\{1,0,-1\}\right\}$ form a (noncanonical) A-basis of the A-module $\mathscr{D}_{2}$.

The proof is immediate.

10.16. The vector field $\sigma_{k}$ can be written as an explicit linear combination of the vector fields $\theta_{k, s}$.

For this purpose, we write $k=c-d$ with $c, d \in[1,2]$. For any $a, b \in$ $[1,2]$ and any $s \in S$ we consider the following function $r_{a, b ; s} \in A$ :

$$
\left(\gamma_{t}\right) \mapsto(-1)^{a-c}\left(\gamma_{s_{0}}^{-1} \gamma_{s}\right)_{d b}\left(\gamma_{s}^{-1} \gamma_{s_{0}}\right)_{a c}
$$

where the subscripts $d b$ and $a c$ denote matrix coefficients; this is well defined.

We have

$$
\sigma_{k}=\sum_{a, b \in[1,2]} \sum_{s \in S} r_{a, b ; s} \theta_{a-b, s} .
$$

In particular, the right-hand side of (a) belongs to $\mathscr{D}_{2}$.

10.17. Assume, for example, that $S=\left\{s_{1}, s_{2}, s_{0}\right\}$; let $k=0$. Then, the righthand side of 10.16(a) evaluated at $\gamma_{s_{1}}=\left(\begin{array}{cc}0 & 1 \\ -1 & 1\end{array}\right), \gamma_{s_{2}}=\left(\begin{array}{cc}1 & -1 \\ 1 & 0\end{array}\right), \gamma_{s_{0}}=\left(\begin{array}{ll}1 & 0 \\ 0 & 1\end{array}\right)$ is

$$
\theta_{-1, s_{1}}-\theta_{0, s_{1}}+\theta_{1, s_{2}}-\theta_{0, s_{2}}+\theta_{0, s_{0}}
$$

10.18. On the other hand, if we assume that $S=\left\{s_{1}, s_{0}\right\}$ and $k=0$, then the right-hand side of 10.16(a) evaluated at $\gamma_{s_{1}}=\left(\begin{array}{cc}0 & -1 \\ 1 & 0\end{array}\right), \gamma_{s_{0}}=\left(\begin{array}{ll}1 & 0 \\ 0 & 1\end{array}\right)$ is

$$
-\theta_{0, s_{1}}+\theta_{0, s_{0}} \text {. }
$$

\section{SUgAWARA OPERATORS AND COINVARIANTS}

\subsection{We preserve the setup of 9.1, 9.2, 9.3.}

In addition, we assume that we are given a commutative $\mathbf{C}$-algebra $B$ and a homomorphism of $\mathbf{C}$-algebras $A \rightarrow B$.

We also assume that we are given a finite set $\&$ with a fixed element $t \in \boldsymbol{\$}$. Let $\mathbf{g}^{\prime}=\mathbf{g}^{\boldsymbol{*}}$, and let $l: \mathbf{g} \subset \mathbf{g}^{\prime}$ be the inclusion of the $t$-summand.

Moreover, we assume that for each $s \in S$ we are given a smooth $\widetilde{\mathbf{g}}_{B}^{\prime}$-module $\underline{V}_{s}$ with central charge $\kappa-h$.

The results in this section prepare the ground for the study of connections in $\S 12$. (For that case, we should take \& with one element.) There will be a second application of these results in $\S 17$; for that case we should take \& with two elements.

11.2. Let $Y=\bigotimes_{s \in S} \underline{V}_{s}$ (tensor product over $B$ ). Let $\mathscr{D}_{0}$ be as in 10.4, and let $\Lambda: \mathscr{D}_{0} \rightarrow \operatorname{End}_{B}(Y) \quad \partial \mapsto \Lambda_{\partial}$ be the unique $C$-linear map such that the following holds: for any $s \in S$ and any $k \in\{1,0,-1\}$, we have

$$
\Lambda_{\theta_{k, s}}=1 \otimes \cdots \otimes L_{k ; t} \otimes \cdots \otimes 1
$$


where $L_{k ; t}$ (on the $s$-position) represents the action of the Sugawara operator $L_{k}$ on $\underline{V}_{s}$, regarded as a $\widetilde{\mathbf{g}}_{B}$-module via the natural imbedding $\widetilde{\mathbf{g}}_{B} \subset \widetilde{\mathbf{g}}_{B}^{\prime}$ induced by $l: \mathbf{g} \rightarrow \mathbf{g}^{\prime}$ (see 1.15).

Using the commutation formulas 10.4 for $\theta_{k, s}$ and the commutation formulas 1.14(c) for the Sugawara operators, we see that

$\Lambda$ is a homomorphism of Lie algebras over $\mathbf{C}$.

Let $\mathscr{D}_{1}, \mathscr{D}_{2}$ be as in $10.5,10.12$.

Lemma 11.3. Let $\Lambda^{\prime}: \mathscr{D}_{1} \rightarrow \operatorname{End}_{B}(Y)$ be the unique A-linear map whose restriction to $\mathscr{D}_{0}$ is equal to $\Lambda$ (see 11.2); let $\Lambda^{\prime \prime}: \mathscr{D}_{2} \rightarrow \operatorname{End}_{B}(Y)$ be the restriction of $\Lambda^{\prime}$. Then $\Lambda^{\prime \prime}$ is a homomorphism of A-Lie algebras.

Let $\partial=\sum_{i} p_{i} \partial_{i}$ and $\partial^{\prime}=\sum_{j} p_{j}^{\prime} \partial_{j}^{\prime}$ be two elements of $\mathscr{D}_{2}$; here $p_{i}, p_{j}^{\prime} \in A$ and $\partial_{i}, \partial_{j}^{\prime} \in \mathscr{D}_{0}$. We have

$$
\left[\partial, \partial^{\prime}\right]=\sum_{i, j}\left(p_{i} \partial_{i}\left(p_{j}^{\prime}\right) \partial_{j}^{\prime}-p_{j}^{\prime} \partial_{j}^{\prime}\left(p_{i}\right) \partial_{i}+p_{i} p_{j}^{\prime}\left[\partial_{i}, \partial_{j}^{\prime}\right]\right) .
$$

Since $\partial, \partial^{\prime}$ are in $\mathscr{D}_{2}$, we have $\sum_{i} p_{i} \partial_{i}\left(p_{j}^{\prime}\right)=0, \quad \sum_{j} p_{j}^{\prime} \partial_{j}^{\prime}\left(p_{i}\right)=0$ and hence $\left[\partial, \partial^{\prime}\right]=\sum_{i, j} p_{i} p_{j}^{\prime}\left[\partial_{i}, \partial_{j}^{\prime}\right]$. Thus

$$
\left(\Lambda^{\prime \prime}\right)_{\left[\partial, \partial^{\prime}\right]}=\sum_{i, j} p_{i} p_{j}^{\prime} \Lambda_{\left[\partial_{i}, \partial_{j}^{\prime}\right]}=\sum_{i, j} p_{i} p_{j}^{\prime}\left[\Lambda_{\partial_{i}}, \Lambda_{\partial_{j}^{\prime}}\right]=\left[\left(\Lambda^{\prime \prime}\right)_{\partial},\left(\Lambda^{\prime \prime}\right)_{\partial^{\prime}}\right] .
$$

The lemma is proved.

11.4. Let $\Delta=A^{\prime} \otimes \mathbf{g}^{\prime}, \Delta_{B}=B \otimes_{A} \Delta$ be the Lie algebras defined in 9.9 (for $\mathbf{g}^{\prime}$ instead of $\mathbf{g}$ ). As in 9.9(b), we have a natural homomorphism of $B$-Lie algebras

$$
\Delta_{B} \rightarrow \widehat{\mathbf{g}}_{B}^{\prime S}
$$

11.5. We regard $Y$ as a $\widehat{\mathbf{g}}_{B}^{S}$-module as in 1.13 and, via $11.4(\mathrm{a})$, as a $\Delta_{B}$-module.

In the following lemma we assume given $s \in S, k \in\{1,0,-1\}, f \in A^{\prime}$, and $c \in \mathbf{g}^{\prime}$. We define $\widehat{c} \in \mathbf{g}^{\prime}$ to be the projection of $c$ onto the $t$-summand of $\mathbf{g}^{\prime}$, see 11.1. For any $\sigma \in S$, we write

$$
{ }^{\sigma} f=\sum_{n} p_{n, \sigma} \epsilon^{n}, \quad{ }^{\sigma}\left(\theta_{k, s} f\right)=\sum_{n} q_{n, \sigma} \epsilon^{n}
$$

where $p_{n, \sigma}, q_{n, \sigma} \in A$.

We define a $B$-linear map $\Theta_{k, s, \sigma}: Y \rightarrow Y$ by

$$
\Theta_{k, s, \sigma}=1 \otimes 1 \otimes \cdots \otimes\left(\sum_{n} \theta_{k, s}\left(p_{n, \sigma}\right) \epsilon^{n} \hat{c}\right) \otimes \cdots \otimes 1 \otimes 1
$$

(the element $\left(\sum_{n} \theta_{k, s}\left(p_{n, \sigma}\right) \epsilon^{n} \widehat{c}\right)$ acts on the $\sigma$-factor). 
Lemma 11.6. For any $y \in Y$, we have

$$
\Lambda_{\theta_{k, s}}((f c) y)+\sum_{\sigma \in S} \boldsymbol{\Theta}_{k, s, \sigma} y=(f c)\left(\Lambda_{\theta_{k, s}} y\right)+\left(\left(\theta_{k, s} f\right) \widehat{c}\right) y
$$

We may assume that $y=\otimes_{s^{\prime}} y_{s^{\prime}}$ with $y_{s^{\prime}} \in \underline{V}_{s^{\prime}}$. Using the definitions we see that the left-hand side of (a) is a sum of terms, one for each $\sigma \in S$; the term corresponding to $\sigma=s$ is

$$
\left(L_{k ; t}\left(\sum_{n} p_{n, s} \epsilon^{n} c y_{s}\right)\right) \otimes\left(\bigotimes_{s^{\prime} \neq s} y_{s^{\prime}}\right)+\left(\sum_{n} \theta_{k, s}\left(p_{n, s}\right) \epsilon^{n} \widehat{c} y_{s}\right) \otimes\left(\bigotimes_{s^{\prime} \neq s} y_{s^{\prime}}\right)
$$

the term corresponding to $\sigma \neq s$ is

$$
\left(\sum_{n} p_{n, \sigma} \epsilon^{n} c y_{\sigma}\right) \otimes L_{k ; t} y_{s} \otimes\left(\bigotimes_{s^{\prime} \neq s, \sigma} y_{s^{\prime}}\right)+\left(\sum_{n} \theta_{k, s}\left(p_{n, \sigma}\right) \epsilon^{n} \widehat{c} y_{\sigma}\right) \otimes\left(\bigotimes_{s^{\prime} \neq \sigma} y_{s^{\prime}}\right) \text {. }
$$

The right-hand side of (a) is a sum of terms, one for each $\sigma \in S$; the term corresponding to $\sigma=s$ is

$$
\left(\sum_{n} p_{n, s} \epsilon^{n} c\left(L_{k ; t} y_{s}\right)+\sum_{n} q_{n, s} \epsilon^{n} \widehat{c} y_{s}\right) \otimes\left(\bigotimes_{s^{\prime} \neq s} y_{s^{\prime}}\right)
$$

the term corresponding to $\sigma \neq s$ is

$$
\left(\sum_{n} p_{n, \sigma}\left(\epsilon^{n} c\right) y_{\sigma}\right) \otimes L_{k} y_{s} \otimes\left(\bigotimes_{s^{\prime} \neq s, \sigma} y_{s^{\prime}}\right)+\left(\sum_{n} q_{n, \sigma} \epsilon^{n} \widehat{c} y_{\sigma}\right) \otimes\left(\bigotimes_{s^{\prime} \neq \sigma} y_{s^{\prime}}\right)
$$

Using the identity $q_{n, \sigma}+\delta_{s \sigma}(n-k) p_{n-k, \sigma}=\theta_{k, s}\left(p_{n, \sigma}\right)$ (see 10.8) we can cancel several of the terms above; it remains to verify the following identity:

$$
L_{k ; t}\left(\sum_{n} p_{n, s} \epsilon^{n} c y_{s}\right)+\sum_{n}(n-k) p_{n-k, s} \epsilon^{n} \widehat{c} y_{s}=\sum_{n} p_{n, s} \epsilon^{n} c\left(L_{k ; t} y_{s}\right)
$$

for all $y_{s} \in \underline{V}_{s}$. This follows from $\left(\epsilon^{n} c\right)\left(L_{k ; t} y_{s}\right)-L_{k ; t}\left(\epsilon^{n} c y_{s}\right)=n \epsilon^{n+k} \widehat{c} y_{s}$. (In the right-hand side we have $\widehat{c}$ and not $c$, as in $1.14(\mathrm{~b})$, since $L_{k ; t}$ acts only through the $t$-component of $\widetilde{\mathbf{g}}_{B}^{\prime}$.)

Lemma 11.7. For any $y \in Y$ and any $\partial \in \mathscr{D}_{2}$, we have

$$
\Lambda_{\partial}^{\prime \prime}((f c) y)=(f c)\left(\Lambda_{\partial}^{\prime \prime} y\right)+(\partial(f) \widehat{c})(y) .
$$

We can write $\partial=\sum_{k, s} a_{k, s} \theta_{k, s}$ with $a_{k, s} \in A$. Using the previous lemma we see that it is enough to show that for any $\boldsymbol{\sigma}$ we have $\sum_{k, s} a_{k, s} \boldsymbol{\Theta}_{k, s, \sigma}(y)=0$.

This follows from the definition of $\Theta_{k, s}$, since $\sum_{k, s} a_{k, s} \theta_{k, s}\left(p_{n, \sigma}\right)=$ $\partial\left(p_{n, \sigma}\right)=0$, by the definition of $\mathscr{D}_{2}$.

Lemma 11.8. For any $\partial \in \mathscr{D}_{2}$, the endomorphism $\Lambda_{\partial}^{\prime \prime}$ of $Y$ maps the $B$ submodule $\Delta_{B} Y$ into itself. Hence it induces an endomorphism (denoted again $\left.\Lambda_{\partial}^{\prime \prime}\right)$ of the B-module of coinvariants $Y / \Delta Y$.

This follows immediately from the previous lemma. 
Lemma 11.9. Let $\xi \in \mathscr{D}_{2}$, and let $y \in Y$ be of the form $y=\bigotimes_{s} y_{s}$ where $y_{s} \in \underline{V}_{s}(1)$ for all $s$. We have $\Lambda_{\xi}^{\prime \prime}(y) \in \Delta_{B} Y$.

Since $y_{s} \in \underline{V}_{s}(1)$, we see from the definition of the Sugawara operators that $L_{1 ; t}\left(y_{s}\right)=0, \quad L_{0 ; t}\left(y_{s}\right)=\frac{1}{2 \kappa} \sum_{p} c_{p} c_{p} y_{s}, \quad L_{-1 ; t}\left(y_{s}\right)=\frac{1}{\kappa} \sum_{p}\left(\epsilon^{-1} c_{p}\right)\left(c_{p} y_{s}\right)$ where $\left(c_{p}\right)$ is a basis of $\mathbf{g}$ such that $\left(c_{p}, c_{p^{\prime}}\right)=\delta_{p p^{\prime}}$. (We identify $\mathbf{g}$ with a Lie subalgebra of $\mathbf{g}^{\prime}$ via $l$, see 11.1.) Hence we have $\Lambda_{\theta_{1, s}}(y)=0$.

Moreover, $\Lambda_{\theta_{0, s}}(y)=\frac{1}{2 \kappa} \sum_{p}\left(c_{p} c_{p} y_{s}\right) \otimes\left(\bigotimes_{\sigma \neq s} y_{\sigma}\right)$.

Applying the element $c_{p} \in \mathbf{g}$ (regarded as element of $\Delta$ ) to the vector $\left(c_{p} y_{s}\right) \otimes$ $\left(\otimes_{\sigma \neq s} y_{\sigma}\right)$ we obtain

$$
\left(c_{p} c_{p} y_{s}\right) \otimes\left(\bigotimes_{\sigma \neq s} y_{\sigma}\right)+\sum_{s^{\prime} \neq s}\left(c_{p} y_{s}\right) \otimes\left(c_{p} y_{s^{\prime}}\right) \otimes\left(\bigotimes_{\sigma \neq s, s^{\prime}} y_{\sigma}\right) .
$$

Hence $\Lambda_{\theta_{0, s}}(y)$ is equal to $-\frac{1}{2 \kappa} \sum_{p} \sum_{s \neq s^{\prime}}\left(c_{p} y_{s}\right) \otimes\left(c_{p} y_{s^{\prime}}\right) \otimes\left(\otimes_{\sigma \neq s, s^{\prime}} y_{\sigma}\right)$ plus an element of $\Delta_{B} Y$.

Next, we have $\Lambda_{\theta_{-1, s}}(y)=\frac{1}{\kappa} \sum_{p}\left(\epsilon^{-1} c_{p}\right)\left(c_{p} y_{s}\right) \otimes\left(\bigotimes_{\sigma \neq s} y_{\sigma}\right)$.

Applying the element $f_{s} c_{p} \in \Delta$ to the vector $\left(c_{p} y_{s}\right) \otimes\left(\bigotimes_{\sigma \neq s} y_{\sigma}\right)$, we obtain

$$
\left(\left(\epsilon^{-1} c_{p}\right) c_{p} y_{s}\right) \otimes\left(\bigotimes_{\sigma \neq s} y_{\sigma}\right)+\sum_{s^{\prime} \neq s}\left(c_{p} y_{s}\right) \otimes\left(l_{s s^{\prime}} c_{p} y_{s^{\prime}}\right) \otimes\left(\bigotimes_{\sigma \neq s, s^{\prime}} y_{\sigma}\right) .
$$

(Here we use that, for $s^{\prime} \neq s$, we have ${ }^{s^{\prime}} f_{s}-l_{s s^{\prime}} \in \epsilon A[[\epsilon]]$ so that $\left(^{s^{\prime}} f_{s}-l_{s s^{\prime}}\right) c_{p} y_{s}=0$ for $y_{s} \in \underline{V}_{s}(1)$.) Hence $\Lambda_{\theta_{-1, s}}(y)$ is equal to

$$
-\frac{1}{\kappa} \sum_{p} \sum_{s \neq s^{\prime}} l_{s s^{\prime}}\left(c_{p} y_{s}\right) \otimes\left(c_{p} y_{s^{\prime}}\right) \otimes\left(\bigotimes_{\sigma \neq s, s^{\prime}} y_{\sigma}\right)
$$

plus an element of $\Delta_{B} Y$.

We now write $\xi=\sum_{k, s} a_{k, s} \theta_{k, s}$ with $a_{k, s} \in A$. From the previous formulas we see that $\Lambda_{\xi}^{\prime \prime}(y)$ is equal to

$$
-\frac{1}{2 \kappa} \sum_{p} \sum_{s \neq s^{\prime}} r_{s, s^{\prime}}\left(c_{p} y_{s}\right) \otimes\left(c_{p} y_{s^{\prime}}\right) \otimes\left(\bigotimes_{\sigma \neq s, s^{\prime}} y_{\sigma}\right)
$$

plus an element of $\Delta_{B} Y$; here, the second sum is taken over unordered pairs $s \neq s^{\prime}$ (unlike in the earlier sums) and $r_{s, s^{\prime}}=2 a_{-1, s} l_{s s^{\prime}}+2 a_{-1, s^{\prime}} l_{s^{\prime} s}+a_{0, s}+a_{0, s^{\prime}}$. The last expression is zero, by 10.13 . The lemma is proved.

Lemma 11.10. Assume that for each $s \in S$ there exists $N_{s} \geq 1$ such that $\underline{V}_{s}\left(N_{s}\right)$ generates $\underline{V}_{S}$ as a $\widetilde{\mathbf{g}}_{B}^{\prime}$-module. Then for any $\xi \in \mathscr{D}_{2}$ we have $\Lambda_{\xi}^{\prime \prime N}(Y) \subset \Delta_{B} Y$, where $N=\prod_{s} N_{s}$. 
Assume that $N \geq 2$ and that the lemma is already known for $N$ replaced by $N^{\prime}$ with $1 \leq N^{\prime}<N$. Then for some $s=s_{0}$ we have $N_{s_{0}} \geq 2$. Let $\underline{V}_{s_{0}}^{\prime}$ be the $\widetilde{\mathbf{g}}_{B}^{\prime}$-submodule of $\underline{V}_{s_{0}}$ generated by $\underline{V}_{s_{0}}\left(N_{s_{0}}-1\right)$, and let $\underline{V}_{s_{0}}^{\prime \prime}=\underline{V}_{s_{0}} / \underline{V}_{s_{0}}^{\prime}$.

The induction hypothesis is applicable when $V_{s_{0}}$ is replaced by $V_{s_{0}}^{\prime}$ (resp. by $\left.V_{s_{0}}^{\prime \prime}\right)$ and the other $V_{s}$ are left unchanged; indeed, $\underline{V}_{s_{0}}^{\prime}$ is generated by $\underline{V}_{s_{0}}^{\prime}\left(N_{s_{0}}-1\right)$ and $\underline{V}_{s_{0}}^{\prime \prime}$ is generated by $\underline{V}_{s_{0}}^{\prime \prime}(1)$ (as $\widetilde{\mathbf{g}}_{B}^{\prime}$-modules).

Let $Y_{0}=\otimes_{s: s \neq s_{0}} \underline{V}_{s}$ (tensor products over $B$ ). Then $Y=\underline{V}_{s_{0}} \otimes_{B} \underline{Y}_{0}$. We set $Y^{\prime}=V_{s_{0}}^{\prime} \otimes_{B} Y_{0}, Y^{\prime \prime}=\underline{V}_{s_{0}}^{\prime \prime} \otimes_{B} Y_{0}$.

We have an exact sequence $0 \rightarrow \underline{V}_{s_{0}}^{\prime} \rightarrow \underline{V}_{s_{0}} \rightarrow \underline{V}_{s_{0}}^{\prime \prime} \rightarrow 0$. This induces an exact sequence

$$
Y^{\prime} \stackrel{j}{\longrightarrow} Y \stackrel{\rho}{\longrightarrow} Y^{\prime \prime} \longrightarrow 0
$$

Let $N^{\prime \prime}=N / N_{s_{0}}, N^{\prime}=\left(N_{s_{0}}-1\right) N^{\prime \prime}$. Let $y \in Y$. By the induction hypothesis, $\rho\left(\Lambda_{\xi}^{\prime \prime N^{\prime \prime}}(y)\right)=\Lambda_{\xi}^{\prime \prime} N^{\prime \prime} \rho(y)$ is contained in $\Delta_{B} Y^{\prime \prime}$; hence, by the surjectivity of $\rho$, it is contained in $\rho\left(\Delta_{B} Y\right)$. From the exactness of (c) it follows that $\Lambda_{\xi}^{\prime \prime N^{\prime \prime}}(y)$ is the sum of an element of $\Delta_{B} Y$ and an element in the image of $Y^{\prime}$. This last element is annihilated by $\Lambda_{\xi}^{\prime \prime N^{\prime}}$, by the induction hypothesis. Hence $\Lambda_{\xi}^{\prime \prime N^{\prime}+N^{\prime \prime}}(y)$ is contained in $\Lambda_{\xi}^{\prime \prime N^{\prime}}\left(\Delta_{B} Y\right)$ and this last set is contained in $\Delta_{B} Y$, by 11.8. Thus, $\Lambda_{\xi}^{\prime \prime N}(y)$ is contained in $\Delta_{B} Y$. (Note that $N=N^{\prime}+N^{\prime \prime}$.) Thus the lemma holds, under our inductive assumption.

We are therefore reduced to verifying the lemma in the special case where $N=1$. In this case, we have that $Y$ is the sum of $\Delta_{B} Y$ and of the image of $\otimes_{s} \underline{V}_{s}(1)$ (see 9.12) and $\Lambda_{\xi}^{\prime \prime}$ is zero on the last submodule (see 11.9). It follows that $\Lambda_{\xi}^{\prime \prime}(Y)=\Lambda_{\xi}^{\prime \prime}\left(\Delta_{B} Y\right)$ and this set is contained in $\Delta_{B} Y$, by 11.8. The lemma is proved.

Proposition 11.11. Assume that for each $s \in S$ there exists $N_{s} \geq 1$ such that $\underline{V}_{s}\left(N_{s}\right)$ generates $\underline{V}_{s}$ as a $\widetilde{\mathbf{g}}_{B}^{\prime}$-module. Then $\Lambda_{\xi}^{\prime \prime}(Y) \subset \Delta_{B} Y$ for any $\xi \in \mathscr{D}_{2}$.

Since $\mathscr{D}_{2}$ is generated as an $A$-module by $\sigma_{k}(k=1,0,-1)$ (see 10.15), it is enough to prove the lemma in the case where $\xi=\sigma_{k}$. We may regard $\Lambda_{\sigma_{k}}^{\prime \prime}$ as $B$-linear maps $Y / \Delta_{B} Y \rightarrow Y / \Delta_{B} Y$ (see 11.8). These maps satisfy the commutation relations 10.14 between the $\sigma_{k}$ (see 11.3). Thus they define a complex representation of the Lie algebra $\operatorname{sl}_{2}(\mathbf{C})$. In particular, for any vector $\bar{y} \in Y / \Delta_{B} Y$, the $\mathrm{C}$-vector subspace $P$ of $Y / \Delta_{B} Y$ spanned by the elements

$$
\left(\Lambda_{\sigma_{1}}^{\prime \prime}\right)^{a}\left(\Lambda_{\sigma_{0}}^{\prime \prime}\right)^{b}\left(\Lambda_{\sigma_{-1}}^{\prime \prime}\right)^{c} \bar{y}
$$

(for various $a, b, c \in \mathbf{N}$ ) is stable under this action of $\mathbf{s l}_{2}(\mathbf{C})$. The endomorphisms $\Lambda_{\sigma_{k}}^{\prime \prime}$ of $Y / \Delta_{B} Y$ are nilpotent (11.10) and hence only finitely many of the elements (a) are nonzero. Thus, $P$ is a finite-dimensional complex representation of $\mathbf{s l}_{2}(\mathbf{C})$ in which the three generators $\sigma_{k}$ act nilpotently. As it is 
well known, this implies that the $\mathbf{s l}_{2}(\mathbf{C})$-action is zero on $P$. In particular, we have $\Lambda_{\sigma_{k}}^{\prime \prime}(\bar{y})=0$. Since $\bar{y}$ was arbitrary, we have $\Lambda_{\sigma_{k}}^{\prime \prime}=0$ on $Y / \Delta_{B} Y$. The proposition is proved.

Corollary 11.12. In the setup of 10.16 and with the assumption of the proposition, we have

$$
\sum_{a, b \in[1,2]} \sum_{s \in S} r_{a, b ; s} \Lambda_{\theta_{a-b, s}}(y) \subset \Delta_{B} Y
$$

for all $y \in Y$.

\section{ConNections}

In this section we define an integrable connection on a space of coinvariants in a tensor product, using Sugawara operators.

12.1. We preserve the setup of 11.1 and reinforce the assumptions there by the following ones:

$$
B=A, \mathbf{g}^{\prime}=\mathbf{g} \text {, and } \underline{V}_{s}=A \otimes V_{s} \text { where } V_{s} \text { are given objects of } \mathscr{O}_{\kappa} .
$$

We may identify $V_{s}$ with a subspace of $\underline{V}_{s}$ via $y \rightarrow 1 \otimes y$.

12.2. We begin with some generalities on connections.

Let $\mathscr{A}$ be a commutative $\mathbf{C}$-algebra with 1 . Following Grothendieck [G, $\S 20.5]$, we define $\Omega_{\mathscr{A}}^{1}=\mathscr{I} / \mathscr{I}^{2}$ where $\mathscr{I}$ is the kernel of the natural algebra homomorphism $\mathscr{A} \otimes_{\mathbf{C}} \mathscr{A} \rightarrow A \quad\left(a \otimes a^{\prime} \mapsto a a^{\prime}\right)$; thus $\mathscr{I}$ is an ideal of $\mathscr{A} \otimes_{\mathbf{C}} \mathscr{A}$. Then $\Omega_{\mathscr{A}}^{1}$ is naturally an $A$-module: for $a \in A$ and $\sum_{i} a_{i}^{\prime} \otimes a_{i}^{\prime \prime} \in \mathscr{I}$ we set $a\left(\sum_{i} a_{i}^{\prime} \otimes a_{i}^{\prime \prime}\right)=\sum_{i} a a_{i}^{\prime} \otimes a_{i}^{\prime \prime}=\sum_{i} a_{i}^{\prime} \otimes a a_{i}^{\prime \prime}$ (the second equality holds modulo $\mathscr{I}^{2}$ ). Let $d: \mathscr{A} \rightarrow \Omega_{\mathscr{A}}^{1}$ be the $\mathscr{A}$-module homomorphism given by $d(a)=a \otimes 1-1 \otimes a$.

Let $M$ be an $\mathscr{A}$-module. A connection on $M$ is, by definition, a C-linear map $\nabla: M \rightarrow \Omega_{\mathscr{A}}^{1} \otimes_{\mathscr{A}} M$ such that $\nabla(a m)=d(a) \otimes m+a \nabla(m)$ for all $a \in \mathscr{A}$ and $m \in M$.

Let Der $\mathscr{A}$ be the set of algebra derivation of $\mathscr{A}$. It is naturally a (left) $\mathscr{A}$ module and a C-Lie algebra, with bracket given by the commutator of operators.

From the definition, it follows that $f \mapsto f d$ defines an isomorphism of $\mathscr{A}$-modules $\operatorname{Hom}_{\mathscr{A}}\left(\Omega_{\mathscr{A}}^{1}, \mathscr{A}\right) \cong \operatorname{Der} \mathscr{A}$.

12.3. To a connection $\nabla$ on an $\mathscr{A}$-module $M$, we can associate a map

$$
\operatorname{Der} \mathscr{A} \rightarrow \operatorname{End}_{\mathbf{C}} M \quad\left(\partial \mapsto \nabla_{\partial}\right)
$$

as follows: for any $\partial \in \operatorname{Der} \mathscr{A}, \nabla_{\partial}: M \rightarrow M$ is defined as the composition of $\nabla: M \rightarrow \Omega_{\mathscr{A}}^{1} \otimes_{\mathscr{A}} M$ with $\partial \otimes 1_{M}: \Omega_{\mathscr{A}}^{1} \otimes_{\mathscr{A}} M \rightarrow \mathscr{A} \otimes_{\mathscr{A}} M=M$ (we regard $\partial$ as an element of $\operatorname{Hom}_{\mathscr{A}}\left(\Omega_{\mathscr{A}}^{1}, \mathscr{A}\right)$, as above).

We have the following identities:

$$
\begin{gathered}
\nabla_{\partial}(a m)=\partial(a) m+a \nabla_{\partial}(m), \\
\nabla_{a \partial+a^{\prime} \partial^{\prime}}(m)=a \nabla_{\partial}(m)+a^{\prime} \nabla_{\partial^{\prime}}(m)
\end{gathered}
$$

for all $a, a^{\prime} \in \mathscr{A}, \partial, \partial^{\prime} \in \operatorname{Der} \mathscr{A}$, and $m \in M$. 
12.4. From now on we will restrict ourselves to the case where $\mathscr{A}$ has the following property:

$$
\Omega_{\mathscr{A}}^{1} \text { is a reflexive } \mathscr{A} \text {-module }
$$

This property is satisfied if, for example, $\mathscr{A}$ is the coordinate ring of a smooth affine variety over $\mathbf{C}$ or a regular finitely generated local $\mathbf{C}$-algebra. Another example is the Artin algebra $\mathscr{A}=\mathbf{C}[\epsilon] /\left(\epsilon^{n}\right)$ for $n \geq 1$. In this case, any finitely generated $A$-module is reflexive and, in particular, (a) holds (although $\Omega_{\mathscr{A}}^{1}$ is not free over $\mathscr{A}$.)

12.5. Under the assumption $12.4(\mathrm{a}), \Omega_{\mathscr{A}}^{1}$ can be reconstructed from Der $\mathscr{A}$ as $\Omega_{\mathscr{A}}^{1}=\operatorname{Hom}_{\mathscr{A}}(\operatorname{Der} \mathscr{A}, \mathscr{A})$, and we see that giving a connection $\nabla$ on an $\mathscr{A}$-module $M$ is the same as giving a $\mathrm{C}$-linear map

$$
\operatorname{Der} \mathscr{A} \rightarrow \operatorname{End}_{\mathrm{C}} M \quad\left(\partial \mapsto \nabla_{\partial}\right)
$$

satisfying $12.3(\mathrm{~b}),(\mathrm{c})$; this last map will be also referred to as a connection.

We say that this connection is integrable if (a) is a Lie algebra homomorphism (where End $_{\mathbf{C}} M$ is a Lie algebra with bracket given as commutator of operators).

\section{Lemma 12.6. Assume that either}

(a) $\mathscr{A}$ is the coordinate ring of a smooth affine variety over $\mathbf{C}$, or

(b) $\mathscr{A}$ is a regular finitely generated local C-algebra.

Let $M$ be a finitely generated $\mathscr{A}$-module which admits a connection $\nabla$. Then $M$ is a projective $\mathscr{A}$-module.

The proof will be given in 12.13 .

12.7. We return to the setup in 12.1. The $A$-module $Y=\bigotimes_{s \in S} \underline{V}_{s}$ (tensor product over $A$ ) is the same as $A \otimes\left(\otimes_{s} V_{s}\right)$ (tensor products over $\mathbf{C}$ ); it has an obvious connection $\nabla^{0}$, given by

$$
\nabla_{\partial}^{0}(f y)=\partial(f) y
$$

for all $f \in A$ and $y \in \bigotimes_{s} V_{s}$ (tensor product over $\mathbf{C}$ ). This connection is integrable.

Let $\nabla^{1}: \mathscr{D}_{1} \rightarrow \operatorname{End}_{\mathbf{C}}(Y)$ be the unique $A$-linear map such that

$$
\nabla_{\partial}^{1}=\nabla^{0} \pi(\partial)+\Lambda_{\partial}^{\prime}: Y \rightarrow Y
$$

for any $\partial \in \mathscr{D}_{1}$; here, $\pi$ denotes the canonical map $\mathscr{D}_{1} \rightarrow \operatorname{Der} A$ (see 10.5) and $\Lambda^{\prime}$ is as in 11.3.

Lemma 12.8. (a) For any $y \in Y$, any $f \in A$, and any $\partial \in \mathscr{D}_{1}$, we have $\nabla_{\partial}^{1}(f y)=f \nabla_{\partial}^{1}(y)+((\pi \partial) f) y$.

(b) $\nabla^{1}$ is a homomorphism of $\mathbf{C}$-Lie algebras.

(a) follows from the definitions. Using (a) we see that to prove (b) it is enough to show that the restriction of $\nabla^{1}$ to $\mathscr{D}_{0}$ is a Lie algebra homomorphism. 
Using $11.2(\mathrm{a})$ and the integrability of the connection $\nabla^{0}$, we see that it is enough to verify that for any $\partial, \partial^{\prime} \in \mathscr{D}_{0}$ we have $\nabla_{\partial}^{0} \Lambda_{\partial^{\prime}}=\Lambda_{\partial^{\prime}} \nabla_{\partial}^{0}$ as endomorphisms of $Y$.

This follows from the definitions, since the Sugawara operators have "constant" coefficients and such coefficients are annihilated by all derivations of $A$.

The following result describes the commutator of this endomorphism $f c$ of $Y \quad\left(f c \in \Delta, f \in A^{\prime}, c \in \mathbf{g}\right)$ given by the $\Delta$-module structure, with the endomorphism $\nabla_{\theta_{k, s}}^{1}$.

Lemma 12.9. For any $y \in Y$, we have

$$
\nabla_{\theta_{k, s}}^{1}((f c) y)=(f c)\left(\nabla_{\theta_{k, s}}^{1} y\right)+\left(\left(\theta_{k, s} f\right) c\right) y
$$

We have, using the definitions,

$$
\nabla_{\theta_{k, s}}^{0}((f c) y)=(f c)\left(\nabla_{\theta_{k, s}}^{0} y\right)+\sum_{\sigma \in S} \boldsymbol{\Theta}_{k, s, \sigma} y
$$

where $\Theta_{k, s, \sigma} y$ is as in 11.5. We add this term-by-term with the identity

$$
\Lambda_{\theta_{k, s}}((f c) y)+\sum_{\sigma \in S} \boldsymbol{\Theta}_{k, s, \sigma} y=(f c)\left(\Lambda_{\theta_{k, s}} y\right)+\left(\left(\theta_{k, s} f\right) c\right) y
$$

(see 11.6; the element $\widehat{c}$ of 11.6 is now $c$ ). The lemma follows.

Lemma 12.10. (a) For any $\partial \in \mathscr{D}_{1}$, the endomorphism $\nabla_{\partial}^{1}$ of $Y$ maps the subspace $\Delta Y$ into itself. Hence it induces a C-linear endomorphism (denoted again $\nabla_{\partial}^{1}$ ) of $Y / \Delta Y$.

(b) If $\partial \in \mathscr{D}_{2}$, then the endomorphism $\nabla_{\partial}^{1}$ of $Y / \Delta Y$ is zero.

The subspace $\Delta Y$ is an $A$-submodule of $Y$; hence, it is enough to prove (a) in the case where $\partial$ is of the form $\theta_{k, s}$. We apply 12.9 (a) with $y \in Y, c \in \mathbf{g}$, and $f \in A^{\prime}$. The right-hand side of that equality is in $\Delta Y$ since $\theta_{k, s} f \in A^{\prime}$. Hence so is the left-hand side; (a) follows.

We prove (b). Let $\partial \in \mathscr{D}_{2}$. We have $\nabla_{\pi(\partial)}^{0}(Y)=0$ (since $\pi(\partial)=0$ ) and $\Lambda_{\partial}^{\prime}(Y) \subset \Delta Y$ (by 11.11). This proves (b).

12.11. The Lie algebra homomorphism $\mathscr{D}_{1} \rightarrow \operatorname{End}_{\mathbf{C}}(Y / \Delta Y)$ given by $\partial \mapsto \nabla_{\partial}^{1}$ (see $12.10(\mathrm{a})$ ) is zero on the ideal $\mathscr{D}_{2}($ see $12.10(\mathrm{~b}))$ and hence it factors through a Lie algebra homomorphism

$$
\operatorname{Der}(A)=\mathscr{D}_{1} / \mathscr{D}_{2} \rightarrow \operatorname{End}_{\mathbf{C}}(Y / \Delta Y)
$$

This is an integrable connection on the $A$-module $Y / \Delta Y$.

Proposition 12.12. The A-module $Y / \Delta Y$ is projective, of finite rank.

The fact that it is a finitely generated $A$-module follows from 9.16; the fact that it is projective follows from 12.6.

12.13. Proof of Lemma 12.6. If 12.6 (b) holds and $\mathscr{A}$ is as in $12.6(\mathrm{a})$, then the localization of $M$ at any maximal ideal of $\mathscr{A}$ admits an induced connection 
and hence is projective; it follows that $M$ itself is projective. It remains to prove the lemma under the assumption of $12.6(\mathrm{~b})$.

We denote by $J$ the unique maximal ideal of $\mathscr{A}$. We can find generators $t_{1}, \ldots, t_{d}$ of the ideal $J$ such that their images in $J / J^{2}$ form a basis of $J / J^{2}$ as a $\mathscr{A} / J=\mathrm{C}$-vector space. We can find elements $m_{1}, \ldots, m_{p}$ in $M$ such that their images in $M / J M$ form a basis of $M / J M$ as a $\mathrm{C}$-vector space. Now the elements $d\left(t_{1}\right), \ldots, d\left(t_{d}\right)$ form a basis of $\Omega_{\mathscr{A}}^{1}$ as an $\mathscr{A}$-module; and hence there are well-defined C-linear maps $\nabla_{k}: M \rightarrow M(k=1, \ldots, d)$ such that $\nabla(m)=\sum_{k} d\left(t_{k}\right) \otimes \nabla_{k}(m)$ for all $m \in M$; we have $\nabla_{k}(a m)=\partial_{k} a m+a \nabla_{k}(m)$ for all $a \in \mathscr{A}, m \in M$ where $\partial_{k}: \mathscr{A} \rightarrow \mathscr{A}$ is the derivation defined by $d(a)=\sum_{k} \partial_{k}(a) d\left(t_{k}\right)$.

Note that $\partial_{k}\left(t_{k^{\prime}}\right)=\delta_{k k^{\prime}}$. It follows easily that:

If $n \geq 1$ and $a \in J^{n}$ are such that $\partial_{k}(a) \in J^{n}$ for all $k$, then $a \in J^{n+1}$

We will show by induction on $n \geq 1$ that the following property holds: If $a_{1}, \ldots a_{p} \in \mathscr{A}$ satisfy $a_{1} m_{1}+\cdots+a_{p} m_{p} \in J^{n} M$, then $a_{k} \in J^{n}$ for all $k$.

Let $\bar{m}, \bar{a}$ be the image of $m \in M, a \in \mathscr{A}$ in $M / J M, \mathscr{A} / J \mathscr{A}$ respectively. We first verify $\left(\mathbf{P}_{1}\right)$. Let $a_{1}, \ldots, a_{p} \in \mathscr{A}$ be such that $a_{1} m_{1}+\cdots+a_{p} m_{p} \in$ $J M$. Then $\bar{a}_{1} \bar{m}_{1}+\cdots+\bar{a}_{p} \bar{m}_{p}=0$, and hence by the linear independence of $\bar{m}_{1}, \ldots, \bar{m}_{p}$ we have $\bar{a}_{1}=\cdots=\bar{a}_{p}=0$ so that $a_{1}, \ldots, a_{p} \in J$ and $\left(\mathrm{P}_{1}\right)$ is verified.

Assume now that $\left(\mathrm{P}_{n}\right)$ holds for some $n \geq 1$; we show that $\left(\mathrm{P}_{n+1}\right)$ must also hold. Let $a_{1}, \ldots a_{p} \in \mathscr{A}$ be such that $a_{1} m_{1}+\cdots+a_{p} m_{p} \in J^{n+1} M$. Applying $\nabla_{j}$ and using $\nabla_{j}\left(J^{n+1} M\right) \subset J^{n} M$, we see that $\sum_{k} \partial_{j}\left(a_{k}\right) m_{k}+\sum_{k} a_{k} \nabla_{j}\left(m_{k}\right) \in$ $J^{n} M$.

From $\left(\mathbf{P}_{n}\right)$ we see that $a_{k} \in J^{n}$ for all $k$; hence, $\sum_{k} \partial_{j}\left(a_{k}\right) m_{k} \in J^{n} M$. Using again $\left(\mathbf{P}_{n}\right)$ we deduce that $\partial_{j}\left(a_{k}\right) \in J^{n}$ for all $j, k$. Using now (c) we see that $a_{k} \in J^{n+1}$ for all $k$; thus, $\left(\mathrm{P}_{n+1}\right)$ holds. The property $\left(\mathrm{P}_{n}\right)$ is proved by induction.

We will show that the elements $m_{1}, \ldots, m_{p}$ form a basis of $M$ as an $\mathscr{A}$ module. They certainly generate $M$ as an $\mathscr{A}$-module, by Nakayama. It remains to show that given a relation $a_{1} m_{1}+\cdots+a_{p} m_{p}=0$ with $a_{1}, \ldots, a_{p} \in \mathscr{A}$ we must have $a_{1}=\cdots=a_{p}=0$. Our relation implies, by $\left(\mathrm{P}_{n}\right)$, that $a_{k} \in J^{n}$ for all $n \geq 1$. Since $\bigcap_{n} J^{n}=0$, it follows that $a_{k}=0$ for all $k$. The lemma is proved.

\section{TENSOR PRODUCT AND COINVARIANTS}

In this section we will define something like a base point for the space $\mathscr{V}$ of 9.2 in the case where $S$ has a given cyclic order. More precisely, we will define a contractible real submanifold of $\underline{\mathscr{V}}$. For our purposes, this is as good as a base point. It will allow us to extend the definition of $\left\langle V, V^{\prime}\right\rangle$ (see 2.32) to more than two objects of $\mathscr{O}_{\kappa}$. 
13.1. Let $S$ be a finite set such that $|S|=r \geq 2$, with a given cyclic order. For convenience, we write its elements as $1,2, \ldots, r$ where $1<2<\cdots<r<1$ is the cyclic order.

Let $P_{\mathbf{R}}^{1}=\mathbf{R} \cup \infty$ be the real projective line (a real analytic submanifold of $P^{1}$ isomorphic to a circle). We shall regard the interval $(0, \delta)$ in $\mathbf{R}$ (where $\left.\delta \in \mathbf{R}_{>0}\right)$ as a subset of $P_{\mathbf{R}}^{1}$.

We define a subset $\mathscr{V}_{0}$ of $P G L_{2}(\mathbf{R})^{S}$ as follows.

If $r=2, \mathscr{V}_{0}$ consists of all $\left(\gamma_{i}\right)_{i \in S} \in P G L_{2}(\mathbf{R})^{S}$ such that $\gamma_{1}(0), \gamma_{2}(0)$ are distinct points of $P_{\mathbf{R}}^{1}$ and $\gamma_{1}^{-1} \gamma_{2}$ is in the image of $S L_{2}(\mathbf{R}) \rightarrow P G L_{2}(\mathbf{R})$.

If $r \geq 3, \mathscr{V}_{0}$ is the subset of $P G L_{2}(\mathbf{R})^{S}$ consisting of all $\left(\gamma_{i}\right)_{i \in S}$ such that

(a) the points $\gamma_{i}(0) \in P_{\mathbf{R}}^{1}$ are distinct when $i$ runs through $S$; they cut the circle $P_{\mathbf{R}}^{1}$ into $r$ intervals and, for any $i \in S$, the points $\gamma_{i}(0), \gamma_{i+1}(0)$ are the ends of such an interval (here we regard $i$ as an integer modulo $r$ );

(b) for any $i \in S$, the set $\gamma_{i}((0, \delta))$ is contained in the interval with ends $\gamma_{i}(0), \gamma_{i+1}(0)$ (see (a)), provided that $\delta>0$ is sufficiently small.

Condition (b) can be reformulated as follows: for any $i$, the isomorphism $P_{\mathbf{R}}^{1} \rightarrow P_{\mathbf{R}}^{1}$ defined by $\gamma_{i}$ should be orientation preserving, where the first $P_{\mathbf{R}}^{1}$ is regarded as a circle oriented by $0 \mapsto 1 \mapsto \infty \mapsto 0$ and the second $P_{\mathbf{R}}^{1}$ is regarded as a circle oriented by $\gamma_{1}(0) \mapsto \gamma_{2}(0) \mapsto \cdots \mapsto \gamma_{r}(0) \mapsto \gamma_{1}(0)$.

The group $P G L_{2}(\mathbf{R})$ acts naturally on $\mathscr{V}_{0}$ by simultaneous left translation on each component. Let $\underline{\mathscr{V}}_{0}$ be the quotient space $P G L_{2}(\mathbf{R}) \backslash \mathscr{V}$.

Lemma 13.2. $\underline{\mathscr{V}}_{0}$ is a contractible real analytic manifold.

The case where $r=2$ is left to the reader. Assume now that $r \geq 3$. Let $F$ be the manifold consisting of sequences $p_{1}, p_{2}, \ldots, p_{r}$ of distinct points on $P_{\mathbf{R}}^{1}$ arranged according to the cyclic order of $S$ and taken modulo the action of $P G L_{2}(\mathbf{R})$. We may identify $F$ with an open convex set in $\mathbf{R}^{r-3}$. Now $\underline{\mathscr{V}}_{0}$ is naturally fibered over $F$ with fibres isomorphic to a product of $r$ copies of the identity component of the upper triangular subgroup of $P G L_{2}(\mathbf{R})$; hence, these fibres are isomorphic to $\mathbf{R} \times \mathbf{R}_{>0}$. The lemma follows.

13.3. Assume that we are given objects $V_{1}, V_{2}, \ldots, V_{r}$ of $\mathscr{O}_{\kappa}$. Let $Y=A \otimes\left(\bigotimes_{i=1}^{r} V_{i}\right)$ where $A$ is the coordinate algebra of $\mathscr{V}$ (as in 9.2, 9.3). By 12.12 , the $A$-module $Y / \Delta Y$ may be regarded as an algebraic vector bundle over $\mathscr{V}$ with a natural integrable connection. Hence its restriction to the real analytic submanifold $\mathscr{V}_{0}$ is a real analytic vector bundle $E$ with an integrable connection. Since $\mathscr{V}_{0}$ is contractible, the vector space $\mathscr{S}(E)$ of horizontal real analytic sections of $E$ gives a trivialization of $E$; namely, any fibre of $E$ is canonically isomorphic to $\mathscr{S}(E)$ as a vector space. (The isomorphism is given by restricting sections.)

We define

$$
\left\langle V_{1}, V_{2}, \ldots, V_{r}\right\rangle=\mathscr{S}(E) .
$$

This is a finite-dimensional $\mathbf{C}$-vector space. By its definition, it only depends 
on the cyclic order of $1,2, \ldots, r$ so that we have canonically

$$
\left\langle V_{1}, V_{2}, \ldots, V_{r}\right\rangle=\left\langle V_{2}, V_{3}, \ldots, V_{r}, V_{1}\right\rangle \text {. }
$$

For $r=2$, this coincides with our earlier definition of $\left\langle V_{1}, V_{2}\right\rangle$ (see 2.32) since $\left(\left(\begin{array}{cc}0 & -1 \\ 1 & 0\end{array}\right),\left(\begin{array}{ll}1 & 0 \\ 0 & 1\end{array}\right)\right)$ defines a point in $\underline{\mathscr{V}}_{0}$.

Next we note that, if $\left(\gamma_{i}\right)_{i \in S}$ defines a point of $\underline{\mathscr{V}}_{0}$ (with $r \geq 3$ ), then by omitting $\gamma_{1}$ we obtain a point $\left(\gamma_{i}\right)_{i \in S^{\prime}}$ (where $S^{\prime}=S-\{1\}$ ) which belongs to the manifold analogous to $\underline{\mathscr{V}}_{0}$ but is defined in terms of $S^{\prime}$ with the cyclic order induced from $S$. If we now take $V_{1}=V_{0}^{\kappa}$ then, using 17.18 , we obtain a canonical isomorphism

$$
\left\langle V_{2}, V_{3}, \ldots, V_{r}\right\rangle \cong\left\langle\mathbf{V}_{0}^{\kappa}, V_{2}, V_{3}, \ldots, V_{r}\right\rangle
$$

for any objects $V_{2}, V_{3}, \ldots, V_{r}$ in $\mathscr{O}_{\kappa}$.

13.4. Assume now that $r \geq 3$. Let $V_{1}, V_{2}, \ldots, V_{r-1}$ be objects of $\mathscr{O}_{\kappa}$. We apply to them the construction of $\S 4$ in the following special case: we take $S$ as above, $\mathcal{O}=\{r\}, \boldsymbol{\$}=\{1,2, \ldots, r-1\}$. We take $[r]=S$. We take $C=P^{1}$ with a system of charts $\gamma=\left(\gamma_{i}\right)_{i \in S}$ satisfying conditions (a),(b) in 13.1 .

The resulting $\widetilde{\mathbf{g}}$-module $T(W)^{\sharp}$ defined in terms of these data will be denoted ${ }_{\gamma}\left(V_{1} \dot{\otimes} V_{2} \dot{\otimes} \cdots \dot{\otimes} V_{r-1}\right)$. According to 7.9(a),

$$
{ }_{\gamma}\left(V_{1} \dot{\otimes} V_{2} \dot{\otimes} \cdots \dot{\otimes} V_{r-1}\right) \text { is an object of } \mathscr{O}_{\kappa} .
$$

Now let $V_{r}$ be another object of $\mathscr{O}_{\kappa}$.

We want to apply 7.10 with $W=V_{1} \otimes V_{2} \otimes \cdots \otimes V_{r-1}$ and $X=V_{r}$. The two vector spaces of coinvariants $(T(W) \otimes X)_{\tilde{\mathbf{g}}}$ and $(W \otimes X)_{\Gamma}$ appearing there are the same as $\left\langle_{\gamma}\left(V_{1} \dot{\otimes} V_{2} \dot{\otimes} \cdots \dot{\otimes} V_{r-1}\right), V_{r}\right\rangle$ and $\left(V_{1} \otimes V_{2} \otimes \cdots \otimes V_{r-1} \otimes V_{r}\right)_{\Delta_{C}}$ (coinvariants, with the notation $9.9(\mathrm{~b})$ ) respectively, where $\mathbf{C}$ is regarded as an $A$-algebra by evaluation at the point of $\mathscr{V}$ defined by $\gamma$. These vector spaces are finite dimensional by $2.32(\mathrm{~d})$ and 9.16 . Thus, 7.10 is applicable and gives us a canonical isomorphism between these two vector spaces:

$$
\left\langle_{\gamma}\left(V_{1} \dot{\otimes} V_{2} \dot{\otimes} \cdots \dot{\otimes} V_{r-1}\right), V_{r} \cong \cong\left(V_{1} \otimes V_{2} \otimes \cdots \otimes V_{r-1} \otimes V_{r}\right)_{\Delta_{\mathbf{c}}}\right.
$$

By definition, $\left(V_{1} \otimes V_{2} \otimes \cdots \otimes V_{r-1} \otimes V_{r}\right)_{\Delta_{C}}$ is the fibre of the vector bundle $E$ (defined in 13.3) at the point of $\underline{\mathscr{V}}_{0}$ defined by $\gamma$. As we have seen earlier, that fibre is canonically isomorphic to $\mathscr{S}(E)=\left\langle V_{1}, V_{2}, \ldots, V_{r-1}, V_{r}\right\rangle$. We therefore obtain a canonical isomorphism of $\mathbf{C}$-vector spaces

$$
\left\langle{ }_{\gamma}\left(V_{1} \dot{\otimes} V_{2} \dot{\otimes} \cdots \dot{\otimes} V_{r-1}\right), V_{r}\right\rangle \cong\left\langle V_{1}, V_{2}, \ldots, V_{r-1}, V_{r}\right\rangle
$$

Using 2.32(c), this can be also expressed as an isomorphism

(b) $\operatorname{Hom}_{\mathscr{O}_{\kappa}}\left({ }_{\gamma}\left(V_{1} \dot{\otimes} V_{2} \dot{\otimes} \cdots \dot{\otimes} V_{r-1}\right), D\left(V_{r}\right)\right) \cong \operatorname{Hom}_{\mathbf{C}}\left(\left\langle V_{1}, V_{2}, \ldots, V_{r-1}, V_{r}\right\rangle, \mathbf{C}\right)$.

The vector space in the right-hand side of $(b)$ is independent of $\gamma$ (as above), and hence so is the one in the left-hand side. It follows that the objects ${ }_{\gamma}\left(V_{1} \dot{\otimes} V_{2} \dot{\otimes} \cdots \dot{\otimes} V_{r-1}\right) \in \mathscr{O}_{\kappa}$ are canonically isomorphic to each other (when $\gamma$ 
varies) so that they can be considered as a single object $V_{1} \dot{\otimes} V_{2} \dot{\otimes} \cdots \dot{\otimes} V_{r-1} \in \mathscr{O}_{\kappa}$ defined up to unique isomorphism and independent of $\gamma$.

We can rewrite the earlier isomorphisms as follows:

$$
\left\langle V_{1} \dot{\otimes} V_{2} \dot{\otimes} \cdots \dot{\otimes} V_{r-1}, V_{r}\right) \cong\left\langle V_{1}, V_{2}, \ldots, V_{r-1}, V_{r}\right\rangle
$$

(d) $\operatorname{Hom}_{\mathbf{C}}\left(\left\langle V_{1}, V_{2}, \ldots, V_{r-1}, V_{r}\right\rangle, \mathbf{C}\right) \cong \operatorname{Hom}_{\mathscr{O}_{x}}\left(V_{1} \dot{\otimes} V_{2} \dot{\otimes} \cdots \dot{\otimes} V_{r-1}, D\left(V_{r}\right)\right)$.

13.5. The previous discussion is applicable in particular for $r=3$. In this case we shall take $S=\{1, \infty, 0\}$ with the cyclic order $1<\infty<0<1$ and $\odot=\{0\}, \boldsymbol{\phi}=\{1, \infty\},[0]=S$.

Let $C=P^{1}$ with the following system of charts:

$$
\gamma_{1}=\left(\begin{array}{cc}
1 & -1 \\
1 & 0
\end{array}\right), \quad \gamma_{\infty}=\left(\begin{array}{cc}
0 & 1 \\
-1 & 1
\end{array}\right), \quad \gamma_{0}=\left(\begin{array}{ll}
1 & 0 \\
0 & 1
\end{array}\right) .
$$

(These charts are characterized as follows: $\gamma_{1}$ takes $1, \infty, 0 \in P_{\mathbf{R}}^{1}$ to $0,1, \infty$ respectively; $\gamma_{\infty}$ takes $\infty, 0,1 \in P_{\mathbf{R}}^{1}$ to $0,1, \infty$ respectively; $\gamma_{0}$ takes 0,1 , $\infty \in P_{\mathbf{R}}^{1}$ to $0,1, \infty$ respectively.) This system of charts satisfies the conditions (a),(b) in 13.1 (thus, $\mathscr{V}_{0}$ has in this case a distinguished point). Hence in this case, given two objects $V, V^{\prime}$ of $\mathscr{O}_{\kappa}$, we can define $V \dot{\otimes} V^{\prime} \in \mathscr{O}_{\kappa}$ with reference to this system of charts. $\left(V, V^{\prime}\right.$ are indexed by $1, \infty$ in $S$ respectively.) Specializing 13.4(c),(d) to $r=3$, we obtain

$$
\operatorname{Hom}_{\mathbf{C}}\left(\left\langle V, V^{\prime}, V^{\prime \prime}\right\rangle, \mathbf{C}\right) \cong \operatorname{Hom}_{\mathscr{O}_{\kappa}}\left(V \dot{\otimes} V^{\prime}, D\left(V^{\prime \prime}\right)\right) .
$$

13.6. For any $V$ in $\mathscr{O}_{\kappa}$, we have unique isomorphisms in $\mathscr{O}_{\kappa}$ :

$$
V \cong \mathbf{V}_{0}^{\kappa} \dot{\otimes} V, \quad V \cong V \dot{\otimes} \mathbf{V}_{0}^{\kappa}
$$

such that for any $V^{\prime}$ in $\mathscr{O}_{\kappa}$ the induced isomorphisms $\left\langle V, V^{\prime}\right\rangle \cong\left\langle\mathbf{V}_{0}^{\kappa} \dot{\otimes} V, V^{\prime}\right\rangle$ (resp. $\left.\left\langle V, V^{\prime}\right\rangle \cong\left\langle V \dot{\otimes} \mathbf{V}_{0}^{\kappa}, V^{\prime}\right\rangle\right)$ are obtained by the composition

$$
\left\langle V, V^{\prime}\right\rangle \cong\left\langle\mathbf{V}_{0}^{\kappa}, V, V^{\prime}\right\rangle \cong\left\langle\mathbf{V}_{0}^{\kappa} \dot{\otimes} V, V^{\prime}\right\rangle
$$

(resp. by the composition

$$
\left.\left\langle V, V^{\prime}\right\rangle=\left\langle V^{\prime}, V\right\rangle \cong\left\langle\mathbf{v}_{0}^{\kappa}, V^{\prime}, V\right\rangle=\left\langle V, \mathbf{v}_{0}^{\kappa}, V^{\prime}\right\rangle \cong\left\langle V \dot{\otimes} \mathbf{V}_{0}^{\kappa}, V^{\prime}\right\rangle\right) ;
$$

see $13.3(\mathrm{c})$ and $13.5(\mathrm{~b})$.

\section{COMMUTATIVITY ISOMORPHISM}

In this section we will show that, given two objects $V, V^{\prime}$ of $\mathscr{O}_{\kappa}$, the objects $V \dot{\otimes} V^{\prime}$ and $V^{\prime} \dot{\otimes} V$ of $\mathscr{O}_{\kappa}$ are isomorphic. In fact, we will construct two isomorphisms, one for each choice of a square root of -1 in $\mathbf{C}$. 
14.1. We shall need some commutation formulas for Sugawara operators. Let $V$ be an object in $\mathscr{O}_{\kappa}$. The operator $L_{0}: V \rightarrow V$ is locally finite; hence, for any $a \in \mathbf{C}$, the exponential $e^{a L_{0}}: V \rightarrow V$ is well defined. The operator $L_{1}: V \rightarrow V$ is locally nilpotent (it maps ${ }_{\lambda} V$ into ${ }_{\lambda-1} V$ ); hence, the exponential $e^{b L_{1}}: V \rightarrow V$ is well defined for any $b \in \mathbf{C}$. Let $f(\epsilon) \in \mathbf{C}((\epsilon))$ and let $c \in \mathbf{g}$. The following identities of endomorphisms of $V$ follow easily from 1.14(b), 1.14(c):

$$
\begin{gathered}
(f(\epsilon) c) e^{a L_{0}}=e^{a L_{0}}\left(f\left(e^{a} \epsilon\right) c\right) \\
(f(\epsilon) c) e^{b L_{1}}=e^{b L_{1}}\left(f\left(\frac{\epsilon}{1-b \epsilon}\right) c\right) \\
e^{b L_{1}} e^{a L_{0}}=e^{a L_{0}} e^{e^{a} b L_{1}}
\end{gathered}
$$

Hence, if we set

$$
\tau=e^{\sqrt{-1} \pi L_{0}} e^{L_{1}}=e^{-L_{1}} e^{\sqrt{-1} \pi L_{0}}: V \rightarrow V
$$

and

$$
\bar{\tau}=e^{-\sqrt{-1} \pi L_{0}} e^{L_{1}}=e^{-L_{1}} e^{-\sqrt{-1} \pi L_{0}}=\tau^{-1}: V \rightarrow V
$$

(where $\sqrt{-1}$ is a square root of -1 in $\mathbf{C}$ ), then

(d)

$$
(f(\epsilon) c) \tau=\tau\left(f\left(\frac{\epsilon}{\epsilon-1}\right) c\right), \quad(f(\epsilon) c) \bar{\tau}=\bar{\tau}\left(f\left(\frac{\epsilon}{\epsilon-1}\right) c\right), \quad \tau \bar{\tau}=\bar{\tau} \tau=1
$$

as endomorphisms of $V$. We have

$$
\tau^{2}=e^{2 \sqrt{-1} \pi L_{0}}, \quad \bar{\tau}^{2}=e^{-2 \sqrt{-1} \pi L_{0}} .
$$

From (a),(e), we see that $\tau^{2}$ and $\bar{\tau}^{2}$ commute with the $\widetilde{\mathbf{g}}$-action on $V$.

14.2. Let $V, V^{\prime}, V^{\prime \prime}$ be three objects of $\mathscr{O}_{\kappa}$. Let

$$
P, \bar{P}: V \otimes V^{\prime} \otimes V^{\prime \prime} \rightarrow V^{\prime} \otimes V \otimes V^{\prime \prime}
$$

be the linear maps given by

$$
P(x \otimes y \otimes z)=\tau y \otimes \tau x \otimes \bar{\tau} z, \quad \bar{P}(x \otimes y \otimes z)=\bar{\tau} y \otimes \bar{\tau} x \otimes \tau z
$$

where $\tau, \bar{\tau}$ are as in 14.1 .

Lemma 14.3. For any vectors $x, y, z$ in $V, V^{\prime}, V^{\prime \prime}$ and any rational function $f(\epsilon) \in \mathbf{C}\left[\epsilon, \epsilon^{-1},\left(1-\epsilon^{-1}\right)\right]$, we have $\tau y \otimes \tau\left(f\left(\frac{\epsilon-1}{\epsilon}\right) c x\right) \otimes \bar{\tau} z+\tau\left(f\left(\frac{1}{1-\epsilon}\right) c y\right) \otimes \tau x \otimes \bar{\tau} z+\tau y \otimes \tau x \otimes \bar{\tau}(f(\epsilon) c z)$

$$
\begin{aligned}
= & g\left(\frac{\epsilon-1}{\epsilon}\right) c \tau y \otimes \tau x \otimes \bar{\tau} z+\tau y \otimes g\left(\frac{1}{1-\epsilon}\right) c \tau x \otimes \bar{\tau} z \\
& +\tau y \otimes \tau x \otimes g(\epsilon) c \bar{\tau} z
\end{aligned}
$$

where $g(\epsilon)=f\left(\frac{\epsilon}{\epsilon-1}\right)$. 
It is enough to verify

$$
\begin{aligned}
& g(\epsilon) c \bar{\tau}=\bar{\tau} g\left(\frac{\epsilon}{\epsilon-1}\right) c=\bar{\tau} f(\epsilon) c \\
& g\left(\frac{1}{1-\epsilon}\right) c \tau=\tau g(1-\epsilon) c=\tau f\left(\frac{\epsilon-1}{\epsilon}\right) c, \\
& g\left(\frac{\epsilon-1}{\epsilon}\right) c \tau=\tau g\left(\epsilon^{-1}\right) c=\tau f\left(\frac{1}{1-\epsilon}\right) c .
\end{aligned}
$$

These identities follow from 14.1(c) and the definition of $g$.

14.4. We regard $V \otimes V^{\prime} \otimes V^{\prime \prime}$ and $V^{\prime} \otimes V \otimes V^{\prime \prime}$ as $\Delta_{\mathrm{C}}$-modules as in 9.11, where $\mathbf{C}$ is an $A$-algebra by evaluation at the point of $\mathscr{V}$ defined by $13.5(\mathrm{a})$. (Note that $V, V^{\prime}, V^{\prime \prime}$ are indexed by $1, \infty, 0$ in the first tensor product and $V^{\prime}, V, V^{\prime \prime}$ are indexed by $1, \infty, 0$ in the second tensor product.)

Proposition 14.5. $P$ and $\bar{P}$ map the subspace $\Delta_{\mathbf{C}}\left(V \otimes V^{\prime} \otimes V^{\prime \prime}\right)$ of $\left(V \otimes V^{\prime} \otimes V^{\prime \prime}\right)$ into the subspace $\Delta_{\mathbf{C}}\left(V^{\prime} \otimes V \otimes V^{\prime \prime}\right)$ of $\left(V^{\prime} \otimes V \otimes V^{\prime \prime}\right)$ and induce isomorphisms on coinvariants

$$
\left(V \otimes V^{\prime} \otimes V^{\prime \prime}\right)_{\Delta_{\mathbf{C}}} \cong\left(V^{\prime} \otimes V \otimes V^{\prime \prime}\right)_{\Delta_{\mathbf{C}}}
$$

Moreover, the compositions

$$
\begin{aligned}
& \left(V \otimes V^{\prime} \otimes V^{\prime \prime}\right)_{\Delta_{\mathbf{C}}} \stackrel{P}{\longrightarrow}\left(V^{\prime} \otimes V \otimes V^{\prime \prime}\right)_{\Delta_{\mathbf{C}}} \stackrel{\bar{P}}{\longrightarrow}\left(V \otimes V^{\prime} \otimes V^{\prime \prime}\right)_{\Delta_{\mathbf{C}}} \\
& \left(V \otimes V^{\prime} \otimes V^{\prime \prime}\right)_{\Delta_{\mathbf{C}}} \stackrel{\bar{P}}{\longrightarrow}\left(V^{\prime} \otimes V \otimes V^{\prime \prime}\right)_{\Delta_{\mathbf{C}}} \stackrel{P}{\longrightarrow}\left(V \otimes V^{\prime} \otimes V^{\prime \prime}\right)_{\Delta_{\mathbf{C}}}
\end{aligned}
$$

are the identity maps.

The statement about $P$ follows immediately from the previous lemma; the statement about $\bar{P}$ is proved in exactly the same way. The last statement follows from $\tau \bar{\tau}=\bar{\tau} \tau=1$.

14.6. Let $V, V^{\prime}$ be two objects of $\mathscr{O}_{\kappa}$. We want to define an isomorphism $D\left(V^{\prime} \dot{\otimes} V\right) \cong D\left(V \dot{\otimes} V^{\prime}\right)\left(\right.$ in $\left.\mathscr{O}_{\kappa}\right)$.

By definition, $D\left(V \dot{\otimes} V^{\prime}\right)=T^{\prime}(W)$ and $D\left(V \dot{\otimes} V^{\prime}\right)=T^{\prime}\left(W^{\prime}\right)$ where $W=V \otimes V^{\prime}, W^{\prime}=V^{\prime} \otimes V$, and $T^{\prime}(W), T^{\prime}\left(W^{\prime}\right)$ are constructed as in $\S 6$ in terms of the filtrations $\left(G_{N} W\right)$ and $\left(G_{N} W^{\prime}\right)$ of $W, W^{\prime}$.

We define an isomorphism $P: W \cong W^{\prime}$ by $P(x \otimes y)=\tau y \otimes \tau x$ where $\tau$ is as in 14.1. Let $Z=\operatorname{Hom}(W, \mathbf{C}), Z^{N}, Z^{\infty}$ be as in 6.2 , and let $Z^{\prime}=$ $\operatorname{Hom}\left(W^{\prime}, \mathbf{C}\right), Z^{\prime N}, Z^{\prime \infty}$ be the analogous spaces defined in terms of $W^{\prime}$.P defines an isomorphism $Z^{\prime} \cong Z$ (the transpose of $P: W \cong W^{\prime}$ ); we denote this again by $P$.

We show that $P\left(Z^{\prime N}\right)=Z^{N}$. Since $Z^{N}, Z^{\prime N}$ are the annihilators of $G_{N} W$, $G_{N} W^{\prime}$, it suffices to show that $P: W \cong W^{\prime}$ maps $G_{N} W$ onto $G_{N} W^{\prime}$.

Let $f(\epsilon) \in \epsilon \mathbf{C}\left[\epsilon,(1-\epsilon)^{-1}\right]$ and let $c \in \mathbf{g}$. Then $(f(\epsilon) c)$ may be regarded as an element of $G_{1}$. It acts on $W$ as $f(\epsilon) c(x \otimes y)=f\left(\frac{\epsilon-1}{\epsilon}\right) c x \otimes y+x \otimes f\left(\frac{1}{1-\epsilon}\right) c y$ 
and on $W^{\prime}$ by $f(\epsilon) c(y \otimes x)=f\left(\frac{\epsilon-1}{\epsilon}\right) c y \otimes x+y \otimes f\left(\frac{1}{1-\epsilon}\right) c x$. Let $g(\epsilon)=$ $f\left(\frac{\epsilon}{\epsilon-1}\right) \in \epsilon \mathbf{C}\left[\epsilon,(1-\epsilon)^{-1}\right]$. Using 14.3(a), we see that

$$
P(f(\epsilon) c(x \otimes y))=(g(\epsilon) c)(P(x \otimes y)) .
$$

Applying this $N$ times we see that $P\left(G_{N} W\right)=G_{N} W^{\prime}$. Hence, $P\left(Z^{\prime N}\right)=Z^{N}$ for all $N \geq 1$. It follows that $P\left(Z^{\prime \infty}\right)=Z^{\infty}$. Recall from 6.3 that $Z^{\prime \infty}, Z^{\infty}$ are naturally smooth $\widetilde{\mathbf{g}}$-modules (actually in $\mathscr{O}_{\kappa}$, by $7.9(\mathrm{a})$ ). Hence the operator $\tau$ is well defined on $Z^{\prime \infty}, Z^{\infty}$.

Using the definitions, we see that $P: Z^{\prime \infty} \rightarrow Z^{\infty}$ satisfies

$$
P\left((f(\epsilon) c) z^{\prime}\right)=\left(f\left(\frac{\epsilon}{\epsilon-1}\right) c\right) P\left(z^{\prime}\right)
$$

for all $f(\epsilon) \in \mathbf{C}\left[\epsilon, \epsilon^{-1},\left(1-\epsilon^{-1}\right)\right] \subset \mathbf{C}((\epsilon))$ and all $c \in \mathbf{g}$ and $z^{\prime} \in Z^{\prime \infty}$.

The composition

$$
Z^{\prime \infty} \stackrel{\bar{\tau}}{\longrightarrow} Z^{\prime \infty} \stackrel{P}{\longrightarrow} Z^{\infty}
$$

satisfies then

$$
P \bar{\tau}\left((f(\epsilon) c) z^{\prime}\right)=(f(\epsilon) c) P \bar{\tau}\left(z^{\prime}\right)
$$

for all $f, c, z^{\prime}$ as above. Hence, it is an isomorphism of $\widetilde{\mathbf{g}}$-modules. Thus, we have constructed an isomorphism $\widetilde{\mathbf{g}}$-modules $P \bar{\tau}: T^{\prime}\left(W^{\prime}\right) \cong T^{\prime}(W)$ or, equivalently, (see 7.9) an isomorphism of $\widetilde{\mathbf{g}}$-modules

$$
D\left(V^{\prime} \dot{\otimes} V\right) \cong D\left(V \dot{\otimes} V^{\prime}\right) .
$$

We want to relate (b) to the isomorphism of 14.5 .

Taking the transpose of the isomorphism $\left\langle V \dot{\otimes} V^{\prime}, V^{\prime \prime}\right\rangle \cong\left\langle V, V^{\prime}, V^{\prime \prime}\right\rangle$ of 13.5, we obtain an isomorphism

$$
\operatorname{Hom}_{\mathbf{C}}\left(\left\langle V, V^{\prime}, V^{\prime \prime}\right\rangle, \mathbf{C}\right) \cong \operatorname{Hom}_{\mathscr{O}_{\kappa}}\left(V^{\prime \prime}, D\left(V \dot{\otimes} V^{\prime}\right)\right) .
$$

Similarly, by interchanging $V, V^{\prime}$, we have an isomorphism

$$
\operatorname{Hom}_{\mathbf{C}}\left(\left\langle V^{\prime}, V, V^{\prime \prime}\right\rangle, \mathbf{C}\right) \cong \operatorname{Hom}_{\mathscr{O}_{\kappa}}\left(V^{\prime \prime}, D\left(V^{\prime} \dot{\otimes} V\right)\right) .
$$

We consider the diagram

(c)

$$
\operatorname{Hom}_{\mathbf{C}}\left(\left\langle V^{\prime}, V, V^{\prime \prime}\right\rangle, \mathbf{C}\right) \longrightarrow \operatorname{Hom}_{\mathscr{\theta}_{\kappa}}\left(V^{\prime \prime}, D\left(V^{\prime} \dot{\otimes} V\right)\right)
$$

where the horizontal maps are the isomorphisms above, the left vertical map is the transpose of $P:\left\langle V, V^{\prime}, V^{\prime \prime}\right\rangle \cong\left\langle V^{\prime}, V, V^{\prime \prime}\right\rangle$ (see 14.5), and the right vertical map is induced by (b).

Using the definitions, we can check that the diagram (c) is commutative.

Taking the transpose of (b) we obtain an isomorphism

$$
\mathscr{P}: V \dot{\otimes} V^{\prime} \cong V^{\prime} \dot{\otimes} V \quad \text { in } \mathscr{O}_{\kappa} .
$$


Note that $\mathscr{P}$ was defined in terms of $\tau$ and maps derived from it (like $P$ ); we could have equally well used $\bar{\tau}, \bar{P}$ instead of $\tau, P$; we then get an isomorphism

$$
\overline{\mathscr{P}}: V \dot{\otimes} V^{\prime} \cong V^{\prime} \dot{\otimes} V \quad \text { in } \mathscr{O}_{\kappa} .
$$

Using 14.1(d), we see that the compositions

$$
V \dot{\otimes} V^{\prime} \stackrel{\mathscr{P}}{\longrightarrow} V^{\prime} \dot{\otimes} V \stackrel{\overline{\mathscr{P}}}{\longrightarrow} V \dot{\otimes} V^{\prime}
$$

and

$$
V \dot{\otimes} V^{\prime} \stackrel{\overline{\mathscr{P}}}{\longrightarrow} V^{\prime} \dot{\otimes} V \stackrel{\mathscr{P}}{\longrightarrow} V \dot{\otimes} V^{\prime}
$$

are the identity.

\section{Degeneration OF QUADRICS (I)}

In this section we begin the construction of the associativity isomorphism for $\dot{\otimes}$, which will be completed in $\S 18$. This involves studying the way in which coinvariants behave when we approach a "boundary point" of $\mathscr{V}$ (for $|S|=$ 4). Geometrically, this involves looking at a family of nonsingular quadrics degenerating to the union of two lines. The main result of this section will be the construction of a family of maps $\Phi_{n}$ (see 15.25(a)), one for each integer $n \geq 1$, which relate two spaces of coinvariants: one on the degenerate quadric, the other on the nondegenerate ones.

15.1. We assume that $S$ is a finite set such that $|S|=4$, with a given cyclic order. We shall write the elements of $S$ as the vertices $1,2,3,4$ of a square

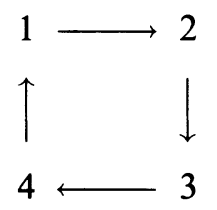

where $a \rightarrow b$ stands for $a<b$ in the cyclic order. (Thus, $1<2<3<4<1$.)

We will make some constructions which involve an additional choice; namely, we single out the two horizontal sides of the square. (A completely similar discussion will apply if we single out the two vertical sides.)

We assume given four objects

of $\mathscr{O}_{\kappa}$, indexed as shown by $S$.

$$
\begin{array}{ll}
V_{1} & V_{2} \\
V_{4} & V_{3}
\end{array}
$$

15.2. Let

$$
D=\{t \in \mathbf{C} \mid t \neq 1\}, \quad D^{\prime}=\left\{(t, p, q) \in \mathbf{C}^{3} \mid p q=t, t \neq 1, p \neq 1, q \neq 1\right\} .
$$

The variables $p, q$ should be regarded as being associated with the upper horizontal side (12) (resp. the lower horizontal side (34) of the square in 15.1.

Let $\pi: D^{\prime} \rightarrow D$ be the first projection. For each $t \in D$, the fibre $\pi^{-1}(t)$ may be thought of as the quadric $K_{t}$ with equation $p q=t$ in $P^{1} \times P^{1}$, from which four distinct points have been removed, namely,

$$
\begin{array}{cc}
(1, t) & (\infty, 0) \\
(0, \infty) & (t, 1)
\end{array}
$$


(indexed as shown by the elements of $S$ ).

This quadric is nonsingular for $t \neq 0$ and degenerates to a union of two lines for $t=0$; but the four special points above are always smooth points of it.

15.3. Corresponding to each of these four points, we have a canonical isomorphism of $P^{1}$ onto the irreducible component of the quadric containing that point (and taking 0 to that point). This maps $z \in P^{1}$ to the point shown in the corresponding vertex of the square:

$$
\begin{gathered}
\left(\frac{1}{1-z}, t(1-z)\right) \quad\left(\frac{z-1}{z}, \frac{t z}{z-1}\right) \\
\left(\frac{t z}{z-1}, \frac{z-1}{z}\right) \quad\left(t(1-z), \frac{1}{1-z}\right)
\end{gathered}
$$

15.4. Let

$$
\begin{aligned}
& A_{\infty}=\mathbf{C}[D]=\mathbf{C}\left[t,(t-1)^{-1}\right], \\
& A_{\infty}^{\prime}=\mathbf{C}\left[D^{\prime}\right]=\mathbf{C}\left[t, p, q,(t-1)^{-1},(p-1)^{-1},(q-1)^{-1}\right] /(p q-t) .
\end{aligned}
$$

For any integer $n \geq 1$ we define $A_{n}=A_{\infty} /\left(t^{n}\right)=\mathbf{C}\left[t,(t-1)^{-1}\right] /\left(t^{n}\right)=$ $\mathbf{C}[t] /\left(t^{n}\right)$ and $A_{n}^{\prime}=A_{\infty}^{\prime} /\left(t^{n}\right)=A_{n}\left[p, q,(p-1)^{-1},(q-1)^{-1}\right] /(p q-t)$.

For each $i \in S$, we have an $A_{\infty}$-algebra homomorphism

$$
A_{\infty}^{\prime} \rightarrow A_{\infty}((\epsilon)) \quad\left(f \mapsto{ }^{i} f\right) .
$$

It is defined as follows. For a fixed $t$, we restrict $f \in A_{\infty}^{\prime}$ to $\pi^{-1}(t)$. We may regard this restriction as a rational function on $K_{t}$ and, via the correspondence shown in $\mathbf{1 5 . 3}$ for the $i$-vertex of our square, as a rational function on $P^{1}$. We take the power series expansion of that rational function at 0 . That power series may be regarded as a power series with coefficients in $A_{\infty}$, and this is, by definition, ${ }^{i} f$.

The homomorphism (a) induces for any integer $n \geq 1$ a homomorphism

$$
A_{n}^{\prime} \rightarrow A_{n}((\epsilon)) \quad\left(f \mapsto^{i} f\right) .
$$

15.5. Under the correspondence

$$
f \mapsto{ }^{4} f{ }^{3} f
$$

we have, for example,

$$
p \mapsto \begin{array}{cc}
\frac{1}{1-\epsilon} & \frac{\epsilon-1}{\epsilon} \\
\frac{t \epsilon}{1-\epsilon} & t(1-\epsilon)
\end{array} \quad q \mapsto \begin{array}{cc}
t(1-\epsilon) & \frac{t \epsilon}{\epsilon-1} \\
\frac{\epsilon-1}{\epsilon} & \frac{1}{1-\epsilon}
\end{array}
$$

for $1 \leq n \leq \infty$.

15.6. Consider the four elements $\begin{aligned} & f_{1} f_{2} \\ & f_{4} f_{3}\end{aligned}$ of $A_{n}^{\prime}$ given by

$$
\begin{array}{cc}
p(p-1)^{-1} & 1-p \\
1-q & q(q-1)^{-1}
\end{array}
$$

The image of $f_{i}$ under $15.5(\mathrm{a})$ has the following property: its $i$ th coordinate is $\epsilon^{-1}$ and its other three coordinates are in $A_{n}[[\epsilon]]$. 
Lemma 15.7. The functions

$$
f_{i}^{k} \quad(s \in S ; k \geq 1) \text { and } 1
$$

form a basis of $A_{n}^{\prime}$ as an $A_{n}$-module.

It is enough to prove this for $n=\infty$. In that case we argue just as in the proof of 9.6, with only one difference: in the earlier proof one step was the fact that a regular function on $P^{1}$ must be constant; we must now use the more general fact that a regular function on a possibly reducible, connected projective curve is constant.

15.8. Let $\Delta_{4, n}$ be the Lie algebra $A_{n}^{\prime} \otimes \mathbf{g}$ with bracket $\left[f c, f^{\prime} c^{\prime}\right]=f f^{\prime}\left[c, c^{\prime}\right]$ for $f, f^{\prime} \in A_{n}^{\prime}$ and $c, c^{\prime} \in \mathbf{g}$.

Lemma 15.9. If $1 \leq n \leq \infty$, there is a unique homomorphism of $A_{n}$-Lie algebras $\Delta_{4, n} \rightarrow \widehat{\mathbf{g}}_{A_{n}}^{S}$ such that

$$
f c \mapsto{ }^{4} f c{ }^{2} f c
$$

for all $f \in A_{n}^{\prime}$ and $c \in \mathbf{g}$.

Let $f, f^{\prime} \in A_{n}^{\prime}$ and let

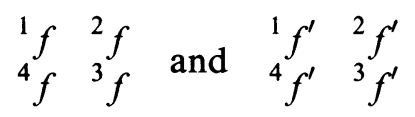

be their images under the map 15.5(a). It is enough to prove that the following element of $A_{n}$ is zero:

$$
\sum_{i=1}^{4} \operatorname{Res}_{\epsilon=0}\left({ }^{i} f^{\prime} d\left({ }^{i} f\right)\right)
$$

(here $d$ is with respect to $\epsilon$ ). We may assume that $n=\infty$ (the general case follows from this). We may specialize to a fixed value of $t \neq 1$. Then $f, f^{\prime}$ can be regarded as functions on the (possibly singular) quadric $K_{t}$ which are regular outside the four marked smooth points of that quadric; we see that (a) is just the sum of residues of the differential form $f^{\prime} d(f)$ on the quadric at the four marked points. This is zero by the residue theorem. The lemma is proved.

15.10. Let $Y_{n}=A_{n} \otimes\left(\otimes_{i=1}^{4} V_{i}\right)$. This is a $\widehat{\mathbf{g}}_{A_{n}}^{S}$-module as in 1.13 and, hence, a $\Delta_{4, n}$-module, via 15.9 .

15.11. In the case where, for each $s, V_{s}$ is the generalized Weyl module corresponding to the nil-module $\mathscr{N}_{s}$ of $\mathbf{C}[\epsilon] \otimes b g$, we have an isomorphism

$$
\left(A_{n} \otimes\left(\bigotimes_{s} \mathscr{N}_{s}\right)\right)_{\mathbf{g}} \cong Y_{n} / \Delta_{4, n} Y_{n}
$$

induced on coinvariants by the obvious inclusion $B_{n} \otimes\left(\bigotimes_{s} \mathscr{N}_{s}\right) \subset Y_{n}$. Here, $1 \leq n \leq \infty$.

The proof is exactly the same as that of 9.15 . (It uses 15.7 instead of 9.6.) 
15.12. Returning to the general case, we deduce from 15.11 , exactly as in 9.16 , that

(a) If $1 \leq n \leq \infty$, then $Y_{n} / \Delta_{4, n} Y_{n}$ is a finitely generated $A_{n}$ module.

15.13. We shall need a simpler version of the earlier results in this section (in which 4 is replaced by 2 ).

Let us now assume that instead of $S$ we are given the set $\odot$ with two elements 12,34 corresponding to the two horizontal edges of our square and that we are given two objects $X_{12}, X_{34}$ of $\mathscr{O}_{\kappa}$.

Let

$$
B_{\infty}=\mathbf{C}[t], \quad B_{\infty}^{\prime}=\mathbf{C}[t, p, q,] /(p q-t) .
$$

For any integer $n \geq 1$ we define $B_{n}=B_{\infty} /\left(t^{n}\right)=\mathrm{C}[t] /\left(t^{n}\right)$ and $B_{n}^{\prime}=$ $B_{\infty}^{\prime} /\left(t^{n}\right)=B_{n}[p, q] /(p q-t)$.

For $1 \leq n \leq \infty$ we have a $B_{n}$-algebra homomorphism

$$
B_{n}^{\prime} \rightarrow B_{n}((\epsilon)) \oplus B_{n}((\epsilon))
$$

given by

$$
p \mapsto\left(-\epsilon^{-1},-t \epsilon\right), \quad q \mapsto\left(-t \epsilon,-\epsilon^{-1}\right) .
$$

Taking tensor product with the identity map of $\mathbf{g}$, we obtain a $B_{n}$-linear $\operatorname{map} \Delta_{2, n} \rightarrow\left(B_{n}\left[\epsilon, \epsilon^{-1}\right] \otimes \mathbf{g}\right) \oplus\left(B_{n}\left[\epsilon, \epsilon^{-1}\right] \otimes \mathbf{g}\right)$ where

$$
\Delta_{2, n}=\left(B_{n}[p, q] /(p q-t)\right) \otimes \mathbf{g} .
$$

Composing this with the obvious inclusion

$$
\left(B_{n}\left[\epsilon, \epsilon^{-1}\right] \otimes \mathbf{g}\right) \oplus\left(B_{n}\left[\epsilon, \epsilon^{-1}\right] \otimes \mathbf{g}\right) \subset \widetilde{\mathbf{g}}_{B_{n}}^{\odot},
$$

we obtain a $B_{n}$-linear map

$$
\Delta_{2, n} \rightarrow \widetilde{\mathbf{g}}_{B_{n}}^{\odot}
$$

Now $\Delta_{2, n}$ is a $B_{n}$-Lie algebra with bracket $\left[f c, f^{\prime} c^{\prime}\right]=f f^{\prime}\left[c, c^{\prime}\right]$ for $f, f^{\prime} \in B_{n}[p, q] /(p q-t)$ and $c, c^{\prime} \in \mathbf{g}$.

Note that (b) is a Lie algebra homomorphism. (This can be verified directly or can be proved in the same way as the corresponding result for $\Delta_{4, n}$.)

We may regard $B_{n} \otimes\left(X_{12} \otimes X_{34}\right)$ as a $\widehat{\mathbf{g}}_{B_{n}}^{\odot}$-module as in 1.13 and as a $\Delta_{2, n^{-}}$ module, via (b).

Lemma 15.14. If $1 \leq n \leq \infty$, then the $B_{n}$-module $\left(B_{n} \otimes\left(X_{12} \otimes X_{34}\right)\right)_{\Delta_{2, n}}$ is finitely generated.

This can be proved as for $\Delta_{4, n}$.

15.15. By the right exactness of tensor products we have

$$
B_{n} \otimes_{B_{\infty}}\left(B_{\infty} \otimes X_{12} \otimes X_{34}\right)_{\Delta_{2, \infty}} \cong\left(B_{n} \otimes X_{12} \otimes X_{34}\right)_{\Delta_{2, n}} .
$$


15.16. We now take $X_{12}=V_{1} \dot{\otimes} V_{2}, X_{34}=V_{3} \dot{\otimes} V_{4}$; from 15.14 we obtain: If $1 \leq n \leq \infty$, the $B_{n}$-module $\left(B_{n} \otimes\left(V_{1} \dot{\otimes} V_{2}\right) \otimes\left(V_{3} \dot{\otimes} V_{4}\right)\right)_{\Delta_{2, n}}$ is finitely generated.

15.17. We consider the setup in 4.2. We start with the finite set $S^{\prime}=S \cup \varnothing$ with $S, \mathcal{Q}$ as above. (The role of $\uparrow, S$ in 4.2 is now played by $S, S^{\prime}$.)

Let [12] $=\{1,2,12\}$ and [34] $=\{3,4,34\}$. We take $C$ to be the projective curve $P^{1} \cup P^{1}$ (disjoint union); the first $P^{1}$ is denoted $C_{12}$, the second one is denoted $C_{34}$. We specify the following points on $C:$ on $C_{12}$, we specify $p_{1}=1, p_{2}=\infty, p_{12}=0$; on $C_{34}$, we specify $p_{3}=1, p_{4}=\infty, p_{34}=0$. We also specify the following system of charts: for $C_{12}$, we take

$$
\begin{aligned}
& \gamma_{1}: P^{1} \cong C_{12} \text { given by } z \mapsto \frac{1}{1-z}, \\
& \gamma_{2}: P^{1} \cong C_{12} \text { given by } z \mapsto \frac{z-1}{z}, \\
& \gamma_{12}: P^{1} \cong C_{12} \text { given by } z \mapsto z ;
\end{aligned}
$$

for $C_{13}$, we take

$$
\begin{aligned}
\gamma_{3}: P^{1} \cong C_{34} \text { given by } z \mapsto \frac{1}{1-z}, \\
\gamma_{4}: P^{1} \cong C_{34} \text { given by } z \mapsto \frac{z-1}{z}, \\
\gamma_{34}: P^{1} \cong C_{34} \text { given by } z \mapsto z .
\end{aligned}
$$

15.18. The algebra $R$ (see 4.5 ) is in our case

$$
\mathbf{C}\left[u, u^{-1},(1-u)^{-1}\right] \oplus \mathbf{C}\left[v, v^{-1},(1-v)^{-1}\right] .
$$

(We denote the standard coordinate functions on $C_{12}, C_{34}$ by $u, v$.)

Now $R$ is mapped homomorphically, as in 4.5 (a), to the algebra of formal power series over $\mathbf{C}$ in six different ways by

$$
f \mapsto\left(\begin{array}{ccc}
{ }^{1} f & { }^{2} f & { }^{12} f \\
{ }^{4} f & 3 f & 34 f
\end{array}\right) .
$$

These map $u, v, u(u-1)^{-1}, v(v-1)^{-1} \in R$ as follows:

$$
\begin{array}{rlrl}
u & \mapsto\left(\begin{array}{ccc}
\frac{1}{1-\epsilon} & \frac{\epsilon-1}{\epsilon} & \epsilon \\
0 & 0 & 0
\end{array}\right), & v \mapsto\left(\begin{array}{ccc}
0 & 0 & 0 \\
\frac{\epsilon-1}{\epsilon} & \frac{1}{1-\epsilon} & \epsilon
\end{array}\right), & \\
u(u-1)^{-1} & \mapsto\left(\begin{array}{ccc}
\epsilon^{-1} & 1-\epsilon & \frac{\epsilon}{\epsilon-1} \\
0 & 0 & 0
\end{array}\right), & v(v-1)^{-1} \mapsto\left(\begin{array}{ccc}
0 & 0 & 0 \\
1-\epsilon & \frac{1}{\epsilon} & \frac{\epsilon}{\epsilon-1}
\end{array}\right)
\end{array}
$$

15.19. Let $\Gamma, G_{N}$ be as in $4.6,4.8$, and let $\Gamma_{B_{n}}$ be as in 8.2.

15.20. Let $W=\bigotimes_{i=1}^{4} V_{i}$. For $1 \leq n \leq \infty$, let $W_{n}=B_{n} \otimes W$. We regard $W$ (resp. $W_{n}$ ) as a $\Gamma$-module (resp. $\Gamma_{B_{n}}$-module) as in 8.3 .

As in 8.3, let $\widehat{W}_{n}=\lim _{N} W_{n} / G_{N} W_{n}, \widehat{W}={\underset{\lim }{\longleftarrow}}_{N} W / G_{N} W$. 
Then $\widehat{W}$ (resp. $\widehat{W}_{n}$ ) is naturally a $\widetilde{\mathbf{g}}^{\bigcirc}$-module (resp. $\widetilde{\mathbf{g}}_{B_{n}}^{\odot}$-module) (see 8.4) and the $\widetilde{\mathbf{g}}^{\odot}$-modules $T(W)=\widehat{W}(-\infty)$ and $T(W)^{\sharp}$ (resp. the $\widetilde{\mathbf{g}}_{B_{n}}^{\odot}$-modules $T\left(W_{n}\right)=\widehat{W}_{n}(-\infty)$ and $\left.T\left(W_{n}\right)^{\sharp}\right)$ are well defined.

From 8.12, we have a natural isomorphism of $\widetilde{\mathbf{g}}_{B_{n}}^{\odot}$-modules

$$
B_{n} \otimes T(W)^{\sharp} \cong T\left(W_{n}\right)^{\sharp} .
$$

From 7.12, we have a natural isomorphism of $\widetilde{\mathbf{g}}^{\bigcirc}$-modules

$$
\left(V_{1} \dot{\otimes} V_{2}\right) \otimes\left(V_{3} \dot{\otimes} V_{4}\right) \cong T(W)^{\sharp} .
$$

Tensoring here by $B_{n}$ and composing with the previous isomorphism, we obtain an isomorphism of $\widetilde{\mathbf{g}}_{B_{n}}^{\mathcal{O}}$-modules

$$
B_{n} \otimes\left(V_{1} \dot{\otimes} V_{2}\right) \otimes\left(V_{3} \dot{\otimes} V_{4}\right) \cong T\left(W_{n}\right)^{\sharp} .
$$

Taking coinvariants we obtain an isomorphism of $B_{n}$-modules

$$
\Psi_{n}:\left(B_{n} \otimes\left(V_{1} \dot{\otimes} V_{2}\right) \otimes\left(V_{3} \dot{\otimes} V_{4}\right)\right)_{\Delta 2, n} \cong T\left(W_{n}\right)_{\Delta 2, n}^{\sharp} .
$$

15.21. In our case, the action of $\delta_{12} \epsilon^{m} c+\delta_{34} \epsilon^{m^{\prime}} c^{\prime} \in \widetilde{\mathbf{g}}_{B_{n}}^{\otimes}$ on $\widehat{W}_{n}$ is given by

$$
\begin{aligned}
\left(y_{1}, y_{2}, y_{3}, \ldots\right) \mapsto & \left(\left(u^{m} c\right) y_{k+1},\left(u^{m} c\right) y_{k+2},\left(u^{m} c\right) y_{k+3}, \ldots\right) \\
& +\left(\left(v^{m^{\prime}} c^{\prime}\right) y_{k+1},\left(v^{m^{\prime}} c^{\prime}\right) y_{k+2},\left(v^{m^{\prime}} c^{\prime}\right) y_{k+3}, \ldots\right)
\end{aligned}
$$

for $k \geq 0$ sufficiently large. (See 4.14(a).)

15.22. Now, if $1 \leq n<\infty$, we have $A_{n}=B_{n}$; hence, $W_{n}=Y_{n}$ is both a $\Delta_{4, n}$-module and a $\Gamma_{B_{n}}$-module. This gives sense to the following result.

Lemma 15.23. If $N \geq 2 n-1$, then $G_{N} W_{n} \subset \Delta_{4, n} W_{n}$.

Note that $G_{1}$ is spanned by the elements

$$
\begin{aligned}
& u f(u) c \text { and } v f^{\prime}(v) c^{\prime} \text { with } f(u) \in \mathbf{C}\left[u,(1-u)^{-1}\right], f^{\prime}(v) \in \\
& \mathbf{C}\left[v,(1-v)^{-1}\right], c, c^{\prime} \in \mathbf{g} .
\end{aligned}
$$

Let $f(u) \in \mathbf{C}\left[u,(1-u)^{-1}\right]$ and let $c \in \mathbf{g}$. We define two $B_{n}$-linear maps $T(f, c), T^{\prime}(f, c): W_{n} \rightarrow W_{n}$ by

$$
\begin{aligned}
T(f, c)\left(a x_{1} \otimes x_{2} \otimes x_{3} \otimes x_{4}\right)= & a \frac{1}{1-\epsilon} f\left(\frac{1}{1-\epsilon}\right) c x_{1} \otimes x_{2} \otimes x_{3} \otimes x_{4} \\
& +a x_{1} \otimes \frac{\epsilon-1}{\epsilon} f\left(\frac{\epsilon-1}{\epsilon}\right) c x_{2} \otimes x_{3} \otimes x_{4}, \\
T^{\prime}(f, c)\left(a x_{1} \otimes x_{2} \otimes x_{3} \otimes x_{4}\right)= & a x_{1} \otimes x_{2} \otimes(1-\epsilon) f(t(1-\epsilon)) c x_{3} \otimes x_{4} \\
& +a x_{1} \otimes x_{2} \otimes x_{3} \otimes \frac{\epsilon}{\epsilon-1} f\left(t \frac{\epsilon}{\epsilon-1}\right) c x_{4}
\end{aligned}
$$


for all $x_{i} \in V_{i}$ and $a \in B_{n}$. In the $G_{1}$-module structure of $W_{n}$, we have $u f(u) c=T(f, c)$ as operators on $W_{n}$. In the $\Delta_{4, n}$-module structure of $W_{n}$, we have $p f(p) c=T(f, c)+t T\left(f^{\prime}, c\right)$ as operators on $W_{n}$. (Note that $p f(p) c \in$ $\left.\Delta_{4, n} \cdot\right)$

We now consider $N$ functions $f_{1}, f_{2}, \ldots, f_{N}$ in $\mathbf{C}\left[u,(1-u)^{-1}\right]$ and $N$ elements $c_{1}, c_{2}, \ldots, c_{N}$ of $\mathbf{g}$. We show that

$$
\begin{aligned}
& T\left(f_{1}, c_{1}\right) T\left(f_{2}, c_{2}\right) \cdots T\left(f_{N}, c_{N}\right) W_{n} \\
& \quad \in t^{N} T^{\prime}\left(f_{N}, c_{N}\right) T^{\prime}\left(f_{N-1}, c_{N-1}\right) \cdots T^{\prime}\left(f_{1}, c_{1}\right) W_{n}+\Delta_{4, n} W_{n} .
\end{aligned}
$$

Indeed, the left-hand side is equal to

$$
\left(\left(p f_{1}(p) c_{1}\right)-t T^{\prime}\left(f_{1}, c_{1}\right)\right) T\left(f_{2}, c_{2}\right) \cdots T\left(f_{N}, c_{N}\right) W_{n} .
$$

Now $T^{\prime}\left(f_{1}, c_{1}\right)$ commutes with each of the operators $T\left(f_{k}, c_{k}\right)$ since $T^{\prime}\left(f_{1}, c_{1}\right)$ acts on the last two factors in the tensor product, while $T\left(f_{k}, c_{k}\right)$ acts on the first two factors in the tensor product. Thus, the left-hand side of (b) is contained in

$$
\left(p f_{1}(p) c_{1}\right) T\left(f_{2}, c_{2}\right) \cdots T\left(f_{N}, c_{N}\right) W_{n}+t T\left(f_{2}, c_{2}\right) \cdots T\left(f_{N}, c_{N}\right) T^{\prime}\left(f_{1}, c_{1}\right) W_{n} .
$$

We may assume that (b) holds for $N-1$ factors; we then see that

$$
\begin{aligned}
& T\left(f_{2}, c_{2}\right) \cdots T\left(f_{N}, c_{N}\right) T^{\prime}\left(f_{1}, c_{1}\right) W_{n} \\
& \quad \in t^{N-1} T^{\prime}\left(f_{N}, c_{N}\right) T^{\prime}\left(f_{N-1}, c_{N-1}\right) \cdots T^{\prime}\left(f_{1}, c_{1}\right) W_{n}+\Delta_{4, n} W_{n} .
\end{aligned}
$$

Since $\left(p f_{1}(p) c_{1}\right) T\left(f_{2}, c_{2}\right) \cdots T\left(f_{N}, c_{N}\right) W_{n} \in \Delta_{4, n} W_{n}$, we see that (b) is proved by induction on $N$. (For $N=0$ there is nothing to prove.) If we take $N \geq n$, then $t^{N}=0$ on $W_{n}$; hence, from (b) we have that

$$
\left(u f_{1}(u) c_{1}\right)\left(u f_{2}(u) c_{2}\right) \cdots\left(u f_{N}(u) c_{N}\right) W_{n} \subset \Delta_{4, n} W_{n} .
$$

Similarly, for any $f_{1}^{\prime}, f_{2}^{\prime}, \ldots, f_{M}^{\prime}$ in $\mathbf{C}\left[u,(1-u)^{-1}\right]$ and $c_{1}^{\prime}, c_{2}^{\prime}, \ldots, c_{M}^{\prime}$ of $\mathbf{g}$ we have

$$
\left(v f_{1}^{\prime}(v) c_{1}^{\prime}\right)\left(v f_{2}^{\prime}(v) c_{2}^{\prime}\right) \cdots\left(v f_{M}^{\prime}(v) c_{M}^{\prime}\right) W_{n} \subset \Delta_{4, n} W_{n}
$$

provided that $M \geq n$.

We now consider a product (in $U\left(\Gamma_{B_{n}}\right)$ ) of $2 n-1$ factors of the form $(u f(u) c)$ or $\left(v f^{\prime}(v) c^{\prime}\right)$ where $f, f^{\prime} \in \mathbf{C}\left[u,(1-u)^{-1}\right]$ and $c, c^{\prime} \in \mathbf{g}$. Any factor of the first kind commutes with any factor of the second kind. Hence, our product can be reordered so that the first $n$ factors are all of one kind. But then (c) and (d) show that our product applied to $W_{n}$ is contained in $\Delta_{4, n} W_{n}$. This shows that $G_{2 n-1} W_{n} \subset \Delta_{4, n} W_{n}$. The lemma is proved.

15.24. From the previous lemma we see that the identity map of $W_{n}$ induces a $B_{n}$-linear map $W_{n} / G_{N} W_{n} \rightarrow W_{n} / \Delta_{4, n} W_{n}$ for $N \geq 2 n-1$. These maps induce a $B_{n}$-linear map

$$
\underset{N}{\lim } W_{n} / G_{N} W_{n} \rightarrow W_{n} / \Delta_{4, n} W_{n}
$$


This restricts to a $B_{n}$-linear map $\widehat{W}_{n}(-\infty) \rightarrow W_{n} / \Delta_{4, n} W_{n}$, which may be regarded as a $B_{n}$-linear map

$$
T\left(W_{n}\right)^{\sharp} \rightarrow W_{n} / \Delta_{4, n} W_{n} .
$$

Now $T\left(W_{n}\right)^{\sharp}$ is a $\Delta_{2, n}$-module, via the imbedding $\Delta_{2, n} \subset \widetilde{\mathbf{g}}_{B_{n}}^{O}$.

We show that, for any element $\xi \in \Delta_{2, n}$, the image of $\xi T\left(W_{n}\right)^{\sharp}$ under (b) is zero.

We may assume that $\xi$ is one of the elements $p^{m} c$ or $q^{m} c$ of $\Delta_{2, n}$ where $m \in \mathbf{N}$. (These elements span $\Delta_{2, n}$.) It is enough to show that in the $\widetilde{\mathbf{g}}_{B_{n}}$ module structure of $\widehat{W}_{n}$, the following holds: for any $\left(y_{1}, y_{2}, \ldots\right) \in \widehat{W}_{n}$, the image of

$$
(-1)^{m}\left(\delta_{(12)} \epsilon^{m} c+\delta_{(34)} t^{m} \epsilon^{-m} c\right)\left(y_{1}, y_{2}, \ldots\right)
$$

and of

$$
(-1)^{m}\left(\delta_{(12)} t^{m} \epsilon^{-m} c+\delta_{(34)} \epsilon^{m} c\right)\left(y_{1}, y_{2}, \ldots\right)
$$

under (a) is zero.

By 15.21 , the image of (c) is equal to $(-1)^{m}$ times $\left(u^{m} c\right) y_{k}+\left(t^{m} v^{-m} c\right) y_{k}$ $\bmod \Delta_{4, n} W_{n}$ for sufficiently large $k$; the image of (d) is equal to $(-1)^{m}$ times $\left(t^{m} u^{-m} c\right) y_{k}+\left(v^{m} c\right) y_{k} \bmod \Delta_{4, n} W_{n}$ for sufficiently large $k$. It remains to observe that the $\Delta_{4, n}$-module structure of $W_{n}$ is related to the $\Gamma_{B_{n}}$-module structure on $W_{n}$ by $\left(p^{m} c\right) y=\left(u^{m} c\right) y+\left(t^{m} v^{-m} c\right) y$ and $\left(q^{m} c\right) y=\left(t^{m} u^{-m} c\right) y+$ $\left(v^{m} c\right) y$ for all $y \in W_{n}$. These formulas follow from the definitions (the first of them has been also used in 15.23).

15.25. We now see that the $B_{n}$-linear map $15.24(\mathrm{~b})$ induces by passage to a quotient space, a $B_{n}$-linear map

$$
\Phi_{n}: T\left(W_{n}\right)^{\sharp} / \Delta_{2, n} T\left(W_{n}\right)^{\sharp} \rightarrow W_{n} / \Delta_{4, n} W_{n} .
$$

(Recall that $1 \leq n<\infty$.)

15.26. For any integer $n \geq 1$ we have an obvious surjective $\mathbf{C}$-algebra homomorphism $B_{n+1} \rightarrow B_{n}$. If $M$ is any $B_{n+1}$-module, we have an obvious homomorphism of $B_{n+1}$-modules.

$$
M=B_{n+1} \otimes_{B_{n+1}} M \rightarrow B_{n} \otimes_{B_{n+1}} M
$$

induced by $B_{n+1} \rightarrow B_{n}$.

If $M$ is one of the $B_{n+1}$-modules

$$
\left(B_{n+1} \otimes\left(V_{1} \dot{\otimes} V_{2}\right) \otimes\left(V_{3} \dot{\otimes} V_{4}\right)\right)_{\Delta_{2, n+1}}, T\left(W_{n+1}\right)_{\Delta_{2, n+1}}^{\sharp},\left(W_{n+1}\right)_{\Delta_{4, n+1}}
$$

then, by the right exactness of tensor products, $B_{n} \otimes_{B_{n+1}} M$ is respectively:

$$
\left(B_{n} \otimes\left(V_{1} \dot{\otimes} V_{2}\right) \otimes\left(V_{3} \dot{\otimes} V_{4}\right)\right)_{\Delta_{2, n}}, T\left(W_{n}\right)_{\Delta_{2, n}}^{\sharp}, \quad\left(W_{n}\right)_{\Delta_{4, n}} .
$$


Hence we have a diagram

$$
\begin{array}{ccc}
\left(B_{n+1} \otimes\left(V_{1} \dot{\otimes} V_{2}\right) \otimes\left(V_{3} \dot{\otimes} V_{4}\right)\right)_{\Delta_{2, n+1}} & \longrightarrow & \left(B_{n} \otimes\left(V_{1} \dot{\otimes} V_{2}\right) \otimes\left(V_{3} \dot{\otimes} V_{4}\right)\right)_{\Delta_{2, n}} \\
\Psi_{n+1} \downarrow & \Psi_{n} \downarrow \\
T\left(W_{n+1}\right)_{\Delta_{2, n+1}}^{\sharp} & T & T\left(W_{n}\right)_{\Delta_{2, n}}^{\sharp} \\
\Phi_{n+1} \downarrow & \Phi_{n} \downarrow \\
W_{n+1} / \Delta_{4, n+1} W_{n+1} & \longrightarrow & W_{n} / \Delta_{4, n} W_{n}
\end{array}
$$

with surjective horizontal maps of the type (a) and with $\Psi_{n}, \Psi_{n+1}$ isomorphisms as in $15.20(a)$.

Using the definitions, one checks that the diagram above is commutative.

The following result will be proved in the next section.

Proposition 15.27. The $B_{n}$-linear map $\Phi_{n}$ (see 15.25(a)) is an isomorphism for any integer $n \geq 1$.

\section{Degenneration of Quadrics (II)}

In this section we shall prove Proposition 15.27.

16.1. We fix an integer $n \geq 1$. Let $t, p, q, u, v$ be indeterminates. Let $B_{n}=\mathbf{C}[t] /\left(t^{n}\right)$.

We have homomorphisms of $B_{n}$-algebras

$$
\begin{gathered}
B_{n}[p, q] /(p q-t) \rightarrow B_{n}\left[u, u^{-1}\right] \oplus B_{n}\left[v, v^{-1}\right], \\
B_{n}[[p, q]] /(p q-t) \rightarrow B_{n}((u)) \oplus B_{n}((v)),
\end{gathered}
$$

given by

$$
p \mapsto\left(u, t v^{-1}\right), \quad q \mapsto\left(t u^{-1}, v\right) .
$$

(Note that the map (b) could not be defined without the condition $t^{n}=0$.)

Let $T$ be the subalgebra (without 1) $B_{n}\left[u^{-1}\right] \oplus B_{n}\left[v^{-1}\right] v^{-1}$ of $B_{n}\left[u, u^{-1}\right] \oplus$ $B_{n}\left[v, v^{-1}\right]$.

Lemma 16.2. (a) The homomorphism 16.1(a) is injective; its image is a $B_{n}$ submodule of $B_{n}\left[u, u^{-1}\right] \oplus B_{n}\left[v, v^{-1}\right]$, complementary to $T$.

(b) The homomorphism $16.1(\mathrm{~b})$ is injective; its image is a $B_{n}$-submodule of $B_{n}((u)) \oplus B_{n}((v))$, complementary to $T$.

Let $M$ be the $B_{n}$-submodule of $B_{n}[p, q]$ consisting of all the polynomials

$$
\sum_{k, l \in \mathbf{N}: k l=0} b_{k, l} p^{k} q^{l} \text { with } b_{k, l} \in B_{n} .
$$

The restriction to $M$ of the canonical homomorphism

$$
B_{n}[p, q] \rightarrow B_{n}[p, q] /(p q-t)
$$


is clearly an isomorphism

$$
M \cong B_{n}[p, q] /(p q-t) .
$$

Assume that the image of a polynomial (c) in $M$ under the composition

$$
\begin{aligned}
M & \rightarrow B_{n}[p, q] /(p q-t) \rightarrow B_{n}\left[u, u^{-1}\right] \oplus B_{n}\left[v, v^{-1}\right] \\
& \rightarrow\left(B_{n}\left[u, u^{-1}\right] \oplus B_{n}\left[v, v^{-1}\right]\right) / T
\end{aligned}
$$

is zero. Then

$$
\sum_{k \geq 1} a_{k, 0} u^{k}=0 \text { and } \sum_{l \geq 0} a_{0, l} v^{l}=0
$$

hence, $a_{k, 0}=a_{0, l}=0$ for all $k, l$. Thus the composition (e) is injective. Using (d), it follows that the map 16.1(a) is injective and its image has zero intersection with $T$.

Now any element of the form $\left(u^{k}, 0\right)$ is the sum of an element in the image of 16.1(a) and one in $T$. (When $k \leq 0$ it is in $T$; when $k>0$ it is the sum of the image of $p^{k}$ and of $\left(0,-t^{k} v^{-k}\right) \in T$.)

Similarly, any element of the form $\left(0, v^{l}\right)$ is the sum of an element in the image of 16.1(a) and one in $T$. (When $l<0$ it is in $T$; when $l \geq 0$ it is the sum of the image of $q^{l}$ and of $\left(-t^{k} u^{-l}, 0\right) \in T$.)

This completes the proof of (a). The proof of (b) is entirely similar.

16.3. The previous proof shows also that

$$
B_{n}[p, q] /(p q-t) \text { is a free } B_{n} \text {-module . }
$$

A basis is given by the elements $p^{k}(k>0), q^{l}(l>0)$, and 1 .

16.4. We have a commutative diagram of algebra homomorphisms:

$$
\begin{gathered}
B_{n}[p, q] /(p q-t) \longrightarrow B_{n}\left[u, u^{-1}\right] \oplus B_{n}\left[v, v^{-1}\right] \\
\downarrow \\
B_{n}[[p, q]] /(p q-t) \longrightarrow B_{n}((u)) \oplus B_{n}((v))
\end{gathered}
$$

where the horizontal maps are the imbeddings from the previous lemma and the vertical maps are the obvious ones. Since the right vertical map is clearly an imbedding, the same must hold for the left vertical map. Hence all algebras in the diagram are naturally subalgebras of $B_{n}((u)) \oplus B_{n}((v))$.

Lemma 16.5. We have

$$
\left(B_{n}[[p, q]] /(p q-t)\right) \cap\left(B_{n}\left[u, u^{-1}\right] \oplus B_{n}\left[v, v^{-1}\right]\right)=B_{n}[p, q] /(p q-t)
$$

(intersection inside $B_{n}((u)) \oplus B_{n}((v))$ ).

Let $x$ be an element in the intersection. Since $x \in B_{n}\left[u, u^{-1}\right] \oplus B_{n}\left[v, v^{-1}\right]$, we can write $x=x_{1}+x_{2}$ with $x_{1} \in T$ and $x_{2} \in B_{n}[p, q] /(p q-t)$ (see 16.2(a)).

Since $x \in B_{n}[[p, q]] /(p q-t)$, we have $x-x_{2} \in B_{n}[[p, q]] /(p q-t)$. On the other hand, $x-x_{2} \in T$ and $T$ has zero intersection with $B_{n}[[p, q]] /(p q-t)$. It follows that $x-x_{2}=0$. Thus, $x \in B_{n}[p, q] /(p q-t)$. The lemma is proved. 
16.6. The elements $p-1, q-1$ of $B_{n}[p, q] /(p q-t)$ are not zero divisors since they are invertible in the larger algebra $B_{n}[[p, q]] /(p q-t)$. They generate a multiplicatively closed subset of $B_{n}[p, q] /(p q-t)$; we consider the corresponding ring of fractions

$$
\mathscr{C}=\frac{B_{n}[p, q]}{(p q-t)}\left[(p-1)^{-1},(q-1)^{-1}\right] .
$$

It contains $B_{n}[[p, q]] /(p q-t)$ as a subalgebra.

By exactness of localization, from the commutative diagram of imbeddings in 16.4, we obtain the following commutative diagram of imbeddings:

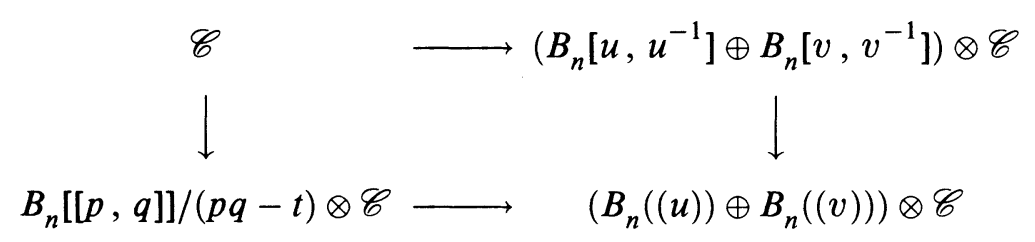

or, equivalently, the following commutative diagram of imbeddings:

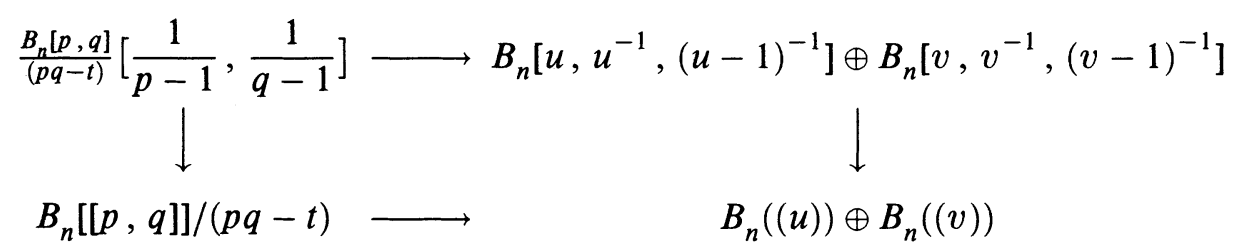

Here we have used the following fact: the subalgebra of $B_{n}((u)) \oplus B_{n}((v))$ generated by $B_{n}\left[u, u^{-1}\right] \oplus B_{n}\left[v, v^{-1}\right]$ and by

$$
\begin{aligned}
& (p-1)^{-1}=\left((u-1)^{-1}, 0\right)+\left(0,\left(t v^{-1}-1\right)^{-1}\right), \\
& (q-1)^{-1}=\left(0,(v-1)^{-1}\right)+\left(\left(t u^{-1}-1\right)^{-1}, 0\right),
\end{aligned}
$$

coincides with $B_{n}\left[u, u^{-1},(u-1)^{-1}\right] \oplus B_{n}\left[v, v^{-1},(v-1)^{-1}\right]$.

This follows immediately from the fact that

$$
\left(t u^{-1}-1\right)^{-1} \in B_{n}\left[u, u^{-1}\right] \text { and }\left(t v^{-1}-1\right)^{-1} \in B_{n}\left[v, v^{-1}\right]
$$

(we use $t^{n}=0$ ).

\section{Lemma 16.7. We have}

$$
\begin{aligned}
& \left(B_{n}[[p, q]] /(p q-t)\right) \cap\left(B_{n}\left[u, u^{-1},(u-1)^{-1}\right] \oplus B_{n}\left[v, v^{-1},(v-1)^{-1}\right]\right) \\
& \quad=\frac{B_{n}[p, q]}{(p q-t)}\left[(p-1)^{-1},(q-1)^{-1}\right] .
\end{aligned}
$$

This follows from the corresponding equality in Lemma 16.5, using exactness of localization.

Lemma 16.8. We have a direct sum decomposition (as $B_{n}$-modules):

$$
\begin{gathered}
B_{n}\left[u, u^{-1},(u-1)^{-1}\right] \oplus B_{n}\left[v, v^{-1},(v-1)^{-1}\right] \\
=\frac{B_{n}[p, q]}{(p q-t)}\left[(p-1)^{-1},(q-1)^{-1}\right] \oplus T
\end{gathered}
$$


We have

$T \subset B_{n}\left[u, u^{-1}\right] \oplus B_{n}\left[v, v^{-1}\right] \subset B_{n}\left[u, u^{-1},(u-1)^{-1}\right] \oplus B_{n}\left[v, v^{-1},(v-1)^{-1}\right]$ and

$\frac{B_{n}[p, q]}{(p q-t)}\left[(p-1)^{-1},(q-1)^{-1}\right] \subset B_{n}\left[u, u^{-1},(u-1)^{-1}\right] \oplus B_{n}\left[v, v^{-1},(v-1)^{-1}\right]$ (see 16.6) and hence

$$
\begin{aligned}
& \frac{B_{n}[p, q]}{(p q-t)}\left[(p-1)^{-1},(q-1)^{-1}\right]+T \\
& \quad \subset B_{n}\left[u, u^{-1},(u-1)^{-1}\right] \oplus B_{n}\left[v, v^{-1},(v-1)^{-1}\right]
\end{aligned}
$$

Now

$$
\frac{B_{n}[p, q]}{(p q-t)}\left[(p-1)^{-1},(q-1)^{-1}\right] \cap T \subset\left(B_{n}[[p, q]] /(p q-t)\right) \cap T=0
$$

(see 16.2(b)).

Let $x \in B_{n}\left[u, u^{-1},(u-1)^{-1}\right] \oplus B_{n}\left[v, v^{-1},(v-1)^{-1}\right]$. By 16.2(b), we can write $x=x_{1}+x_{2}$ with $x_{1} \in T$ and $x_{2} \in B_{n}[[p, q]] /(p q-t)$.

Since

$$
T \subset B_{n}\left[u, u^{-1},(u-1)^{-1}\right] \oplus B_{n}\left[v, v^{-1},(v-1)^{-1}\right],
$$

we have $x-x_{1} \in B_{n}\left[u, u^{-1},(u-1)^{-1}\right] \oplus B_{n}\left[v, v^{-1},(v-1)^{-1}\right]$. Thus, $x_{2}$ belongs to the intersection

$$
\frac{B_{n}[[p, q]]}{(p q-t)} \cap\left(B_{n}\left[u, u^{-1},(u-1)^{-1}\right] \oplus B_{n}\left[v, v^{-1},(v-1)^{-1}\right]\right)
$$

which, by 16.7 , is equal to $\frac{B_{n}[p, q]}{(p q-t)}\left[(p-1)^{-1},(q-1)^{-1}\right]$. Thus, we have $x \in \frac{B_{n}[p, q]}{(p q-t)}\left[(p-1)^{-1},(q-1)^{-1}\right]+T$ and the lemma is proved.

16.9. Let $\varnothing=\{12,34\}$, as in $\S 15$. There is a unique homomorphism of $B_{n}$-Lie algebras

such that

$$
B_{n}[p, q] /(p q-t) \otimes \mathbf{g} \rightarrow \widetilde{\mathbf{g}}_{B_{n}}^{\odot}
$$

$$
p^{m} c \mapsto\left(\epsilon^{m} c, t^{m} \epsilon^{-m} c\right) \text { and } q^{m} c \mapsto\left(t^{m} \epsilon^{-m} c, \epsilon^{m} c\right)
$$

for all $m \geq 0$ and $c \in \mathbf{g}$. This can be checked directly or can be deduced from 15.13(b).

By $16.2(\mathrm{a})$, this homomorphism is injective, so it identifies $\frac{B_{n}[p, q]}{(p q-t)} \otimes \mathbf{g}$ with a Lie subalgebra $P_{0}$ of $\widetilde{\mathbf{g}}_{B_{n}}^{\odot}$. Let $P=P_{0}+B_{n} \mathbf{1}$; this is actually a direct sum and is a $B_{n}$-Lie subalgebra of $\widetilde{\mathbf{g}}_{B_{n}}^{\bigcirc}$.

The formulas (c) extend by continuity to a $B_{n}$-Lie algebra homomorphism

$$
B_{n}[[p, q]] /(p q-t) \otimes \mathbf{g} \rightarrow \widehat{\mathbf{g}}_{B_{n}}^{\odot} .
$$

By 16.2(b), this is injective, so it identifies $B_{n}[[p, q]] /(p q-t) \otimes \mathbf{g}$ with a Lie subalgebra $\widehat{P}_{0}$ of $\widehat{\mathbf{g}}_{B_{n}}^{\mathcal{O}}$. Let $\widehat{P}=\widehat{P}_{0}+B_{n} \mathbf{1}$; this is actually a direct sum and is a $B_{n}$-Lie subalgebra of $\widehat{\mathbf{g}}_{B_{n}}^{\odot}$. 
16.10. The diagonal module. The following $\widetilde{\mathbf{g}}_{B_{n}}^{\odot}$-module has been defined by A. Beilinson (private communication):

$$
X_{n}=U\left(\widetilde{\mathbf{g}}_{B_{n}}^{\odot}\right) \otimes_{U(P)} B_{n}
$$

here, $B_{n}$ is regarded as a $P$-module in which $P_{0}$ acts as zero and 1 acts as multiplication by $\kappa-h$.

We call $X_{n}$ the diagonal module.

16.11. Let $Q$ be the Lie subalgebra $\left(B_{n}\left[u^{-1}\right] \oplus B_{n}\left[v^{-1}\right] v^{-1}\right) \otimes \mathbf{g}$ of $\widetilde{\mathbf{g}}_{B_{n}}^{\odot}$. From 16.2(a), we see that we have a direct sum decomposition $\widetilde{\mathbf{g}}_{B_{n}}^{\odot}=P \oplus Q$ (as $B_{n}$-modules).

Using A.2(a) (which is applicable, since the Lie algebras involved are free over $B_{n}$; see 16.3$)$ we see that $\left(\widetilde{\mathbf{g}}_{B_{n}}^{\odot}, Q, P, B_{n}\right)$ is a split induction datum and that $X_{n}$ may be identified with $U(Q)$ with the unique $\tilde{\mathbf{g}}_{B_{n}}^{\odot}$-module structure such that $Q$ acts by left multiplication and

$$
P_{0} \cdot 1=0, \quad(b \mathbf{1}) 1=(\kappa-h) b 1 \quad \text { for all } b \in B_{n} .
$$

(Here 1 is the unit element of $U(Q)$.)

By the lemma below, this $\widetilde{\mathbf{g}}_{B_{n}}^{\mathcal{O}}$-module is smooth. Hence it extends naturally to a $\widehat{\mathbf{g}}_{B_{n}}^{\mathcal{O}}$-module. Hence there is a (unique) $\widehat{\mathbf{g}}_{B_{n}}^{\mathcal{O}}$-module structure on $U(Q)$ such that $Q$ acts by left multiplication and

$$
\widehat{P}_{0} \cdot 1=0, \quad(b \mathbf{1}) 1=(\kappa-h) b 1 \text { for all } b \in B_{n} .
$$

This shows that

$$
\left(\widehat{\mathbf{g}}_{B_{n}}^{\odot}, Q, \widehat{P}, B_{n}\right) \text { is a split induction datum. }
$$

By A.2(b), we therefore have

$$
X_{n}=U\left(\widehat{\mathbf{g}}_{B_{n}}^{\odot}\right) \otimes_{U(\widehat{P})} B_{n}
$$

We have the following result.

Lemma 16.12. We have $X_{n}=X_{n}(\infty)$.

For any $c_{1}, \ldots, c_{r} \in \mathbf{g}$ we have

$$
\left(u c_{1}\right)\left(u c_{2}\right) \cdots\left(u c_{r}\right)(1)=(-1)^{r} t^{r}\left(v^{-1} c_{r}\right) \cdots\left(v^{-1} c_{2}\right)\left(v^{-1} c_{1}\right)(1) .
$$

This follows immediately by induction on $r$ using the identity

$$
(u c)(1)=-t\left(v^{-1} c\right)(1)
$$

which holds for any $c \in \mathbf{g}$ since $0=(p c)(1)=(u c)(1)+\left(t v^{-1} c\right)(1)$. Similarly we have

$$
\left(v c_{1}^{\prime}\right)\left(v c_{2}^{\prime}\right) \cdots\left(v c_{s}^{\prime}\right)(1)=(-1)^{s} t^{s}\left(u^{-1} c_{s}^{\prime}\right) \cdots\left(u^{-1} c_{2}^{\prime}\right)\left(u^{-1} c_{1}^{\prime}\right)(1)
$$


for any $c_{1}^{\prime}, \ldots, c_{s}^{\prime} \in \mathbf{g}$. Since $t^{n}=0$, it follows that

$$
\begin{aligned}
& \left(u c_{1}\right)\left(u c_{2}\right) \cdots\left(u c_{r}\right)\left(v c_{1}^{\prime}\right)\left(v c_{2}^{\prime}\right) \cdots\left(v c_{s}^{\prime}\right)(1) \\
& =\left(v c_{1}^{\prime}\right)\left(v c_{2}^{\prime}\right) \cdots\left(v c_{s}^{\prime}\right)\left(u c_{1}\right)\left(u c_{2}\right) \cdots\left(u c_{r}\right)(1)
\end{aligned}
$$

is zero if at least one of the inequalities $r \geq n, s \geq n$ holds. This condition is certainly satisfied if $r+s \geq 2 n-1$.

Thus, we have $1 \in X_{n}(2 n-1)$; hence, $1 \in X_{n}(\infty)$. Now 1 generates $X_{n}$ as a $\widetilde{\mathbf{g}}_{B_{n}}^{\odot}$-module; on the other hand, $X_{n}(\infty)$ is a $\widetilde{\mathbf{g}}_{B_{n}}^{\odot}$-submodule of $X_{n}$. It follows that $X_{n}(\infty)=X_{n}$.

16.13. In the setup of $\S 15$, we consider the $B_{n}$-Lie algebra $\mathbf{h}=\widehat{\mathbf{g}}_{B_{n}}^{\odot}$ and its Lie subalgebras $\mathbf{h}^{+}=\widehat{P}, \mathbf{h}^{-}=Q$.

Let

$$
\begin{aligned}
\mathbf{h}^{\prime} & =\left(B_{n}\left[u, u^{-1},(u-1)^{-1}\right] \oplus B_{n}\left[v, v^{-1},(v-1)^{-1}\right]\right) \otimes \mathbf{g} \oplus B_{n} \mathbf{1}=\Gamma_{B_{n}}, \\
\mathbf{h}^{\prime+} & =\frac{B_{n}[p, q]}{(p q-t)}\left[(p-1)^{-1},(q-1)^{-1}\right] \otimes \mathbf{g} \oplus B_{n} \mathbf{1} .
\end{aligned}
$$

We have natural imbeddings of $B_{n}$-modules $\mathbf{h}^{\prime+} \subset \mathbf{h}^{\prime} \subset \mathbf{h}$ induced by the maps in the diagram in 16.6 and taking $\mathbf{1}$ to $\mathbf{1}$. In this way, $\mathbf{h}^{\prime+}$ and $\mathbf{h}^{\prime}$ become Lie subalgebras of $\mathbf{h}$.

Let $\mathbf{h}^{\prime-}=Q$. We have $\mathbf{h}=\mathbf{h}^{-} \oplus \mathbf{h}^{+}, \mathbf{h}^{\prime}=\mathbf{h}^{\prime-} \oplus \mathbf{h}^{+}$as $B_{n}$-modules. (See 16.2(b), 16.8(a).)

Hence we may apply Proposition A.5 to these Lie algebras, to the $\mathbf{h}^{+}$-module $B_{n}$ (as in 16.10), and, to the $\mathbf{h}^{\prime}$-module $W_{n}=B_{n} \otimes\left(\otimes_{i=1}^{4} V_{i}\right)$. Note that the hypothesis of A.5 that $\left(\mathbf{h}, \mathbf{h}^{-}, \mathbf{h}^{+}, V\right)$ is a split induction datum is satisfied by 16.11(a). From A.5, we see that the $B_{n}$-linear map

$$
\left(W_{n}\right)_{\mathbf{h}^{\prime+}} \rightarrow\left(W_{n} \otimes_{B_{n}} X_{n}\right)_{\Gamma_{B_{n}}}
$$

induced on coinvariants by the imbedding $W_{n} \rightarrow W_{n} \otimes_{B_{n}} X_{n}$ given by $w \mapsto$ $w \otimes 1$, is an isomorphism.

Next we consider the diagram

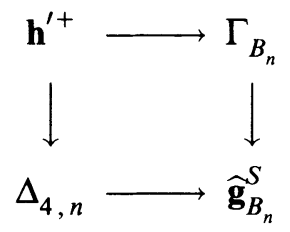

where the upper horizontal map is the imbedding $\mathbf{h}^{\prime+} \subset \mathbf{h}^{\prime}$ considered above, the lower horizontal map is defined by the formulas in 15.9, the left vertical map is the obvious surjection taking 1 to 0 , and the right vertical map is given by the formulas in 15.18 .

It is easy to check that this diagram is commutative. Hence, to take coinvariants for the restriction of the $\widehat{\mathbf{g}}_{B_{n}}^{S}$-module $W_{n}$ to $\mathbf{h}^{\prime}$ via the north-east route (on which $\mathbf{1}$ acts as zero) is the same as to take coinvariants for the restriction 
of $W_{n}$ to $\Delta_{4, n}$ as we did in $\S 15$. Hence from the isomorphism (a) we obtain the following result.

Lemma 16.14. The $B_{n}$-linear map

$$
\left(W_{n}\right)_{\Delta_{4, n}} \rightarrow\left(W_{n} \otimes_{B_{n}} X_{n}\right)_{\Gamma_{B_{n}}}
$$

induced on coinvariants by the imbedding $W_{n} \rightarrow W_{n} \otimes_{B_{n}} X_{n}$ given by $w \mapsto w \otimes 1$ is an isomorphism.

16.15. In the setup of $\S 15$, we consider the $B_{n}$-Lie algebra $\mathbf{h}=\widehat{\mathbf{g}}_{B_{n}}^{\odot}$ and its Lie subalgebras $\mathbf{h}^{+}=\widehat{P}, \mathbf{h}^{-}=Q$.

Let

$$
\begin{aligned}
\mathbf{h}^{\prime} & =\left(B_{n}\left[u, u^{-1}\right] \oplus B_{n}\left[v, v^{-1}\right]\right) \otimes \mathbf{g} \oplus B_{n} \mathbf{1}=\widetilde{\mathbf{g}}_{B_{n}}^{\odot}, \\
\mathbf{h}^{\prime+} & =B_{n}[p, q] /(p q-t) \otimes \mathbf{g} \oplus B_{n} \mathbf{1}
\end{aligned}
$$

We have natural imbeddings of $B_{n}$-modules $\mathbf{h}^{\prime+} \subset \mathbf{h}^{\prime} \subset \mathbf{h}$ induced by the maps in the diagram in 16.4 and taking $\mathbf{1}$ to $\mathbf{1}$. In this way, $\mathbf{h}^{\prime+}$ and $\mathbf{h}^{\prime}$ become sub-Lie algebras of $\mathbf{h}$.

Let $\mathbf{h}^{\prime-}=Q$. We have $\mathbf{h}=\mathbf{h}^{-} \oplus \mathbf{h}^{+}, \mathbf{h}^{\prime}=\mathbf{h}^{\prime-} \oplus \mathbf{h}^{\prime+}$ as $A$-modules. (See 16.2.)

Hence we may apply Proposition A.5 to these Lie algebras, to the $\mathbf{h}^{+}$-module $B_{n}$ (as in 16.10), and to the $\mathbf{h}^{\prime}$-module $T\left(W_{n}\right)$. Note that the hypothesis of A.5 that $\left(\mathbf{h}, \mathbf{h}^{-}, \mathbf{h}^{+}, V\right)$ is a split induction datum is satisfied.

From A.5, we see that the $B_{n}$-linear map

$$
T\left(W_{n}\right)_{\mathbf{h}^{\prime+}} \rightarrow\left(T\left(W_{n}\right) \otimes_{B_{n}} X_{n}\right)_{\tilde{\mathbf{g}}_{B_{n}}^{\odot}}
$$

induced on coinvariants by the imbedding $T\left(W_{n}\right) \rightarrow T\left(W_{n}\right) \otimes_{B_{n}} X_{n}$ given by $w \mapsto w \otimes 1$ is an isomorphism.

Next, we consider the diagram

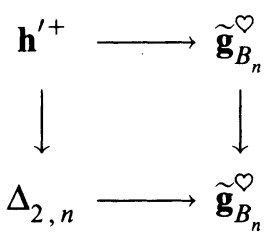

where the upper horizontal map is the imbedding $\mathbf{h}^{\prime+} \subset \mathbf{h}^{\prime}$ considered above, the lower horizontal map is defined by the formulas in 15.13 , the left vertical map is the obvious surjection taking 1 to 0 , and the right vertical map is given by $\sharp$.

A direct check shows that this diagram is commutative. It follows that we may replace in (a) $T\left(W_{n}\right)_{\mathbf{h}^{\prime}}$ by $T\left(W_{n}\right)_{\Delta_{2, n}}^{\sharp}$ (as in $\left.\S 15\right)$ and obtain the following result. 
Lemma 16.16. The $B_{n}$-linear map

$$
T\left(W_{n}\right)_{\Delta_{2, n}}^{\sharp} \rightarrow\left(T\left(W_{n}\right) \otimes_{B_{n}} X_{n}\right)_{\widetilde{\mathbf{g}}_{B_{n}}^{\odot}}
$$

induced on coinvariants by the imbedding $T\left(W_{n}\right) \rightarrow T\left(W_{n}\right) \otimes_{B_{n}} X_{n}$ given by $w \mapsto w \otimes 1$ is an isomorphism.

16.17. Proof of Proposition 15.27. Consider the $B_{n}$-linear map

$$
\left(T\left(W_{n}\right) \otimes_{B_{n}} X_{n}\right)_{\widetilde{\mathbf{B}}_{B_{n}}^{\odot}} \rightarrow\left(W_{n} \otimes_{B_{n}} X_{n}\right)_{\Gamma}
$$

defined as in 8.5.

By 16.16, the left-hand side of (a) is isomorphic to $T\left(W_{n}\right)_{\Delta_{2, n}}^{\sharp}$ (of $\S 15$ ), which is finitely generated by $15.16(\mathrm{a})$ and $15.20(\mathrm{a})$.

By 16.14, the right-hand side of (a) is isomorphic to $\left(W_{n}\right)_{\Delta_{4, n}}$ (of $\left.\S 15\right)$, which is finitely generated by 15.12 .

Since any finitely generated $B_{n}$-module is reflexive, both sides of (a) are reflexive $B_{n}$-modules. Therefore, 8.14 is applicable and shows that (a) is an isomorphism. (Recall that $X_{n}$ is smooth.)

Composing the isomorphism (a) with the isomorphisms 16.16, 16.14, we obtain an isomorphism $\left(T\left(W_{n}\right)\right)_{\Delta_{2, n}} \cong\left(W_{n}\right)_{\Delta_{4, n}}$. It is easy to verify that this coincides with the map $\Phi_{n}$ given in $15.25(\mathrm{a})$.

Hence, that map is an isomorphism and the proposition is proved.

\section{Degeneration OF QUADRICS AND CONNECTIONS}

The main result of this section is Theorem 17.29, which is a form of the associativity isomorphism at the level of coinvariants.

17.1. We preserve the set-up of $\S 15$.

Let $\dot{C}=\{t \in \mathbf{C} \mid t \neq 0,1\}$ and $\dot{C}^{\prime}=\left\{(t, p, q) \in \mathbf{C}^{3} \mid p q=t ; t \neq 0,1 ; p \neq\right.$ $1 ; q \neq 1\}$.

Let $t \in \dot{C}$. Let $\gamma_{i}: P^{1} \cong K_{t}$ be the isomorphism defined in 15.3. If $i \neq j$, then $\gamma_{j}^{-1} \gamma_{i}$ is an automorphism of $P^{1}$ and, hence, is an element of $P G L_{2}(\mathbf{C})$. For any $i_{0} \in S$, we may consider the element of $P G L_{2}(\mathbf{C})^{4}$ whose $i$ th component is $\gamma_{i_{0}}^{-1} \gamma_{i}$. The image of this quadruple in $P G L_{2}(\mathbf{C}) \backslash P G L_{2}(\mathbf{C})^{4}$ is clearly independent of the choice of $i_{0}$. Thus we obtain a map $\dot{C}_{0} \rightarrow$ $P G L_{2}(\mathbf{C}) \backslash P G L_{2}(\mathbf{C})^{4}$; its image is clearly contained in the open subset $\mathscr{V}$ (see 9.2) so that we have obtained a morphism $\nu: \dot{C} \rightarrow \mathscr{V}$.

We can write it explicitly as follows (we choose $i_{0}=2$ in the previous definition; as we have seen, this choice is irrelevant):

$$
\nu(t)=\left(\begin{array}{cc}
\left(\begin{array}{cc}
1 & -1 \\
1 & 0
\end{array}\right) & \left(\begin{array}{cc}
1 & 0 \\
0 & 1
\end{array}\right) \\
\left(\begin{array}{cc}
-1 & 1 \\
t-1 & 1
\end{array}\right) & \left(\begin{array}{cc}
0 & -1 \\
-t & t-1
\end{array}\right)
\end{array}\right)
$$


This can be inserted in a cartesian diagram

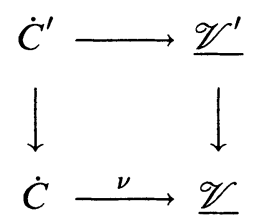

whose right side is as in 9.2. The left vertical map is $(t, p, q) \mapsto t$.

The lower horizontal map is $\nu$. The top horizontal map is $(t, p, q) \mapsto\left(\nu(t),(1-p)^{-1}\right)$. In order for this map to be well defined, we should check that the four matrices in (a) take 0 to four distinct points on $P^{1}$ which are also distinct from $(1-p)^{-1}$. (In our case these four points are $\left.\left(\begin{array}{cc}\infty & 0 \\ 1 & (1-t)^{-1}\end{array}\right).\right)$

\subsection{Let}

$$
\dot{A}=\mathbf{C}[\dot{C}], \quad \dot{A}^{\prime}=\mathbf{C}\left[\dot{C}^{\prime}\right]=\dot{A} \otimes_{A_{\infty}} A_{\infty}^{\prime} .
$$

The commutative diagram in $\mathbf{1 7 . 1}$ gives a commutative diagram of algebra homomorphisms

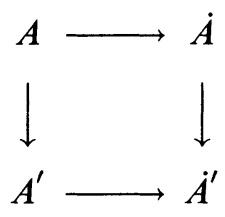

(recall that $A^{\prime}=\mathbf{C}\left[\mathscr{\mathscr { V }}^{\prime}\right]$ and $A=\mathbf{C}[\underline{\mathscr{V}}]$ ).

Using the definitions, we see that we have the following commutative diagram

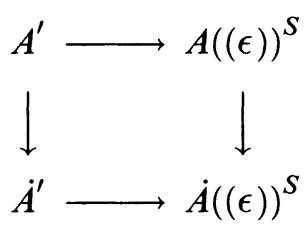

where the vertical maps are induced by the morphisms in (a), the upper horizontal map is defined in 9.4, and the lower horizontal map is induced by the upper one.

Under the natural algebra homomorphisms $A_{\infty}^{\prime} \subset \dot{A}$ and $A^{\prime} \rightarrow \dot{A}^{\prime}$, the function $f_{i} \in A_{\infty}^{\prime}$ (see 15.6) and the function $f_{i} \in A^{\prime}$ (see 9.5) is mapped to the same function in $\dot{A}^{\prime}$, denoted again $f_{i}$.

Lemma 17.3. The functions

$$
f_{i}^{k} \quad(i \in S ; k \geq 1) \text { and } 1
$$

form a basis of $A^{\prime}$ as an $A$-module, of $A_{\infty}^{\prime}$ as an $A_{\infty}$-module, and of $\dot{A}^{\prime}$ as an A-module.

For $A^{\prime}$ and $A_{\infty}^{\prime}$, this has been proved in 9.6, 15.7. The proof for $\dot{A}^{\prime}$ is identical. 
17.4. From the previous lemma we deduce that

$$
\dot{A}^{\prime}=\dot{A} \otimes_{A} A^{\prime}=\dot{A} \otimes_{A_{\infty}} A_{\infty}^{\prime}
$$

17.5. Consider the Lie algebra $\dot{\Delta}_{4}=\dot{A}^{\prime} \otimes \mathbf{g}$.

The $A_{\infty}$-Lie algebra homomorphism $\Delta_{4, \infty} \rightarrow \widehat{\mathbf{g}}_{A_{\infty}}^{S}$ (see 15.9) induces an $\dot{A}$-Lie algebra homomorphism

$$
\dot{\Delta}_{4} \rightarrow \widehat{\mathbf{g}}_{\dot{A}}^{S}
$$

Let $\dot{Y}=\dot{A} \otimes_{A_{\infty}} Y_{\infty}$. By 1.13, $\dot{Y}$ is naturally a $\widehat{\mathbf{g}}_{\dot{A}}^{S}$-module and hence, via (a), a $\dot{\Delta}_{4}$-module.

By the right exactness of tensor products, we have

$$
\dot{Y} / \dot{\Delta}_{4} \dot{Y}=\dot{A} \otimes_{A_{\infty}}\left(Y_{\infty} / \Delta_{4, \infty} Y_{\infty}\right) \text {. }
$$

17.6. Recall that $Y=A \otimes\left(\otimes_{i} V_{i}\right)$ is a $\Delta$-module (see 9.11). We have $\dot{Y}=$ $\dot{A} \otimes_{A} Y$. From 17.4 we see that $\dot{\Delta}_{4}=\dot{A} \otimes_{A} \Delta$, and the $\dot{\Delta}_{4}$-module structure on $\dot{Y}$ is obtained from the $\Delta$-module structure on $Y$ by extension of scalars. Using again the right exactness of tensor products, we see that

$$
\dot{Y} / \dot{\Delta}_{4} \dot{Y}=\dot{A} \otimes_{A}(Y / \Delta Y) \text {. }
$$

From this and 12.12, we deduce that

$$
\dot{Y} / \dot{\Delta}_{4} \dot{Y} \text { is a finitely generated, projective } \dot{A} \text {-module. }
$$

17.7. Since the $A$-module $Y / \Delta Y$ has a natural connection (see 12.11 ), there is, by a general construction, an induced connection on the $\dot{A}$-module $\dot{A} \otimes_{A}$ $(Y / \Delta Y)$.

We shall make explicit this connection in our case. It suffices to describe the operator $\nabla_{\partial / \partial t}$ in this induced connection, for the vector field $\frac{\partial}{\partial t}$ on $\dot{C}$. (This vector field gives a basis of the tangent space of $\dot{C}$ at each point.)

Note that $\dot{C}$ is a smooth, closed subvariety of $\mathscr{V}$, via $\nu$ (see $17.1(\mathrm{a})$ ).

We regard $\frac{\partial}{\partial t}$ as a section of the tangent bundle of $\mathscr{V}$ defined on the submanifold $\nu(\dot{C})$. We extend it to a global section of this tangent bundle; this is a vector field $D$ on $\mathscr{V}$.

Let $y \in Y / \Delta Y$ and let $a \in \dot{A}$. By definition,

$$
\nabla_{\partial / \partial t}(a y)=(\partial a / \partial t) y+\left.a D(y)\right|_{\dot{C}}
$$

This is well defined (independent of the choice of $D$ ) and defines a connection on the $\dot{A}$-module $\dot{A} \otimes_{A}(Y / \Delta Y)$.

17.8. In our case, we can take $D$ to be defined by the following vector field on $G L_{2}(\mathbf{C})^{S}$ :

$$
-\frac{\partial}{\partial_{g_{21 ; 3}}}+\frac{\partial}{\partial_{g_{22 ; 3}}}+\frac{\partial}{\partial_{g_{21 ; 4}}}
$$


where $g_{a b ; i}$ is the $a b$-matrix entry on the $i$ th copy of $G L_{2}(\mathbf{C})$. Recall from 10.2 that

$$
\frac{\partial}{\partial_{g_{21}}}=d^{-1}\left(-g_{11} \theta_{1}-g_{12} \theta_{0}\right), \quad \frac{\partial}{\partial_{g_{22}}}=d^{-1}\left(-g_{11} \theta_{0}-g_{12} \theta_{-1}\right),
$$

where $d$ is the determinant. It follows that $D$ has the same restriction to $\nu(\dot{C})$ as the vector field

$$
t^{-1}\left(\theta_{0,3}-\theta_{-1,3}+\theta_{0,4}-\theta_{1,4}\right) .
$$

Let us now define a C-linear map $\mathscr{H}: \bigotimes_{i} V_{i} \rightarrow \bigotimes_{i} V_{i}$ by

$$
\begin{aligned}
\mathscr{H}\left(y_{1} \otimes y_{2} \otimes y_{3} \otimes y_{4}\right)= & y_{1} \otimes y_{2} \otimes\left(L_{0}-L_{-1}\right)\left(y_{3}\right) \otimes y_{4} \\
& +y_{1} \otimes y_{2} \otimes y_{3} \otimes\left(L_{0}-L_{1}\right)\left(y_{4}\right)
\end{aligned}
$$

for all $y_{i} \in V_{i}$.

We deduce the following result:

Lemma 17.9. The induced connection $\nabla$ on $\dot{Y} / \dot{\Delta}_{4} \dot{Y}$ can be characterized as follows. The operator $\nabla_{t \partial / \partial t}: \dot{Y} / \dot{\Delta}_{4} \dot{Y} \rightarrow \dot{Y} / \dot{\Delta}_{4} \dot{Y}$ makes the diagram

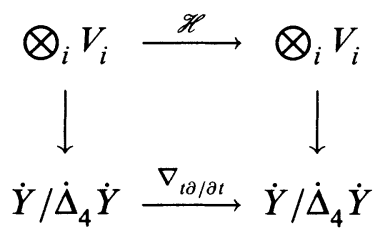

commutative. (Here the vertical maps are given by the composition of the obvious maps $\bigotimes_{i} V_{i} \rightarrow \dot{A} \otimes\left(\bigotimes_{i} V_{i}\right) \rightarrow \dot{Y} / \dot{\Delta}_{4} \dot{Y}$. Note that the image of a vertical map generates $\dot{Y} / \dot{\Delta}_{4} \dot{Y}$ as an $\dot{A}$-module.)

17.10. Let $\mathscr{M}=Y_{\infty} / \Delta_{4, \infty} Y_{\infty}$ and let $\dot{\mathscr{M}}=\dot{Y} / \Delta_{4} \dot{Y}$.

We know that $\mathscr{M}$ is a finitely generated module over the principal ideal domain $A_{\infty}$. Let $\tau(\mathscr{M})$ be its torsion module. Then $\tau(\mathscr{M})$ is finite dimensional over $\mathrm{C}$ and $\mathscr{M} / \tau(\mathscr{M})$ is free of finite rank over $A_{\infty}$. Now $\dot{\mathscr{M}}=\dot{A} \otimes_{A_{\infty}} \mathscr{M}$ is a projective $\dot{A}$-module. It follows that $\tau(\mathscr{M})$ is supported at 0 (i.e., it is annihilated by a power of $t$ ) and that the natural map

$$
\mathscr{M} \rightarrow \dot{A} \otimes_{A_{\infty}} \mathscr{M}=\dot{\mathscr{M}}
$$

(induced by the obvious imbedding $A_{\infty} \subset \dot{A}$ ) has kernel precisely equal to $\tau(\mathscr{M})$.

Note that the C-linear map $\nabla_{t \partial / \partial t}: \dot{\mathscr{M}} \rightarrow \dot{\mathscr{M}}$ (see 17.9) leaves stable the image of the map (a).

This follows from the fact that the commutative diagram in 17.9 can be 
naturally extended to a commutative diagram

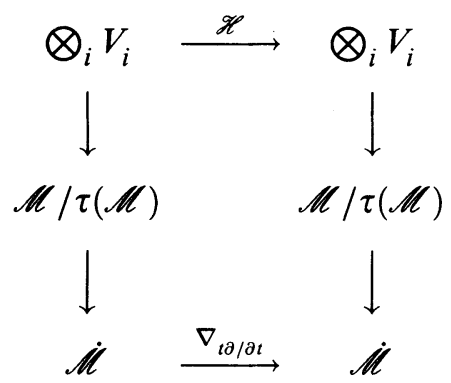

in which $\bigotimes_{i} V_{i} \rightarrow \mathscr{M} / \tau(\mathscr{M})$ is the obvious map (whose image generates $\mathscr{M} / \tau(\mathscr{M})$ as an $A_{\infty}$-module).

Hence, there is a unique C-linear map $\tilde{\nabla}_{t \partial / \partial t}: \mathscr{M} \rightarrow \mathscr{M} / \tau(\mathscr{M})$ which is zero on $\tau(\mathscr{M})$ and is such that the following diagram is commutative.

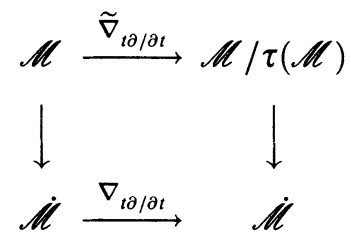

17.11. We can find exact sequences in $\mathscr{O}_{\kappa}: 0 \rightarrow V_{i}^{\prime \prime} \rightarrow V_{i}^{\prime} \rightarrow V_{i} \rightarrow 0$ (for each $i$ ) such that each $V_{i}^{\prime}$ is a generalized Weyl module. Let $Y_{\infty}^{\prime \prime}=A_{\infty} \otimes$ $\left(\otimes_{i} V_{i}^{\prime \prime}\right)$ and $y_{\infty}^{\prime}=A_{\infty} \otimes\left(\otimes_{i} V_{i}^{\prime}\right)$. We form $\mathscr{M}^{\prime \prime}=Y_{\infty}^{\prime \prime} / \Delta_{4, \infty} Y_{\infty}^{\prime \prime}$ and $\mathscr{M}^{\prime}=$ $Y_{\infty}^{\prime} / \Delta_{4, \infty} Y_{\infty}^{\prime}$. By the right exactness of tensor products, we have a natural exact sequence

$$
\mathscr{M}^{\prime \prime} \rightarrow \mathscr{M}^{\prime} \rightarrow \mathscr{M} \longrightarrow 0
$$

By 9.15 , the $A_{\infty}$-module $\mathscr{M}^{\prime}$ is torsion free. Hence we have a commutative diagram with exact rows

(a)

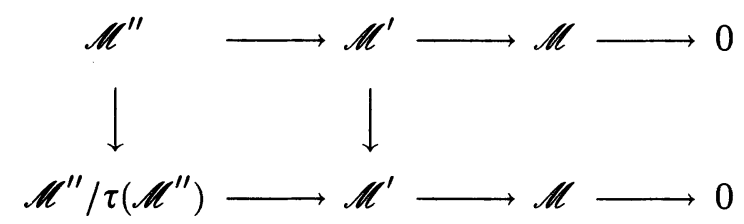

where the vertical maps are of the form $\widetilde{\nabla}_{t \partial / \partial t}\left(\right.$ for $\left.\mathscr{M}^{\prime \prime}, \mathscr{M}^{\prime}\right)$.

From this we see that there is a unique C-linear map $\nabla_{t \partial / \partial t}: \mathscr{M} \rightarrow \mathscr{M}$ which, when added to the diagram as the third vertical map, leaves the diagram commutative.

We consider the diagrams

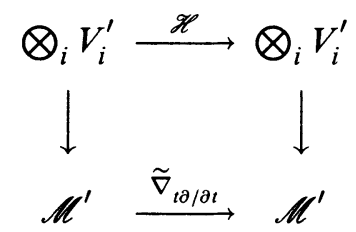




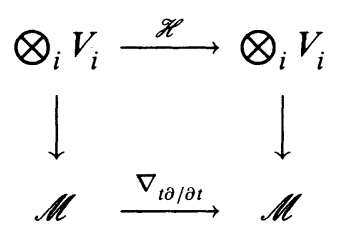

with obvious vertical maps. The first diagram is commutative, by definition. We want to prove that the second one is also commutative. For this purpose, we will complete the two diagrams above to a cubic diagram in which our two diagrams appear as two opposite faces of the cube; namely, we have a natural map from each module in the first diagram to the module in the same position in the second diagram. In the resulting cube, five out of the six bounding squares form commutative diagrams. Moreover, the arrows $\bigotimes_{i} V_{i}^{\prime} \rightarrow \bigotimes_{i} V_{i}$ are surjective. This implies automatically that the sixth bounding square of the cube is a commutative diagram. Thus, the second diagram above is commutative.

17.12. The map $\nabla_{t \partial / \partial t}: \mathscr{M} \rightarrow \mathscr{M}$ satisfies the identity

$$
\nabla_{t \partial / \partial t}(a m)=a \nabla_{t \partial / \partial t}(m)+t(\partial a / \partial t) m
$$

for all $a \in A_{\infty}$ and all $m \in \mathscr{M}$.

Indeed, this follows from the analogous identity for $\tilde{\nabla}_{t \partial / \partial t}: \mathscr{M}^{\prime} \rightarrow \mathscr{M}^{\prime}$, which in turn follows from the imbedding $\mathscr{M}^{\prime} \subset \dot{\mathscr{M}}^{\prime}$ and the fact that $\nabla_{t \partial / \partial t}$ is a connection operator on $\dot{\mathscr{M}}^{\prime}$.

Thus we have the following result.

There is a unique C-linear map $\nabla_{t \partial / \partial t}: \mathscr{M} \rightarrow \mathscr{M}$, such that (a) is satisfied and such that the diagram 17.11(c) is commutative.

(The existence has been proved. The uniqueness is immediate.)

This map can be also characterized by the fact that it is functorial in the $V_{i}$ and that it makes the diagram

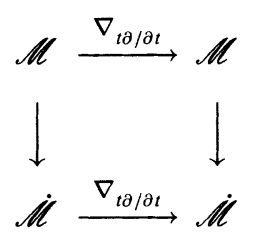

commutative.

17.13. Let $\mathscr{R}$ be the coordinate ring of a Zariski open subset of $\mathbf{C}$ containing 0 . Let $M$ be a finitely generated $\mathscr{R}$-module. We define a connection with a regular singularity at 0 on $M$ to be a C-linear map $\nabla_{t \partial / \partial t}: M \rightarrow M$ such that

$$
\nabla_{t \partial / \partial t}(a m)=a \nabla_{t \partial / \partial t}(m)+t(\partial a / \partial t) m
$$

for all $a \in \mathscr{R}$ and all $m \in M$.

This concept is more general than that of a connection in the usual sense, since the operator $\nabla_{\partial / \partial t}$ is not defined on $M$. 
If we are given an integer $n \geq 1$ and a connection with a regular singularity at 0 on $M$, we have (using (a)):

$$
\nabla_{t \partial / \partial t}\left(t^{n} M\right) \subset t^{n} M
$$

hence, $\nabla_{t \partial / \partial t}$ induces a C-linear endomorphism of $M / t^{n} M$, denoted again $\nabla_{t \partial / \partial t}$. This endomorphism of $M / t^{n} M$ satisfies again (a) for $a \in \mathscr{R} /\left(t^{n}\right)=$ $\mathrm{C}[t] /\left(t^{n}\right)$ and $m \in M / t^{n} M$. Note that $t(\partial a / \partial t)$ is a well-defined derivation of the C-algebra $\mathbf{C}[t] /\left(t^{n}\right)$ (in fact, it is a generator of the $\mathbf{C}[t] /\left(t^{n}\right)$-module of derivations of this algebra).

To give a connection on a $\mathbf{C}[t] /\left(t^{n}\right)$-module is equivalent to giving a $\mathbf{C}$-linear endomorphism $\nabla_{t \partial / \partial t}$ of that module whose commutator with multiplication by $t$ is equal to multiplication by $t$.

Hence, $\nabla_{t \partial / \partial t}$ defines a connection on the $\mathbf{C}[t] /\left(t^{n}\right)$-module $M / t^{n} M$.

17.14. We can summarize our results above as follows: the $A_{\infty}$-module $\mathscr{M}$ has a natural connection with a regular singularity at 0 .

For any integer $n \geq 1$, this induces a connection (in the usual sense) on the $\mathrm{C}[t] /\left(t^{n}\right)$-module

$$
\mathscr{M}_{n}=A_{n} \otimes_{A_{\infty}} \mathscr{M}=\mathscr{M} / t^{n} \mathscr{M}=Y_{n} / \Delta_{4, n} Y_{n} .
$$

This connection makes the following diagram commutative:

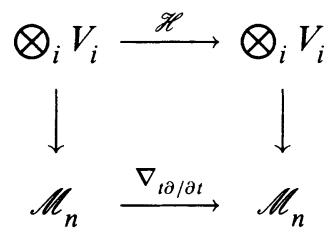

where the vertical maps are the obvious ones.

17.15. We shall now consider a simpler version of the results above (in which 4 is replaced by 2). We place ourselves in the setup of 15.13 . Thus, we are given two objects $X_{12}, X_{34}$ of $\mathscr{O}_{\kappa}$ indexed by the elements of $\odot=\{12,34\}$.

Recall that $B_{\infty}=\mathrm{C}[t]$.

Lemma 17.16. Let $\nabla, \nabla^{\prime}: \mathbf{C}[t] \otimes X_{12} \otimes X_{34} \rightarrow \mathbf{C}[t] \otimes X_{12} \otimes X_{34}$ be the C-linear maps defined by

$$
\begin{aligned}
& \nabla(a \otimes x \otimes y)=t \frac{\partial a}{\partial t} x \otimes y+a L_{0}(x) \otimes y, \\
& \nabla^{\prime}(a \otimes x \otimes y)=t \frac{\partial a}{\partial t} x \otimes y+a x \otimes L_{0}(y),
\end{aligned}
$$

for $a \in \mathbf{C}[t], x \in X_{12}, y \in X_{34}$. Then $\nabla, \nabla^{\prime}$ leave stable the subspace $\Delta_{2, \infty}\left(\mathbf{C}[t] \otimes X_{12} \otimes X_{34}\right) \mathbf{C}[t] \otimes X_{12} \otimes X_{34}$ (see 15.13) and hence induce $\mathbf{C}$-linear maps (which actually coincide) of $\left(\mathbf{C}[t] \otimes X_{12} \otimes X_{34}\right)_{\Delta_{2, \infty}}$ into itself. This map, 
denoted $\nabla_{t \partial} / \partial t$, defines on this last $\mathbf{C}[t]$-module a connection with a regular singularity at 0 .

The connection on $\left(\mathbf{C}[t] \otimes X_{12} \otimes X_{34}\right)_{\Delta_{2, \infty}}$ could be constructed by arguments parallel to those in 17.7-17.14. We prefer, however, to give a more direct definition.

Let $x \in X_{12}, y \in X_{34}$. We have

$$
\begin{aligned}
& \nabla\left(\left(p^{n} c\right)(x \otimes y)\right) \\
& \quad=\nabla\left(\left(\epsilon^{-n} c\right) x \otimes y+t^{n} x \otimes\left(\epsilon^{n} c\right) y\right) \\
& \quad=L_{0}\left(\left(\epsilon^{-n} c\right) x\right) \otimes y+n t^{n} x \otimes\left(\epsilon^{n} c\right) y+t^{n} L_{0}(x) \otimes\left(\epsilon^{n} c\right) y \\
& \quad=\left(\epsilon^{-n} c\right) L_{0} x \otimes y+n\left(\epsilon^{-n} c\right) x \otimes y+n t^{n} x \otimes\left(\epsilon^{n} c\right) y+t^{n} L_{0}(x) \otimes\left(\epsilon^{n} c\right) y \\
& \quad=\left(p^{n} c\right)\left(\left(L_{0}+n\right) x \otimes y\right), \\
& \nabla\left(\left(q^{n} c\right)(x \otimes y)\right) \\
& \quad=\nabla\left(t^{n}\left(\epsilon^{n} c\right) x \otimes y+x \otimes\left(\epsilon^{-n} c\right) y\right) \\
& \quad=n t^{n}\left(\epsilon^{n} c\right) x \otimes y+t^{n} L_{0}\left(\left(\epsilon^{n} c\right) x\right) \otimes y+L_{0}(x) \otimes\left(\epsilon^{-n} c\right) y \\
& \quad=n t^{n}\left(\epsilon^{n} c\right) x \otimes y+t^{n}\left(\epsilon^{n} c\right) L_{0} x \otimes y+-n t^{n}\left(\epsilon^{n} c\right) x \otimes y+L_{0}(x) \otimes\left(\epsilon^{-n} c\right) y \\
& \quad=\left(q^{n} c\right)\left(L_{0} x \otimes y\right) .
\end{aligned}
$$

Since $\left(p^{n} c\right),\left(q^{n} c\right)$ generate $\Delta_{2, \infty}$ as a $\mathbf{C}[t]$-module, we see that $\nabla$ leaves stable the subspace $\Delta_{2, \infty}\left(\mathbf{C}[t] \otimes X_{12}^{\infty} \otimes X_{34}\right)$.

We now show that

$$
\nabla(x \otimes y)-\nabla^{\prime}(x \otimes y) \in \Delta_{2, \infty}\left(\mathbf{C}[t] \otimes X_{12} \otimes X_{34}\right)
$$

for any $x \in X_{12}, y \in X_{34}$.

We can assume that $x \in X_{12}(N), y \in X_{34}(N)$ for some $N \geq 1$. Then

$$
\begin{aligned}
& \nabla(x \otimes y)=\frac{1}{2 \kappa} \sum_{j=0}^{N-1} \sum_{p} l_{j}\left(\epsilon^{-j} c_{p}\right)\left(\epsilon^{j} c_{p}\right) x \otimes y, \\
& \nabla^{\prime}(x \otimes y)=\frac{1}{2 \kappa} \sum_{j=0}^{N-1} \sum_{p} l_{j} x \otimes\left(\epsilon^{-j} c_{p}\right)\left(\epsilon^{j} c_{p}\right) y
\end{aligned}
$$

where $c_{p} \in \mathbf{g}$ are as in 1.14 and $l_{0}=1, l_{j}=2$ for $j>0$.

For any $p$ and any $j \geq 0$ we have

hence,

$$
\begin{aligned}
\left(p^{j} c_{p}\right) & \left(\left(\epsilon^{j} c_{p}\right) x \otimes y\right)-\left(q^{j} c_{p}\right)\left(x \otimes\left(\epsilon^{j} c_{p}\right) y\right. \\
= & \left(\epsilon^{-j} c_{p}\right)\left(\epsilon^{j} c_{p}\right) x \otimes y+t^{j}\left(\epsilon^{j} c_{p}\right) x \otimes\left(\epsilon^{j} c_{p}\right) y \\
& -t^{j}\left(\epsilon^{j} c_{p}\right) x \otimes\left(\epsilon^{j} c_{p}\right) y-x \otimes\left(\epsilon^{-j} c_{p}\right)\left(\epsilon^{j} c_{p}\right) y \\
= & \left(\epsilon^{-j} c_{p}\right)\left(\epsilon^{j} c_{p}\right) x \otimes y-x \otimes\left(\epsilon^{-j} c_{p}\right)\left(\epsilon^{j} c_{p}\right) y ;
\end{aligned}
$$

$$
\left(\epsilon^{-j} c_{p}\right)\left(\epsilon^{j} c_{p}\right) x \otimes y-x \otimes\left(\epsilon^{-j} c_{p}\right)\left(\epsilon^{j} c_{p}\right) y \in \Delta_{2, \infty}\left(\mathbf{C}[t] \otimes X_{12} \otimes X_{34}\right)
$$

and (c) follows. The lemma is proved. 
17.17. We shall apply the previous lemma in the case where $X_{12}=V_{1} \dot{\otimes} V_{2}$, $X_{34}=V_{3} \dot{\otimes} V_{4}$.

We summarize our results as follows:

The $\mathbf{C}[t]$-module $\overline{\mathscr{M}}=\left(\mathbf{C}[t] \otimes\left(V_{1} \dot{\otimes} V_{2}\right) \otimes\left(V_{3} \dot{\otimes} V_{4}\right)\right)_{\Delta_{2, \infty}}$ has a natural connection with a regular singularity at 0 .

For any integer $n \geq 1$, this induces a connection (in the usual sense) on the $\mathrm{C}[t] /\left(t^{n}\right)$-module

$\overline{\mathscr{M}}_{n}=\mathbf{C}[t] /\left(t^{n}\right) \otimes_{\mathbf{C}[t]} \overline{\mathscr{M}}=\overline{\mathscr{M}} / t^{n} \overline{\mathscr{M}}=\left(\mathbf{C}[t] /\left(t^{n}\right) \otimes\left(V_{1} \dot{\otimes} V_{2}\right) \otimes\left(V_{3} \dot{\otimes} V_{4}\right)\right)_{\Delta_{2, n}}$.

This connection makes the following diagram commutative:

(a)

$$
\left(V_{1} \dot{\otimes} V_{2}\right) \otimes\left(V_{3} \dot{\otimes} V_{4}\right) \stackrel{\overline{\mathscr{H}}}{\longrightarrow}\left(V_{1} \dot{\otimes} V_{2}\right) \otimes\left(V_{3} \dot{\otimes} V_{4}\right)
$$

a)

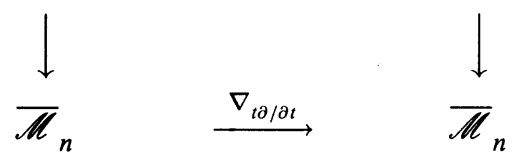

where the vertical maps are the obvious ones and $\overline{\mathscr{H}}$ is the $\mathrm{C}$-linear map defined by

$$
\overline{\mathscr{H}}\left(x \otimes x^{\prime}\right)=x \otimes L_{0}\left(x^{\prime}\right)
$$

for all $x \in V_{1} \dot{\otimes} V_{2}$ and $x^{\prime} \in V_{3} \dot{\otimes} V_{4}$.

17.18. Recall that for $1 \leq n<\infty$ we have an isomorphism

$$
\Phi_{n}: T\left(W_{n}\right)_{\Delta_{2, n}}^{\sharp} \cong W_{n} / \Delta_{4, n} W_{n}=\mathscr{M}_{n} ;
$$

see 15.27 .

By 15.20(a), we have an isomorphism

$$
\Psi_{n}: \overline{\mathscr{M}}_{n}=\left(B_{n} \otimes\left(V_{1} \dot{\otimes} V_{2}\right) \otimes\left(V_{3} \dot{\otimes} V_{4}\right)\right)_{\Delta_{2, n}} \cong T\left(W_{n}\right)_{\Delta_{2, n}}^{\sharp} .
$$

Combining these two isomorphisms, we get an isomorphism of $B_{n}$-modules

$$
\Phi_{n} \Psi_{n}: \overline{\mathscr{M}}_{n} \cong \mathscr{M}_{n}
$$

defined for $1 \leq n<\infty$.

Proposition 17.19. The isomorphism $\Phi_{n} \Psi_{n}$ in 17.18(a) is compatible with the connections on the $B_{n}$-modules $\overline{\mathscr{M}}_{n}, \mathscr{M}_{n}$ defined in 17.14, 17.17.

The proof will be given in 17.20-17.23. The isomorphism 17.18 (a) can be decomposed as a product of four isomorphisms, and we will analyze the compatibility of each of these four isomorphisms with connections.

17.20. Under the natural isomorphism

$$
\Psi_{n}: \overline{\mathscr{M}}_{n} \cong T\left(W_{n}\right)_{\Delta_{2, n}}^{\sharp}
$$

the connection on the $B_{n}$-module $\overline{\mathscr{M}}_{n}$ described in 17.17 becomes a connection $\nabla_{t \partial / \partial t}$ on the $B_{n}$-module $T\left(W_{n}\right)_{\Delta_{2, n}}^{\sharp}$. 
This connection makes the following diagram commutative:

(a)

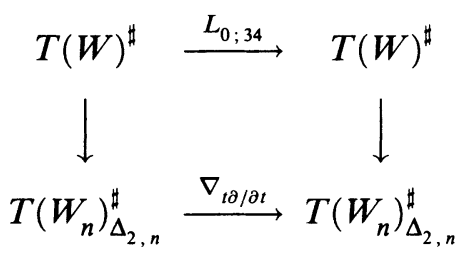

where the vertical maps come from 8.12 (base change $\mathbf{C} \rightarrow B_{n}$ ) and $L_{0 ; 34}$ is, as in 1.15 , the Sugawara operator $L_{0}$ on $T(W)^{\sharp}$, regarded as a $\widetilde{\mathbf{g}}$-module via the imbedding $\delta_{34}: \widetilde{\mathbf{g}} \rightarrow \widetilde{\mathbf{g}}^{\odot}$. (This follows from the obvious fact, that under the natural isomorphism of $\widetilde{\mathbf{g}}^{\odot}$-modules $\left(V_{1} \dot{\otimes} V_{2}\right) \otimes\left(V_{3} \dot{\otimes} V_{4}\right) \cong T(W)^{\sharp}$, the operator $\overline{\mathscr{H}}$ corresponds to the operator $L_{0 ; 34}$.)

17.21. Consider now, for an integer $n \geq 1$, the isomorphism of $B_{n}$-modules

$$
T\left(W_{n}\right)_{\Delta_{2, n}}^{\sharp} \cong\left(T\left(W_{n}\right) \otimes_{B_{n}} X_{n}\right)_{\widetilde{\mathbf{g}}_{B_{n}}^{\odot}}
$$

(see 16.16).

Via this isomorphism, the connection on the $B_{n}$-module $T\left(W_{n}\right)_{\Delta_{2, n}}^{\sharp}$ defined in 17.20 becomes a connection $\nabla_{t a / \partial t}$ on the $B_{n}$-module $\left(T\left(W_{n}\right) \otimes_{B_{n}} X_{n}\right)_{\widetilde{\mathbf{g}}_{B_{n}}^{\mathcal{O}}}$.

From the definitions, this connection makes the following diagram commutative:

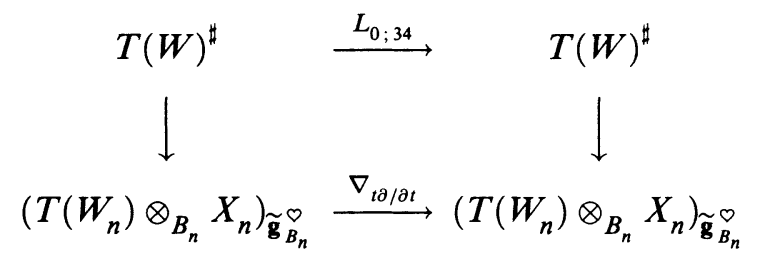

where $L_{0,34}$ is as in 17.20 and the vertical maps are defined by the composition

$$
T(W)^{\sharp} \rightarrow T\left(W_{n}\right)^{\sharp} \rightarrow T\left(W_{n}\right) \otimes_{B_{n}} X_{n} \rightarrow\left(T\left(W_{n}\right) \otimes_{B_{n}} X_{n}\right)_{\widetilde{\mathbf{B}}_{B_{n}}}
$$

(the first map comes from base change (8.12), the second map is $w \mapsto w \otimes 1$, and the third map is obvious).

We now consider, for any $w^{\prime} \in T\left(W_{n}\right)^{\sharp}$ and $y \in X_{n}$, the images of the elements $L_{0,34}\left(w^{\prime}\right) \otimes y$ and $w^{\prime} \otimes L_{0,34}(y)$ of $T\left(W_{n}\right) \otimes_{B_{n}} X_{n} \quad$ in $\left(T\left(W_{n}\right) \otimes_{B_{n}} X_{n}\right)_{\widetilde{\mathbf{B}}_{B_{n}}^{\odot}}$. (Here, $L_{0,34}$ is defined in the same way as in 17.20.)

These two images coincide: we apply 11.12 for the two $\widetilde{\mathbf{g}}_{B_{n}}^{\odot}$-modules $T\left(W_{n}\right)^{\sharp}$ and $X_{n}$, for $t=34 \in \boldsymbol{\phi}=\varnothing$, (see 11.1) and $S=\left\{s_{1}, s_{0}\right\}$; for $k=0$; and for the C-algebra homomorphism $A \rightarrow B_{n}$ which is composition of the homomorphism $A \rightarrow \mathbf{C}$ (evaluation at $\gamma_{s_{1}}, \gamma_{s_{0}}$ as in 10.18) with the obvious homomorphism $\mathbf{C} \rightarrow B_{n}$. (The sum in 11.12 is in this case given by 10.18.) 
Hence the commutativity of the diagram above implies the commutativity of the diagram

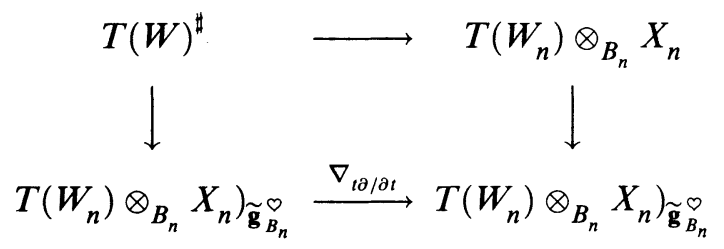

where the upper horizontal map is the composition

$$
T(W)^{\sharp} \rightarrow T\left(W_{n}\right)^{\sharp} \rightarrow T\left(W_{n}\right) \otimes_{B_{n}} X_{n}
$$

(the first map comes from base change (8.12) and the second map is $w \mapsto w \otimes 1$ ), the left vertical map is as in (a) and the right vertical map is the composition

$$
T\left(W_{n}\right)^{\sharp} \otimes_{B_{n}} X_{n} \stackrel{1 \otimes L_{0 ; 34}}{\longrightarrow} T\left(W_{n}\right)^{\sharp} \otimes_{B_{n}} X_{n} \rightarrow\left(T\left(W_{n}\right)^{\sharp} \otimes_{B_{n}} X_{n}\right)_{\widetilde{\mathbf{g}}_{B_{n}}^{\odot}} .
$$

17.22. Consider now, for an integer $n \geq 1$, the isomorphism of $B_{n}$-modules

$$
\left(W_{n}\right)_{\Delta_{4, n}} \rightarrow\left(W_{n} \otimes_{B_{n}} X_{n}\right)_{\Gamma_{B_{n}}}
$$

(see 16.14).

Via this isomorphism, the connection on the $B_{n}$-module $\left(W_{n}\right)_{\Delta_{4, n}}$ defined in 17.14 becomes a connection $\nabla_{t \partial / \partial t}$ on the $B_{n}$-module $\left(W_{n} \otimes_{B_{n}} X_{n}\right)_{\Gamma_{B_{n}}}$.

From the definitions, this connection makes the following diagram commutative:

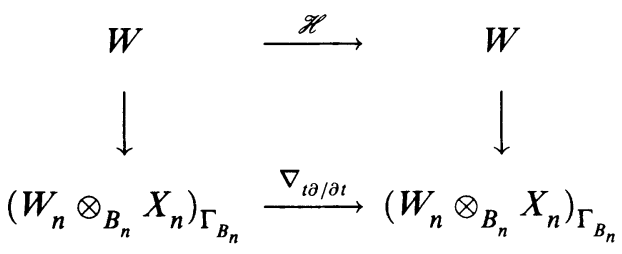

where the vertical maps are defined by the composition

$$
W \rightarrow W_{n} \rightarrow W_{n} \otimes_{B_{n}} X_{n} \rightarrow\left(W_{n} \otimes_{B_{n}} X_{n}\right)_{\Gamma_{B_{n}}}
$$

(the first and third map are the obvious ones, and the second map is $w \mapsto w \otimes 1$ ).

We now consider, for any vectors $x, y, z$ in the $\widetilde{\mathbf{g}}_{B_{n}}^{\widetilde{O}}$-modules

$$
B_{n} \otimes V_{1} \otimes V_{3}, B_{n} \otimes V_{2} \otimes V_{4}, X_{n},
$$

the image of the element

$L_{-1 ; 34} x \otimes y \otimes z-L_{0, ; 34} x \otimes y \otimes z+x \otimes L_{1 ; 34} y \otimes z-x \otimes L_{0 ; 34} y \otimes z+x \otimes y \otimes L_{0 ; 34} z$ of $W_{n} \otimes_{B_{n}} X_{n}$ in $\left(W_{n} \otimes_{B_{n}} X_{n}\right)_{\Gamma_{B_{n}}}$. (Here, as in $1.15, L_{j ; 34}$ are the Sugawara operators in the restriction of these three modules to $\widetilde{\mathbf{g}}_{B_{n}}$ under $\delta_{34}$ : $\left.\widetilde{\mathbf{g}}_{B_{n}} \rightarrow \widetilde{\mathbf{g}}_{B_{n}}{ }^{\circ}\right)$ 
We assert that this image is zero. This follows from 11.12 applied for the three modules above, for $t=34 \in \mathbf{\phi}=\varnothing$ (see 11.1) and $S=\left\{s_{1}, s_{2}, s_{0}\right\}$, for $k=0$, and for the C-algebra homomorphism $A \rightarrow B_{n}$ which is composition of the homomorphism $A \rightarrow \mathbf{C}$ (evaluation at $\gamma_{s_{1}}, \gamma_{s_{2}}, \gamma_{s_{0}}$ as in 10.17) with the obvious homomorphism $\mathbf{C} \rightarrow B_{n}$. (The sum in 11.12 is in this case given by 10.17(a).)

It follows that, for any $w \in W$, the elements $\mathscr{H}(w) \otimes 1$ and $w \otimes L_{0,34}(1)$ of $W_{n} \otimes_{B_{n}} X_{n}$ have the same image in $\left(W_{n} \otimes_{B_{n}} X_{n}\right)_{\Gamma_{B_{n}}}$.

Hence the commutativity of the diagram above implies the commutativity of the diagram

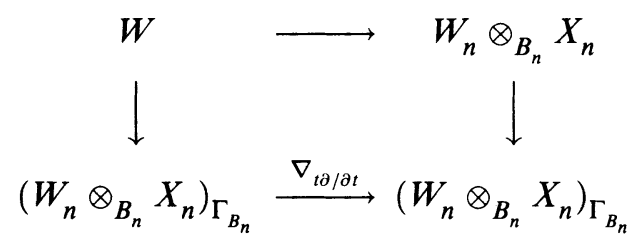

where the upper horizontal map is $w \mapsto w \otimes 1$, the left vertical map is as before, and the right vertical map is the composition

$$
W_{n} \otimes_{B_{n}} X_{n} \stackrel{1 \otimes L_{0 ; 34}}{\longrightarrow} W_{n} \otimes_{B_{n}} X_{n} \rightarrow\left(W_{n} \otimes_{B_{n}} X_{n}\right)_{\Gamma_{B_{n}}}
$$

17.23. We want to show that the isomorphism of $B_{n}$-modules

$$
\Pi_{n}:\left(T\left(W_{n}\right) \otimes_{B_{n}} X_{n}\right)_{\widetilde{\mathbf{g}}_{B_{n}}^{\odot}} \cong\left(W_{n} \otimes_{B_{n}} X_{n}\right)_{\Gamma_{B_{n}}}
$$

(see $16.17(\mathrm{a}))$ is compatible with the connections $\nabla_{t \partial / \partial t}$ on

$$
\left(T\left(W_{n}\right)^{\sharp} \otimes_{B_{n}} X_{n}\right)_{\widetilde{\mathbf{g}}_{B_{n}}^{\widetilde{O}}}, \quad\left(W_{n} \otimes_{B_{n}} X_{n}\right)_{\Gamma_{B_{n}}}
$$

defined in $17.21,17.22$.

Consider the following two compositions:

$$
\begin{gathered}
T(W)^{\sharp} \stackrel{\lambda}{\longrightarrow}\left(T\left(W_{n}\right) \otimes_{B_{n}} X_{n}\right)_{\widetilde{\mathbf{g}}_{B_{n}}^{\odot}} \stackrel{\nabla_{t \partial / \partial t}}{\longrightarrow}\left(T\left(W_{n}\right) \otimes_{B_{n}} X_{n}\right)_{\widetilde{\mathbf{g}}_{B_{n}}^{\odot}} \stackrel{\Pi_{n}}{\longrightarrow}\left(W_{n} \otimes_{B_{n}} X_{n}\right)_{\Gamma_{B_{n}}}, \\
T(W)^{\sharp} \stackrel{\lambda}{\longrightarrow}\left(T\left(W_{n}\right) \otimes_{B_{n}} X_{n}\right)_{\widetilde{\mathbf{g}}_{B_{n}}^{\odot}} \stackrel{\Pi_{n}}{\longrightarrow}\left(W_{n} \otimes_{B_{n}} X_{n}\right)_{\Gamma_{B_{n}}} \stackrel{\nabla_{t \partial / \partial t}}{\longrightarrow}\left(W_{n} \otimes_{B_{n}} X_{n}\right)_{\Gamma_{B_{n}}},
\end{gathered}
$$

where $\lambda: T(W)^{\sharp} \rightarrow\left(T\left(W_{n}\right) \otimes_{B_{n}} X_{n}\right)_{\widetilde{\mathbf{g}}_{B_{n}}^{\odot}}$ is as in $17.21(\mathrm{~b})$.

Let $w \in T(W)^{\sharp}$. We regard $w$ as an element in the inverse limit $\widehat{W}$; thus, we may represent $w$ by a sequence $\left(w_{1}, w_{2}, w_{3}, \ldots\right)$ of compatible elements of $W$. The image of $w$ in $T\left(W_{n}\right)^{\sharp}$ is denoted $j(w)$; it can be represented by the same sequence $\left(w_{1}, w_{2}, w_{3}, \ldots\right)$ regarded now as a sequence of elements in $W_{n}$. By the commutative diagram $17.21(\mathrm{~b})$ we have that $\nabla_{t \partial / \partial t}(\lambda(w))$ is the image of $j(w) \otimes L_{0 ; 34}(1)$ in $\left(T\left(W_{n}\right) \otimes_{B_{n}} X_{n}\right)_{\widetilde{\mathbf{g}}_{B_{n}}^{\odot}}$. Hence, by the definition of $\Pi_{n}$, we have that $\Pi_{n}\left(\nabla_{t \partial / \partial t}(\lambda(w))\right)$ is equal to the image of $w_{k} \otimes L_{0 ; 34}(1)$ in $\left(W_{n} \otimes_{B_{n}} X_{n}\right)_{\Gamma_{B_{n}}}$ for sufficiently large $k$. 
Again, by the definition of $\Pi_{n}$, we have that $\Pi_{n}((\lambda(w))$ is equal to the image $w_{k} \otimes 1$ in $\left(W_{n} \otimes_{B_{n}} X_{n}\right)_{\Gamma_{B_{n}}}$ for sufficiently large $k$. Using the commutative diagram 17.22(b), it follows that $\nabla_{t \partial / \partial t}\left(\Pi_{n}((\lambda(w)))\right.$ is equal to the image of $w_{k} \otimes L_{0 ; 34}(1)$ in $\left(W_{n} \otimes_{B_{n}} X_{n}\right)_{\Gamma_{B_{n}}}$ for sufficiently large $k$.

Thus, we have the equality of compositions $\nabla_{t \partial / \partial t} \Pi_{n} \lambda=\Pi_{n} \nabla_{t \partial / \partial t} \lambda$.

Now the image of $\lambda$ generates $\left(T\left(W_{n}\right) \otimes_{B_{n}} X_{n}\right)_{\widetilde{\mathbf{g}}_{B_{n}}^{\circ}}$ as a $B_{n}$-module. (Indeed, $\lambda$ is the composition of $T(W)^{\sharp} \rightarrow T\left(W_{n}\right)^{\sharp}$ with a surjective homomorphism of $B_{n}$-modules $T\left(W_{n}\right)^{\sharp} \rightarrow\left(T\left(W_{n}\right) \otimes_{B_{n}} X_{n}\right)_{\widetilde{\mathbf{B}}_{B_{n}}^{\circ}}$, and the image of $T(W)^{\sharp} \rightarrow T\left(W_{n}\right)^{\sharp}$ generates $T\left(W_{n}\right)^{\sharp}$ by 8.12 .)

Moreover, both $\Pi_{n}^{-1} \nabla_{t \partial / \partial t} \Pi_{n}$ and $\nabla_{t \partial / \partial t}$ are connections on the $B_{n}$-module $\left(T\left(W_{n}\right) \otimes_{B_{n}} X_{n}\right)_{\widetilde{\mathbf{g}}_{B_{n}}^{\circ}}$; hence, their difference is a $B_{n}$-linear map. Hence, the equality

$$
\left(\Pi_{n}^{-1} \nabla_{t \partial / \partial t} \Pi_{n}-\nabla_{t \partial / \partial t}\right) \lambda=0
$$

implies the equality

$$
\Pi_{n}^{-1} \nabla_{t \partial / \partial t} \Pi_{n}-\nabla_{t \partial / \partial t}=0
$$

Thus the two connections $\nabla_{t \partial / \partial t}$ on $\left(T\left(W_{n}\right) \otimes_{B_{n}} X_{n}\right)_{\widetilde{\mathbf{B}}_{B_{n}}^{\odot}}$ and $\left(W_{n} \otimes_{B_{n}} X_{n}\right)_{\Gamma_{B_{n}}}$ correspond to each other under the isomorphism $\Pi_{n}$, as asserted.

We have therefore verified that each of the four isomorphisms of which $17.18(a)$ is the composition is connection preserving. Hence the isomorphism 17.18(a) itself is connection preserving. This completes the proof of Proposition 17.19 .

17.24. For any finitely generated $A_{\infty}$-module $M$ with torsion module $\tau(M)$ annihilated by a power of $t$, we set $M^{f}=M / \tau(M)$. Then $M^{f}$ is a finitely generated free $A_{\infty}$-module. We also set $\widehat{M}=\lim _{n \geq 1} M / t^{n} M$. This is a finitely generated $\mathbf{C}[[t]]$-module. We have an exact sequence

$$
0 \rightarrow \tau(M) \rightarrow \widehat{M} \rightarrow \widehat{M^{f}} \rightarrow 0 .
$$

Consider now the $A_{\infty}$-modules $\mathscr{M}$ (see 17.10) and $\mathscr{M}^{\prime}=A_{\infty} \otimes_{\mathbf{C}[t]} \overline{\mathscr{M}}$, where $\overline{\mathscr{M}}$ is as in 17.17 and $A_{\infty}$ is regarded as a $\mathrm{C}[t]$-algebra via the obvious imbedding $\mathbf{C}[t] \subset \mathbf{C}\left[t,(t-1)^{-1}\right]=A_{\infty}$. Then $\mathscr{M}, \mathscr{M}^{\prime}$ are finitely generated $A_{\infty}$-modules with torsion annihilated by a power of $t$. For any $n \geq 1$, we consider the isomorphism

$$
\mathscr{M}^{\prime}\left|t^{n} \mathscr{M}^{\prime}=\overline{\mathscr{M}}\right| t^{n} \overline{\mathscr{M}} \cong \mathscr{M} / t^{n} \mathscr{M}
$$

given by 15.27 .

When $n$ varies, the isomorphisms (b) are compatible with the maps in the standard projective system

$$
\mathscr{M}^{\prime} / t \mathscr{M}^{\prime} \leftarrow \mathscr{M}^{\prime} / t^{2} \mathscr{M}^{\prime} \leftarrow \cdots
$$


and with the maps in the analogous projective system for $\mathscr{M}$ (see 15.26). Hence they induce an isomorphism of $\mathbf{C}[[t]]$-modules

$$
\widehat{\mathscr{M}^{\prime}} \cong \widehat{\mathscr{M}}
$$

This restricts to an isomorphism $\tau(M) \cong \tau\left(M^{\prime}\right)$ and defines by passage to quotient an isomorphism

$$
\widehat{\mathscr{M}^{\prime}} \cong \widehat{\mathscr{M}^{f}}
$$

(see (a)).

This induces for any $n \geq 1$ an isomorphism

$$
\mathscr{M}^{\prime f} / t^{n} \mathscr{M}^{\prime f} \cong \mathscr{M}^{f} / t^{n} \mathscr{M}^{f}
$$

which is compatible with the isomorphism (b).

Let $\mathbf{M}=\operatorname{Hom}_{A_{\infty}}\left(\mathscr{M}^{\prime f}, \mathscr{M}^{f}\right)$. Then $\mathbf{M}$ is a finitely generated free $A_{\infty}$ module and we have canonically

$$
\widehat{\mathbf{M}}=\operatorname{Hom}_{\mathbf{C}[t]]}\left(\widehat{\mathscr{M}^{\prime f}}, \widehat{\mathscr{M}^{f}}\right) .
$$

Thus, the isomorphism (d) may be regarded as an element $\omega \in \widehat{\mathbf{M}}$.

17.25. Recall $(17.14,17.17)$ that the $A_{\infty}$-module $\mathscr{M}$ and the $\mathrm{C}[t]$-module $\overline{\mathscr{M}}$ have natural connections with regular singularities at 0 . Then the $A_{\infty}$-module $\mathscr{M}^{\prime}$ inherits from $\overline{\mathscr{M}}$ a connection with a regular singularity at 0 . The connection operator $\nabla_{t \partial / \partial t}$ on $\mathscr{M}$ (resp. $\mathscr{M}^{\prime}$ ) clearly leaves stable the torsion submodule and, hence, induces a connection with a regular singularity at 0 on the corresponding quotient $\mathscr{M}^{f}$ (resp. $\mathscr{M}^{\prime f}$ ).

Let $\nabla_{t \partial / \partial t}: \mathbf{M} \rightarrow \mathbf{M}$ be the $\mathbf{C}$-linear map $f \mapsto \nabla_{t \partial / \partial t} f$ where

$$
\left(\nabla_{t \partial / \partial t} f\right)\left(m^{\prime}\right)=\nabla_{t \partial / \partial t}\left(f\left(m^{\prime}\right)\right)-f\left(\nabla_{t \partial / \partial t} m^{\prime}\right)
$$

for all $m^{\prime} \in \mathscr{M}^{\prime f}$. It is clear that this operator defines a connection with a regular singularity at 0 on $\mathbf{M}$.

This operator leaves stable the submodules $t^{n} \mathbf{M}$ of $\mathbf{M}$; hence, on the one hand, it extends naturally to an operator $\nabla_{t \partial / \partial t}: \widehat{\mathbf{M}} \rightarrow \widehat{\mathbf{M}}$, and, on the other hand, it induces a connection on the quotient $\mathbf{C}[t] /\left(t^{n}\right)$-module

$$
\mathbf{M} / t^{n} \mathbf{M}=\operatorname{Hom}_{\mathbf{C}[t] /\left(t^{n}\right)}\left(\mathscr{M}^{\prime f} / t^{n} \mathscr{M}^{f}, \mathscr{M}^{f} / t^{n} \mathscr{M}^{f}\right)
$$

for any integer $n \geq 1$.

17.26. Now the free $A_{\infty}$-modules $\mathscr{M}^{f}, \mathscr{M}^{\prime f}, \mathbf{M}$ are spaces of sections of welldefined algebraic vector bundles on $\mathbf{C}-\{1\}$. The restrictions of these vector bundles to the real interval $(-\infty, 1)$ will be regarded as real analytic vector bundles and will be denoted by $\mathscr{M}_{a n}, \mathscr{M}_{a n}^{\prime}, \mathbf{M}_{a n}$.

These three vector bundles have natural connections with regular singularity at 0 . 
17.27. For any integer $n \geq 1$, the connection operator of the $\mathbf{C}[t] /\left(t^{n}\right)$-module $\mathscr{M} / t^{n} \mathscr{M}$ (resp. $\overline{\mathscr{M}} / t^{n} \overline{\mathscr{M}}=\mathscr{M}^{\prime} / t^{n} \mathscr{M}^{\prime}$ ) leaves stable the image of $\tau(\mathscr{M})$ (resp. $\left.\tau\left(\mathscr{M}^{\prime}\right)\right)$ and, hence, induces a connection on the quotient $\mathbf{C}[t] /\left(t^{n}\right)$-module $\mathscr{M}^{f} / t^{n} \mathscr{M}^{f}$ (resp. $\mathscr{M}^{\prime f} / t^{n} \mathscr{M}^{\prime f}$ ).

These connections induce a connection on the $\mathbf{C}[t] /\left(t^{n}\right)$-module

$$
\operatorname{Hom}_{\mathbf{C}[t] /\left(t^{n}\right)}\left(\mathscr{M}^{\prime f} / t^{n} \mathscr{M}^{\prime f}, \mathscr{M}^{f} / t^{n} \mathscr{M}^{f}\right)=\mathbf{M} / t^{n} \mathbf{M}
$$

formally as in $17.25(a)$. One checks that this coincides with the connection defined at the end of 17.25 .

The isomorphism 17.24(e) can be interpreted as an element $\omega_{n} \in \mathbf{M} / t^{n} \mathbf{M}$ (see $(\mathrm{a}))$.

It is clear that $\omega_{n}$ is the image of $\omega \in \widehat{\mathbf{M}}$ (see 17.24) under the canonical $\operatorname{map} \widehat{\mathbf{M}} \rightarrow \mathbf{M} / t^{n} \mathbf{M}$.

It follows from Proposition 15.27 that the connections on $\mathscr{M}^{f} / t^{n} \mathscr{M}^{f}$, $\mathscr{M}^{\prime f} / t^{n} \mathscr{M}^{\prime f}$ correspond to each other under the isomorphism 17.24(e). This means that $\omega_{n}$ is annihilated by the connection operator $\nabla_{t \partial / \partial t}$ of $\mathbf{M} / t^{n} \mathbf{M}$. Since this holds for all $n$, it follows that $\omega$ is annihilated by the operator $\nabla_{t \partial / \partial t}$ of $\widehat{\mathbf{M}}$. In other words, $\omega \in \widehat{\mathbf{M}}$ is a formal solution of the linear differential equation given by the connection $\nabla_{t \partial / \partial t}$ with regular singularity at 0 on the finitely generated free $A_{\infty}$-module $\mathbf{M}$.

By the classical theory of regular singularities for linear differential equations (see [W]), it follows that there is a unique analytic section $\widetilde{\omega}$ of $\mathbf{M}_{a n}$ (defined over $(-\infty, 1)$ which, on the one hand, is annihilated by the connection operator $\nabla_{t \partial / \partial t}$ on this vector bundle and, on the other hand, has power series expansion at 0 given by $\omega$.

17.28. We may regard $\widetilde{\omega}$ as a morphism from $\mathscr{M}_{a n}^{\prime}$ to $\mathscr{M}_{a n}$. By the definition of $\omega$, we see that $\widetilde{\omega}$ is formally (at 0 ) an isomorphism from $\mathscr{M}_{a n}^{\prime}$ to $\mathscr{M}_{a n}$. It follows that $\widetilde{\omega}$ defines an analytic isomorphism from $\mathscr{M}_{a n}^{\prime}$ to $\mathscr{M}_{a n}$ on some interval $(-a, a)$ where $0<a<1$. But $\mathscr{M}_{a n}^{\prime}$ and $\mathscr{M}_{a n}$ are flat vector bundles outside 0 and $\widetilde{\omega}$ preserves these flat structures. It follows that $\widetilde{\omega}$ defines an analytic isomorphism from $\mathscr{M}_{a n}^{\prime}$ to $\mathscr{M}_{a n}$ outside 0 and, therefore, also over the entire interval $(-\infty, 1)$.

In particular, $\widetilde{\omega}$ defines an analytic isomorphism (preserving the flat structures) from $\mathscr{M}_{a n}^{\prime}$ to $\mathscr{M}_{a n}$ over the real interval $(-\infty, 0)$. Hence it defines an isomorphism

$$
\mathscr{S}\left(\mathscr{M}_{a n}^{\prime}\right) \cong \mathscr{S}\left(\mathscr{M}_{a n}\right)
$$

between the spaces of horizontal sections of these vector bundles over $(-\infty, 0)$. Now $\mathscr{S}\left(\mathscr{M}_{a n}^{\prime}\right)$ is canonically isomorphic (via restriction) to the fibre of $\mathscr{M}_{a n}^{\prime}$ at any point of $(-\infty, 0)$ (since this interval is contractible). The fibre at -1 is the specialization of $\mathscr{M}^{\prime}$ for $t=-1$ or, equivalently, the specialization of $\mathscr{\mathscr { M }}$ for $t=-1$, and this is, by definition, $\left\langle V_{1} \dot{\otimes} V_{2}, V_{3} \dot{\otimes} V_{4}\right\rangle$. Thus,

$$
\mathscr{S}\left(\mathscr{M}_{a n}^{\prime}\right)=\left\langle V_{1} \dot{\otimes} V_{2}, V_{3} \dot{\otimes} V_{4}\right\rangle .
$$


Similarly, $\mathscr{S}\left(\mathscr{M}_{a n}\right)$ is canonically isomorphic (via restriction) to the specialization of $\mathscr{M}$ at any point of $(-\infty, 0)$ or, equivalently (see 17.6), to the specialization of the $A$-module $Y / \Delta Y$ at the point $\nu(t) \in \mathscr{V}$ for any $t \in(-\infty, 0)$. (Here, $\nu: \mathbf{C}-\{0,1\} \rightarrow \mathscr{V}$ is as in 17.1, $A$ is the coordinate algebra of $\mathscr{V}$.)

We will now verify (using the definition 17.1(a)) that $\nu(t)$ is contained in the subset $\underline{\mathscr{V}}_{0}$ of $\underline{\mathscr{V}}$ described in 13.1 (for $r=4$ ) provided that $t \in(-\infty, 0)$.

In the following diagram we represent the eight points of $P_{\mathbf{R}}^{1}$ given by $\gamma_{i}(0)$, $\gamma_{i}(\delta)$ where $\gamma_{i}$ are the four automorphisms of $P^{1}$ given in 17.1(a) and $\delta>0$ is small. (The values of $\gamma_{i}(0)$ are given at the four corners of a square as in 17.1, and the values of $\gamma_{i}(\delta)$ are given near the value of $\gamma_{i}(0)$ with the same i.)

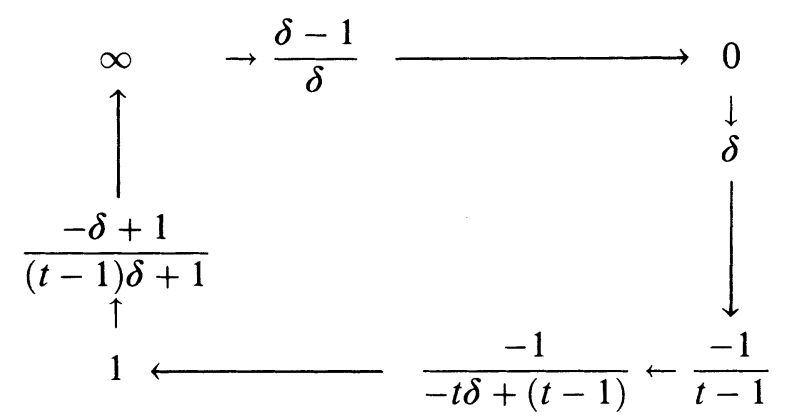

If $t \in(-\infty, 0)$ and $\delta>0$ is sufficiently small (depending on $t$ ), the eight points above cut the circle in the manner shown. This shows that $\nu(t) \in \underline{\mathscr{V}}_{0}$. From the definition in 13.3, we see now that

$$
\mathscr{S}\left(\mathscr{M}_{\text {an }}\right)=\left\langle V_{1}, V_{2}, V_{3}, V_{4}\right\rangle .
$$

We therefore obtain the following result.

Theorem 17.29. The isomorphism $17.28(\mathrm{a})-(\mathrm{c})$ combine to give a canonical isomorphism

$$
\left\langle V_{1} \dot{\otimes} V_{2}, V_{3} \dot{\otimes} V_{4}\right\rangle \cong\left\langle V_{1}, V_{2}, V_{3}, V_{4}\right\rangle .
$$

17.30. The following generalization of the previous theorem can be proved essentially along the same lines:

Given objects $V_{1}, V_{2}, \ldots, V_{r}, V_{r+1}, \ldots, V_{r+r^{\prime}}$ of $\mathscr{O}_{\kappa}$ (where $r, r^{\prime} \geq 1$ ) we have a canonical isomorphism of $\mathbf{C}$-vector spaces

$$
\left\langle V_{1} \dot{\otimes} V_{2} \dot{\otimes} \cdots \dot{\otimes} V_{r}, V_{r+1} \dot{\otimes} \cdots \dot{\otimes} V_{r+r^{\prime}}\right\rangle \cong\left\langle V_{1}, V_{2}, \ldots, V_{r+r^{\prime}}\right\rangle .
$$

(For $r=1$ we interpret $V_{1} \dot{\otimes} \cdots \dot{\otimes} V_{r}$ as $V_{1}$.)

\section{THE ASSOCIATIVITY ISOMORPHISM}

In this section we will complete the construction of the associativity isomorphism, started in $\S 15$.

18.1. Let $V_{1}, V_{2}, V_{3}, V_{4}$ be objects of $\mathscr{O}_{\kappa}$. In the last section we have constructed a canonical isomorphism

$$
\left\langle V_{1} \dot{\otimes} V_{2}, V_{3} \dot{\otimes} V_{4}\right\rangle \cong\left\langle V_{1}, V_{2}, V_{3}, V_{4}\right\rangle .
$$


The same construction applied to $V_{4}, V_{1}, V_{2}, V_{3}$ gives an isomorphism

$$
\left\langle V_{4} \dot{\otimes} V_{1}, V_{2} \dot{\otimes} V_{3}\right\rangle \cong\left\langle V_{4}, V_{1}, V_{2}, V_{3}\right\rangle \text {. }
$$

By 13.3, $\left\langle V_{1}, V_{2}, V_{3}, V_{4}\right\rangle$ depends only on the cyclic order of the $V_{i}$. Thus, we have $\left\langle V_{1}, V_{2}, V_{3}, V_{4}\right\rangle=\left\langle V_{4}, V_{1}, V_{2}, V_{3}\right\rangle$ and, therefore, (a),(b) combine to give an isomorphism

$$
\left\langle V_{1} \dot{\otimes} V_{2}, V_{3} \dot{\otimes} V_{4}\right\rangle \cong\left\langle V_{4} \dot{\otimes} V_{1}, V_{2} \dot{\otimes} V_{3}\right\rangle .
$$

18.2. Recall from 13.5 the canonical isomorphism

$$
\left\langle V \dot{\otimes} V^{\prime}, V^{\prime \prime}\right\rangle \cong\left\langle V, V^{\prime}, V^{\prime \prime}\right\rangle
$$

for any $V, V^{\prime}, V^{\prime \prime}$ in $\mathscr{O}_{\kappa}$.

Using several times $(a)$ and the invariance of $\langle$,$\rangle under cyclic permutation,$ we have

$$
\begin{aligned}
\left\langle V_{1} \dot{\otimes} V_{2}, V_{3} \dot{\otimes} V_{4}\right\rangle & \cong\left\langle V_{3} \dot{\otimes} V_{4}, V_{1} \dot{\otimes} V_{2}\right\rangle \cong\left\langle V_{3}, V_{4}, V_{1} \dot{\otimes} V_{2}\right\rangle \cong\left\langle V_{1} \dot{\otimes} V_{2}, V_{3}, V_{4}\right\rangle \\
& \cong\left\langle\left(V_{1} \dot{\otimes} V_{2}\right) \dot{\otimes} V_{3}, V_{4}\right\rangle, \\
\left\langle V_{4} \dot{\otimes} V_{1}, V_{2} \dot{\otimes} V_{3}\right\rangle & \cong\left\langle V_{4}, V_{1}, V_{2} \dot{\otimes} V_{3}\right\rangle \cong\left\langle V_{1}, V_{2} \dot{\otimes} V_{3}, V_{4}\right\rangle \\
& \cong\left\langle V_{1} \dot{\otimes}\left(V_{2} \dot{\otimes} V_{3}\right), V_{4}\right\rangle .
\end{aligned}
$$

Combining these with $18.1(\mathrm{c})$, we obtain

$$
\left\langle\left(V_{1} \dot{\otimes} V_{2}\right) \dot{\otimes} V_{3}, V_{4}\right\rangle \cong\left\langle V_{1} \dot{\otimes}\left(V_{2} \dot{\otimes} V_{3}\right), V_{4}\right\rangle,
$$

or, equivalently (see $2.32(\mathrm{c}))$,

$$
\operatorname{Hom}_{\mathscr{O}_{\kappa}}\left(\left(V_{1} \dot{\otimes} V_{2}\right) \dot{\otimes} V_{3}, D\left(V_{4}\right)\right) \cong \operatorname{Hom}_{\mathscr{C}_{\kappa}}\left(V_{1} \dot{\otimes}\left(V_{2} \dot{\otimes} V_{3}\right), D\left(V_{4}\right)\right) .
$$

Since this isomorphism is functorial, it must be induced by a well-defined isomorphism in $\mathscr{O}_{\kappa}$ :

$$
\left(V_{1} \dot{\otimes} V_{2}\right) \dot{\otimes} V_{3} \cong V_{1} \dot{\otimes}\left(V_{2} \dot{\otimes} V_{3}\right) .
$$

This isomorphism, which is functorial in all arguments, is called the associativity isomorphism.

\section{APPENDIX: INDUCED MODULES}

In arguments involving induced modules for modules over a Lie algebra, the Poincaré-Birkhoff-Witt theorem is often used. However, when we work over a ring rather than a field, the Poincare-Birkhoff-Witt theorem is not always valid. The results of this appendix are concerned with the question of how to avoid using the Poincaré-Birkhoff-Witt theorem in the study of induced modules.

A.1. Let $A$ be a commutative $\mathbf{C}$-algebra. An induction datum is a triple $\left(\mathbf{h}, \mathbf{h}^{+}\right.$, $V$ ) where $\mathbf{h}$ is an $A$-Lie algebra, $\mathbf{h}^{+}$is an $A$-Lie subalgebra of $\mathbf{h}$, and $V$ is a $\mathbf{h}^{+}$-module. Then

$$
\widetilde{V}=U(\mathbf{h}) \otimes_{U\left(\mathbf{h}^{+}\right)} V
$$

is naturally a $\mathbf{h}$-module, called the induced module.

A split induction datum is a quadruple $\left(\mathbf{h}, \mathbf{h}^{-}, \mathbf{h}^{+}, V\right)$ where $\left(\mathbf{h}, \mathbf{h}^{+}, V\right)$ is an induction datum and $\mathbf{h}^{-}$is an $A$-Lie subalgebra of $\mathbf{h}$ such that $\mathbf{h}=\mathbf{h}^{-} \oplus \mathbf{h}^{+}$ 
as an $A$-module and such that there exists an h-module structure on the $A$ module $U\left(\mathbf{h}^{-}\right) \otimes_{A} V$ with the following property:

$$
\xi(u \otimes v)=(\xi u) \otimes v \quad \text { and } \quad \zeta(1 \otimes v)=1 \otimes \zeta v
$$

for all $\xi \in \mathbf{h}^{-}, \zeta \in \mathbf{h}^{+}, u \in U\left(\mathbf{h}^{-}\right), v \in V$. (The h-module structure in (a) is necessarily unique.) We shall denote the $\mathbf{h}$-module $U\left(\mathbf{h}^{-}\right) \otimes_{A} V$ by $V^{b}$.

Lemma A.2. (a) Assume that $\left(\mathbf{h}, \mathbf{h}^{+}, V\right)$ is an induction datum and that $\mathbf{h}^{-}$is an A-Lie subalgebra of $\mathbf{h}$ such that $\mathbf{h}=\mathbf{h}^{+} \oplus \mathbf{h}^{-}$as A-modules. Assume also that $\mathbf{h}^{+}, \mathbf{h}^{-}$are free as A-modules. Then $\left(\mathbf{h}, \mathbf{h}^{-}, \mathbf{h}^{+}, V\right)$ is a split induction datum and the h-module homomorphism $\widetilde{V} \rightarrow V^{\mathrm{b}}$ given by $u \otimes v \mapsto u(1 \otimes v)$ for all $u \in U(\mathbf{h})$ and $v \in V$ is an isomorphism.

(b) Assume that $\left(\mathbf{h}, \mathbf{h}^{-}, \mathbf{h}^{+}, V\right)$ is a split induction datum (but we make no freeness assumptions). Then the h-module homomorphism $\widetilde{V} \rightarrow V^{b}$ given by $u \otimes v \mapsto u(1 \otimes v)$ for all $u \in U(\mathbf{h})$ and $v \in V$ is an isomorphism.

Under the hypothesis of (a), we are allowed to use the Poincaré-Birkhoff-Witt theorem. We see that $U(\mathbf{h})=U\left(\mathbf{h}^{-}\right) \otimes_{B_{n}} U\left(\mathbf{h}^{+}\right)$. It follows that

$$
\tilde{V}=U(\mathbf{h}) \otimes_{U\left(\mathbf{h}^{+}\right)} V=U\left(\mathbf{h}^{-}\right) \otimes_{B_{n}} U\left(\mathbf{h}^{+}\right) \otimes_{U\left(\mathbf{h}^{+}\right)} V=U\left(\mathbf{h}^{-}\right) \otimes_{B_{n}} V
$$

and (a) follows.

We now prove (b).

Let $X$ be any h-module. We have a canonical map

$$
\operatorname{Hom}_{\mathbf{h}}\left(V^{b}, X\right) \rightarrow \operatorname{Hom}_{\mathbf{h}^{+}}(V, X)
$$

defined by taking composition with the natural homomorphism $V \rightarrow V^{b}$, $(v \mapsto 1 \otimes v)$. We construct a map in the opposite direction. Let $f: V \rightarrow X$ be a homomorphism of $\mathbf{h}^{+}$-modules. We define an $A$-linear map $f^{\prime}: V^{b} \rightarrow X^{-}$ by $f^{\prime}\left(\left(\xi_{1} \ldots \xi_{p}\right) v\right)=\xi_{1} \ldots \xi_{p} f(v)$ for any $\xi_{1}, \ldots, \xi_{p} \in \mathbf{h}^{-}$and any $v \in V$; we omit the tensor product sign. It is clear that

$$
f^{\prime}\left(\xi v_{1}\right)=\xi f^{\prime}\left(v_{1}\right) \text { for all } \xi \in \mathbf{h}^{-} \text {and all } v_{1} \in V^{b}
$$

We show by induction on $p \geq 0$ that

$$
f^{\prime}\left(\xi^{\prime}\left(\left(\xi_{1} \cdots \xi_{p}\right) v\right)\right)=\xi^{\prime} f^{\prime}\left(\left(\xi_{1} \cdots \xi_{p}\right) v\right)
$$

for any $\xi_{1}, \ldots, \xi_{p} \in \mathbf{h}^{-}$, any $\xi^{\prime} \in \mathbf{h}^{+}$, and any $v \in V$. This is clear for $p=0$; hence, we may assume that $p \geq 1$.

In the following computation we write $u^{\prime}=\xi_{2} \cdots \xi_{p}, u=\xi_{1} u^{\prime}$; for any element $\eta \in \mathbf{h}$ we write $\eta=\eta^{-}+\eta^{+}$where $\eta^{-} \in \mathbf{h}^{-}$and $\eta^{+} \in \mathbf{h}^{+}$. We have, using (b) and the induction hypothesis,

$$
\begin{aligned}
f^{\prime}\left(\xi^{\prime}(u v)\right) & =f^{\prime}\left(\xi^{\prime} \xi_{1}\left(u^{\prime} v\right)\right) \\
& =f^{\prime}\left(\xi_{1} \xi^{\prime}\left(u^{\prime} v\right)\right)+f^{\prime}\left(\left[\xi^{\prime}, \xi_{1}\right]^{-}\left(u^{\prime} v\right)\right)+f^{\prime}\left(\left[\xi^{\prime}, \xi_{1}\right]^{+}\left(u^{\prime} v\right)\right) \\
& =\xi_{1} f^{\prime}\left(\xi^{\prime}\left(u^{\prime} v\right)\right)+\left[\xi^{\prime}, \xi_{1}\right]^{-} f^{\prime}\left(u^{\prime} v\right)+\left[\xi^{\prime}, \xi_{1}\right]^{+} f^{\prime}\left(u^{\prime} v\right) \\
& =\xi_{1} \xi^{\prime} f^{\prime}\left(u^{\prime} v\right)+\left[\xi^{\prime}, \xi_{1}\right] f^{\prime}\left(u^{\prime} v\right) \\
& =\xi^{\prime} \xi_{1} f^{\prime}\left(u^{\prime} v\right)=\xi^{\prime} f^{\prime}(u v)
\end{aligned}
$$

and (c) is established. 
Thus, we have $f^{\prime} \in \operatorname{Hom}_{\mathbf{h}}\left(V^{b}, X\right)$. It is clear that $f \mapsto f^{\prime}$ is the inverse of the map (a). Hence (a) is an isomorphism.

By the definition of tensor product, we have

$$
\operatorname{Hom}_{\mathbf{h}}\left(U(\mathbf{h}) \otimes_{U\left(\mathbf{h}^{+}\right)} V, X\right) \cong \operatorname{Hom}_{\mathbf{h}^{+}}(V, X) .
$$

Combining with the isomorphism (b) we obtain

$$
\operatorname{Hom}_{\mathbf{h}}\left(U(\mathbf{h}) \otimes_{U\left(\mathbf{h}^{+}\right)} V, X\right) \cong \operatorname{Hom}_{\mathbf{h}}\left(V^{b}, X\right) .
$$

Since this holds for any $X$, the lemma follows.

A.3. If $V$ is a module over an $A$-Lie algebra $\Gamma$, we set $V_{\Gamma}=V / \Gamma V$ (space of coinvariants). An equivalent definition is $V_{\Gamma}=A \otimes_{U(\Gamma)} V$ where $A$ is taken with the zero action of $\Gamma$.

A.4. Let $\mathbf{h}$ be an $A$-Lie algebra, and let $\mathbf{h}^{+}, \mathbf{h}^{-}, \mathbf{h}^{\prime}, \mathbf{h}^{\prime+}, \mathbf{h}^{\prime-}$ be $A$-Lie subalgebras of $\mathbf{h}$ such that $\mathbf{h}=\mathbf{h}^{-} \oplus \mathbf{h}^{+}, \mathbf{h}^{\prime}=\mathbf{h}^{\prime-} \oplus \mathbf{h}^{\prime+}$, as $A$-modules and such that $\mathbf{h}^{-}=\mathbf{h}^{\prime-}$.

Let $V$ be an $\mathbf{h}^{+}$-module and let $W$ be an $\mathbf{h}^{\prime}$-module.

We assume that $\left(\mathbf{h}, \mathbf{h}^{-}, \mathbf{h}^{+}, V\right)$ is a split induction datum; let $V^{\text {b }}$ be the h-module defined as in A.1.

We restrict $V^{b}$ to an $\mathbf{h}^{\prime}$-module and form the tensor product $V^{b} \otimes_{A} W$; this is an $\mathbf{h}^{\prime}$-module in a natural way, and hence $\left(V^{b} \otimes_{A} W\right)_{\mathbf{h}^{\prime}}$ is defined.

We restrict $W$ and $V$ to $\mathbf{h}^{\prime+}$-modules; then $V \otimes_{A} W$ is naturally a $\mathbf{h}^{\prime+}$ module and hence $\left(V \otimes_{A} W\right)_{\mathbf{h}^{\prime+}}$ is defined.

We have a canonical imbedding $l: V \rightarrow V^{b}$ given by $v \rightarrow 1 \otimes v$. Then $l \otimes 1: V \otimes_{A} W \rightarrow V^{b} \otimes_{A} W$ carries $\mathbf{h}^{\prime+}\left(V \otimes_{A} W\right)$ into $\mathbf{h}^{\prime}\left(V^{b} \otimes_{A} W\right)$; hence, we have an induced map

$$
\left(V \otimes_{A} W\right)_{\mathbf{h}^{\prime+}} \rightarrow\left(V^{b} \otimes_{A} W\right)_{\mathbf{h}^{\prime}}
$$

In this set-up, we have the following result.

Proposition A.5. The A-linear map A.4(a) is an isomorphism.

Our assumptions imply that $\left(\mathbf{h}^{\prime}, \mathbf{h}^{\prime-}, \mathbf{h}^{\prime+},\left.V\right|_{\mathbf{h}^{++}}\right)$is a split induction datum and that the corresponding $\mathbf{h}^{\prime}$-module $\left(\left.V\right|_{\mathbf{h}^{+}}\right)^{b}$ (see A.1) is $\left.V^{b}\right|_{\mathbf{h}^{\prime}}$. Hence, if we replace $\left(\mathbf{h}, \mathbf{h}^{+}, \mathbf{h}^{\prime}, \mathbf{h}^{\prime+}, V, W, V^{b}\right)$ by $\left(\mathbf{h}^{\prime}, \mathbf{h}^{\prime+}, \mathbf{h}^{\prime}, \mathbf{h}^{\prime+},\left.V\right|_{\mathbf{h}^{\prime+}}, W,\left.V^{b}\right|_{\mathbf{h}^{\prime}}\right)$, our assumptions remain satisfied and the statement to be proved remains the same. Thus we are reduced to the case where $\mathbf{h}=\mathbf{h}^{\prime}$ and $\mathbf{h}^{+}=\mathbf{h}^{\prime+}$. In this case $W$ is an h-module.

Our assumption that $\left(\mathbf{h}, \mathbf{h}^{-}, \mathbf{h}^{+}, V\right)$ is a split induction datum implies that $\left(\mathbf{h}, \mathbf{h}^{-}, \mathbf{h}^{+},\left.V \otimes_{A} W\right|_{\mathbf{h}^{+}}\right)$is a split induction datum and that the corresponding h-module $\left(\left.V \otimes_{A} W\right|_{\mathbf{h}^{+}}\right)^{\mathrm{b}}$ is $V^{\mathrm{b}} \otimes_{A} W$.

Hence in the statement to be proved, $V$ and $W$ enter only together, through $V \otimes_{A} W$. Thus there is no loss of generality if we assume also that $W=A$ with the zero h-module structure. Therefore, our task is reduced to proving 
$\left.\left.V\right|_{\mathbf{h}^{+}} \cong V^{b}\right|_{\mathbf{h}}$. By the definition, we have $V^{b}=U(\mathbf{h}) \otimes_{U\left(\mathbf{h}^{+}\right)} V$ as an h-module. It follows that

$$
\left(V^{b}\right)_{\mathbf{h}}=A \otimes_{U(\mathbf{h})}\left(U(\mathbf{h}) \otimes_{U\left(\mathbf{h}^{+}\right)} V\right)=A \otimes_{U\left(\mathbf{h}^{+}\right)} V=V_{\mathbf{h}^{+}},
$$

as desired.

A.6. We will need a variant of the previous proposition. Let $h$ be an $A$-Lie algebra, and let $\mathbf{h}^{+}, \mathbf{h}^{\prime}, \mathbf{h}^{\prime+}$ be $A$-Lie subalgebras of $\mathbf{h}$ such that $\mathbf{h}=\mathbf{h}^{+}+\mathbf{h}^{\prime}$ and $\mathbf{h}^{\prime+}=\mathbf{h}^{+} \cap \mathbf{h}^{\prime}$. Let $V$ be a $\mathbf{h}^{+}$-module. We form the $\mathbf{h}$-module $\widetilde{V}$ induced by $V$ (see A.1). We restrict it to a $\mathbf{h}^{\prime}$-module and form $\widetilde{V}_{\mathbf{h}^{\prime}}$. We restrict $V$ to a $\mathbf{h}^{\prime+}$-module and form $V_{\mathbf{h}^{\prime+}}$.

The canonical homomorphism $V \rightarrow \widetilde{V}$ carries $\mathbf{h}^{\prime+} V$ to $\mathbf{h}^{\prime} \tilde{V}$; hence, we have an induced map

$$
V_{\mathbf{h}^{\prime+}} \rightarrow \widetilde{V}_{\mathbf{h}^{\prime}}
$$

In this setup, we have the following result.

Proposition A.7. (a) The linear map A.6(a) is surjective.

(b) Assume that there exist A-bases $\beta$ of $\mathbf{h}, \beta^{\prime}$ of $\mathbf{h}^{\prime}, \beta^{+}$of $\mathbf{h}^{+}$, and $\beta^{\prime+}$ of $\mathbf{h}^{\prime+}$ such that $\beta^{\prime+}=\beta^{\prime} \cap \beta^{+}$and $\beta^{\prime} \cup \beta^{+}=\beta$. Then the linear map A.6(a) is an isomorphism.

Since $\mathbf{h}=\mathbf{h}^{+}+\mathbf{h}^{\prime}$, multiplication in $U(\mathbf{h})$ defines a surjective $A$-linear map $U\left(\mathbf{h}^{\prime}\right) \otimes_{A} U\left(\mathbf{h}^{+}\right) \rightarrow U(\mathbf{h})$. It follows that $\widetilde{V}$ is generated as a $U\left(\mathbf{h}^{\prime}\right)$-module by the image of $V \rightarrow \widetilde{V}$. The surjectivity of A.6(a) follows.

We prove (b). In this case, we may use the Poincaré-Birkhoff-Witt theorem and we see that $U\left(\mathbf{h}^{\prime}\right) \otimes_{U\left(\mathbf{h}^{\prime+}\right)} U\left(\mathbf{h}^{+}\right) \cong U(\mathbf{h})$.

Hence $\widetilde{V}=U\left(\mathbf{h}^{\prime}\right) \otimes_{U\left(\mathbf{h}^{\prime+}\right)} V$. We have

$$
\begin{aligned}
\widetilde{V}_{\mathbf{h}^{\prime}} & =\left(U\left(\mathbf{h}^{\prime}\right) \otimes_{U\left(\mathbf{h}^{\prime+}\right)} V\right)_{\mathbf{h}^{\prime}} \\
& =\mathbf{C} \otimes_{U\left(\mathbf{h}^{\prime}\right)}\left(U\left(\mathbf{h}^{\prime}\right) \otimes_{U\left(\mathbf{h}^{\prime+}\right)} V\right) \\
& =\mathbf{C} \otimes_{U\left(\mathbf{h}^{\prime+}\right)} V=V_{\mathbf{h}^{\prime+}}
\end{aligned}
$$

The proposition is proved.

\section{REFERENCES}

[De] P. Deligne, Une description de catégorie tressée (inspiré par Drinfeld), unpublished.

[G] A. Grothendieck, Eléments de géométrie algébrique, IV (première partie), Inst. Hautes Études Sci. Publ. Math. 20 (1964).

[W] W. Wasow, Asymptotic expansions for ordinary differential equations, Interscience, New York, London, and Sydney, 1965.

Department of Mathematics, Harvard University, Cambridge, Massachusetts 02138 E-mail address: kazhdan@math.harvard.edu

Department of Mathematics, Massachusetts Institute of Technology, Cambridge, MASSACHUSETTS 02139

E-mail address: gyuri@math.mit.edu 